\title{
STABLE HIGH CONDUCTIVITY BILAYERED ELECTROLYTES FOR LOW TEMPERATURE SOLID OXIDE FUEL CELLS
}

\author{
FETC AAD Document Control Bldg. 921 \\ U.S. Department of Energy \\ Federal Energy Technology Center \\ P.O. Box 10940 \\ Pittsburgh, PA 15236-0940
}

Contract \# DE-AC26-99FT40712

Program Manager: Lane Wilson

Annual Report for Period 10/1/00 - 9/30/01

Eric D. Wachsman and Keith L. Duncan

Department of Materials Science and Engineering

University of Florida

Gainesville, FL 32611-6400 


\section{CONTENTS}

EXECUTIVE SUMMARY ......................................................................... iii

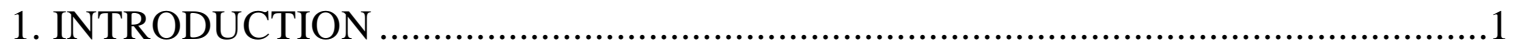

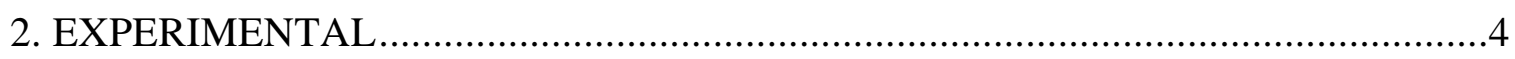

2.1 Fabrication and Characterization............................................................4

2.1.1 Samaria-doped ceria (SDC) .....................................................

2.1.2 Erbia-stabilized bismuth oxide (ESB) ..............................................6

2.1.3 A new oxygen ion conductor: dysprosium and tungsten stabilized bismuth oxide (DWSB) .........................................................6

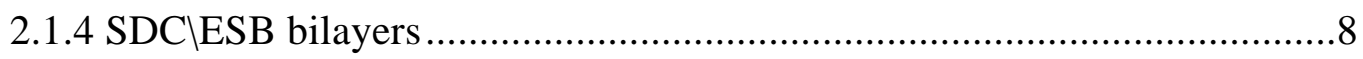

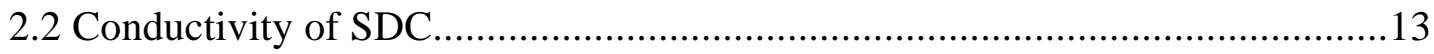

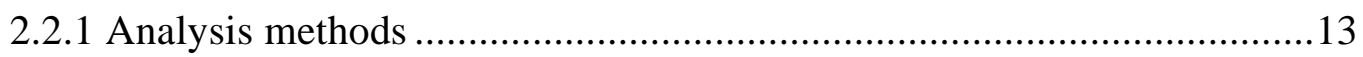

2.2.2 Effect of processing parameters on SDC..............................................13

2.2.3 Effect of grain boundary impurities in SDC ........................................16

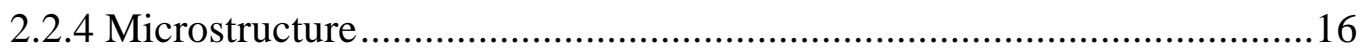

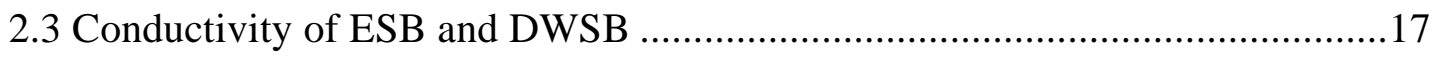

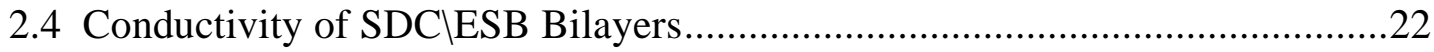

2.5 Fabrication of Anode-Supported Ceria Electrolytes by Colloidal Deposition.....24

3. ANALYTICAL MODEL FOR DEFECT DISTRIBUTION AND TRANSPORT

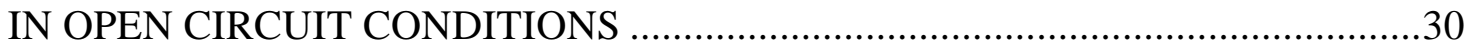

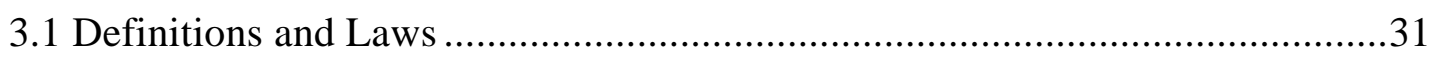

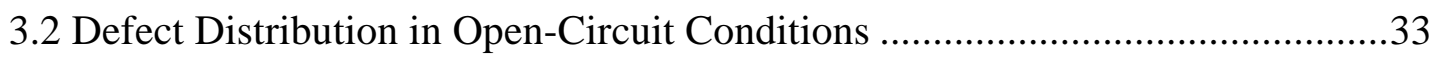

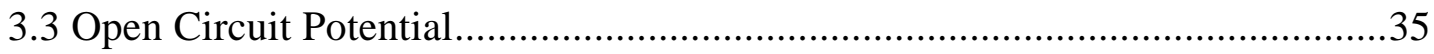

3.4 Evaluating the Magnitude of the External Equilibrium Constant, $K_{r} \ldots \ldots \ldots \ldots \ldots . . . .36$

3.4.1 Estimation of $K_{r}$ from thermogravimetry ..........................................36

3.4.2 Estimation of $K_{r}$ from open-circuit potential ..................................... 37

3.4.3 Estimation of $K_{r}$ from conductivity measurements ..............................40

4. OPEN CIRCUIT POTENTIAL AND MODEL VERIFICATION FOR SDC

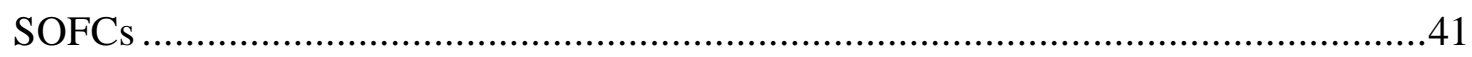

4.1 Open Circuit Potential Measurement.............................................................41

4.2 Effect of Cathode Material (Au and Pt) ..................................................45

4.3 Experimental Verification of the Model .......................................................50

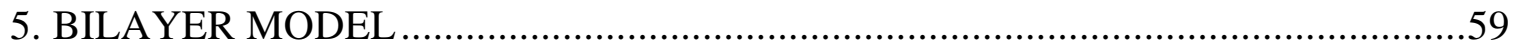


5.1 Open-Circuit Conditions and Thickness Ratio................................................59

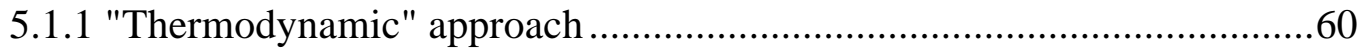

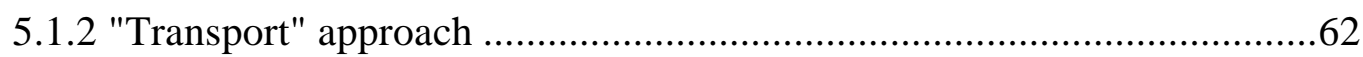

5.1.3 Results and discussion................................................................63

5.2 Closed-Circuit Conditions and Thickness Ratio ..........................................66

5.2.1 Model....................................................................................66

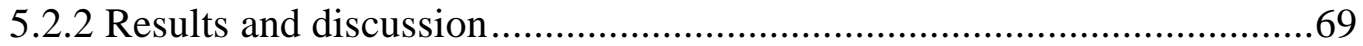

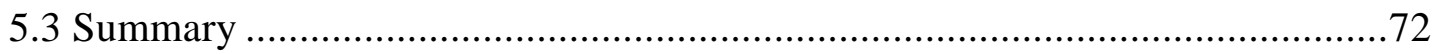

6. EXPERIMENTAL RESULTS FOR SDCIESB BILAYER SOFCs ..........................72

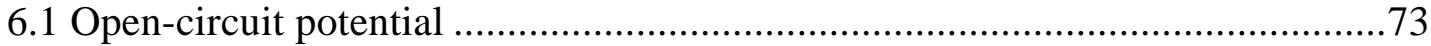

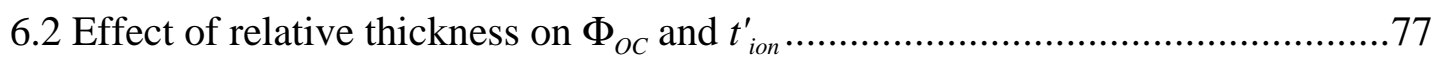

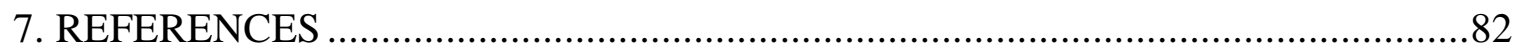




\section{EXECUTIVE SUMMARY}

In this annual report to the US Department of Energy, we summarize the results of our research on stable high conductivity bilayered electrolytes for low temperature solid oxide fuel cells (Contract \# DE-AC26-99FT40712). The report covers our progress in experimentation and computer modeling, and includes experimental verification of the model.

Initial experimental work focused on optimizing the fabrication of samaria-doped ceria (SDC) in order to maximize its density and conductivity. This is important because of SDCs notoriously poor sinterability. We then deposited an erbia stabilized bismuth oxide (ESB) layer on the SDC substrates using pulsed laser deposition (PLD). Because of the time needed for deposition, we were able to deposit only very thin $(<2 \mu \mathrm{m})$ ESB layers. Evaluation of the microstructure revealed that the ESB film adhered well to the SDC substrate and interdiffusion was not detectable. Further, we found that the resistance of the bilayer was in fact less than that of the SDC alone, confirming at the very least that no lower conducting phase formed at the bilayer interface.

We have also developed a new oxygen ion conductor based on $\mathrm{Bi}_{2} \mathrm{O}_{3}$ doped with $\mathrm{Dy}_{2} \mathrm{O}_{3}$ and $\mathrm{WO}_{3}$ (DyWSB). It exhibits a higher conductivity than that of $20 \mathrm{~mol} \%$ erbia stabilized bismuth oxide (20ESB), thus, giving it the highest conductivity of any known solid oxide electrolyte. Electrical conductivity results of the Dy - W stabilized bismuth oxide system are presented. The dopants were selected based on their polarizability and its effect on structural stability and conductivity. At $800^{\circ} \mathrm{C}$ the conductivity of $\left(\mathrm{BiO}_{1.5}\right)_{0.88}\left(\mathrm{DyO}_{1.5}\right)_{0.08}\left(\mathrm{WO}_{3}\right)_{0.04}$ is 0.57 $\mathrm{S} / \mathrm{cm}(1.5$ times as high as that of $20 \mathrm{ESB})$, and at $500^{\circ} \mathrm{C}$ the conductivity is $0.043 \mathrm{~S} / \mathrm{cm}$ (2 times as high as that of 20ESB). Consequently, because its conductivity is even higher than ESB, we anticipate even better performance than originally predicted when it is incorporated into the bilayer.

We examined the electrochemical performance of SDC and ESB/SDC bilayer SOFCs as a function of anode $\mathrm{P}_{\mathrm{O}_{2}}$ under open-circuit conditions (sections 4 and 6). SDC was examined first (section 4) to establish a suitable baseline for comparison. Our SDC based concentration cells performed as expected, with a low open-circuit potential (OCP) and effective ionic transference number $\left(t_{i o n}\right)$ due to electronic conduction. In contrast, results for SOFCs with bilayered electrolytes (section 6) showed they had higher OCP and $t_{i o n}$ values (approaching unity) compared to those with a single SDC layer. Further, we confirmed that OCP and $t_{i o n}$ increased as the relative thickness of the ESB layer increased, thereby re-confirming the bilayer concept. Despite this achievement, however, there is still work to be done on optimizing the thickness 
ratio of the constituent layers (to achieve maximum performance) and evaluating the closed circuit performance of the bilayer.

We have also begun work on developing suitable anodes for the bilayers and a technique for depositing acceptor (samaria or gadolinia) doped ceria on them to form the basis for an anode supported bilayered SOFC. Investigation into the effect of relative thickness of the bilayers is further facilitated by electrode supported electrolytes, as fabrication of the bilayer requires some ingenuity due to the large difference in the sintering temperatures of bismuth oxide and ceria. As a first step, we report the fabrication and performance of Ni-gadolinium doped ceria (GDC) anode supported GDC thick film electrolyte, using a colloidal deposition method. Colloidal deposition has advantages over other techniques for thick film processing in its simplicity, costeffectiveness, upscalibility and flexibility (thickness ranging from 10-100 $\mu \mathrm{m}$ can be deposited). Further, under the reducing conditions at the anode doped ceria is a mixed ionic electronic conductor and hence, the reaction zone will not be restricted to the interface of the anode and the electrolyte leading to enhanced performance of the anode.

In order to further establish a sound theoretical basis for our approach, we have also extended, revised and applied our theoretical models for the generation, distribution and transport of defect in oxide mixed ionic-electronic conductors. For the sake of brevity, the report omits much of the model development and also focuses primarily (though not exclusively) on open-circuit conditions. In section 3, a model for defect distribution is developed based on the usual transport laws for charged species in a concentration gradient. The model is used (along with earlier models) to show how essential material constants may be obtained from various experimental data. In section 4 , the model was verified through fitting to experimental data (measured vs. theoretical OCP). In all cases, correlation coefficients (corrected for two degrees of freedom) greater than $95 \%$ were obtained, thus, confirming the validity of the model.

Finally, the bilayer model, presented in an earlier report, was revised and extended to closed circuit conditions. In the revision, two very disparate approaches were taken and shown to yield essentially identical results for open-circuit conditions, thereby corroborating the validity of each. Perhaps of more interest, however, is that the predictions show that low area specific resistances (ASRs) are achievable using bilayer architecture for fuel cell electrolytes in opencircuit conditions.

Even more appealing results were obtained when the bilayer was modeled for closedcircuit conditions. In closed-circuit conditions, electronic species will flow primarily through the external circuit especially in the presence of the electron-blocking ESB layer. Consequently, the sole diffusing species inside the electrolyte (in closed-circuit conditions) are oxygen vacancies 
and ESB is much less resistive to their transport than SDC. Therefore, the potential drop per unit length is much larger for SDC than ESB and a thin SDC layer will sustain enough voltage to protect the sensitive ESB layer. This situation is in contrast to open-circuit conditions where the diffusing species (oxygen atoms via ambipolar diffusion) is electrically neutral and ESB is the more resistive layer due to the unavailability (in comparison to SDC) of electronic species.

In closing, both experiment and theory support the viability of stable high conductivity bilayered electrolytes for low temperature solid oxide fuel cells. More work remains to be done, but present results demonstrate that we are on the right course. 


\section{INTRODUCTION}

Solid oxide fuel cells (SOFCs) are the future of energy production in America. They offer great promise as a clean and efficient process for directly converting chemical energy to electricity while providing significant environmental benefits (they produce negligible hydrocarbons, $\mathrm{CO}$, or $\mathrm{NO}_{\mathrm{x}}$ and, as a result of their high efficiency, produce about one-third less $\mathrm{CO}_{2}$ per kilowatt hour than internal combustion engines).

Unfortunately, the current SOFC technology, based on a stabilized zirconia electrolyte, must operate in the region of $1000^{\circ} \mathrm{C}$ to avoid unacceptably high ohmic losses. These high temperatures demand (a) specialized (expensive) materials for the fuel cell interconnects and insulation, (b) time to heat up to the operating temperature and (c) energy input to arrive at the operating temperature. Therefore, if fuel cells could be designed to give a reasonable power output at low to intermediate ${ }^{1}$ temperatures tremendous benefits may be accrued. At low temperatures, in particular, it becomes feasible to use ferritic steel for interconnects instead of expensive and brittle ceramic materials such as those based on $\mathrm{LaCrO}_{3}$. In addition, sealing the fuel cell becomes easier and more reliable; rapid start-up is facilitated; thermal stresses (e.g., those caused by thermal expansion mismatches) are reduced; radiative losses $\left(\sim \mathrm{T}^{4}\right)$ become minimal; electrode sintering becomes negligible and (due to a smaller thermodynamic penalty) the SOFC operating cycle (heating from ambient) would be more efficient. Combined, all these improvements further result in reduced initial and operating costs. The problem is, at lower temperatures the conductivity of the conventional stabilized zirconia electrolyte decreases to the point where it cannot supply electrical current efficiently to an external load.

The primary objectives of the proposed research are to develop a stable high conductivity (> $0.05 \mathrm{~S} \mathrm{~cm}^{-1}$ at $\leq 550{ }^{\circ} \mathrm{C}$ ) electrolyte for lower temperature SOFCs. This objective is specifically directed toward meeting the lowest (and most difficult) temperature criteria for the $21^{\text {st }}$ Century Fuel Cell Program. Meeting this objective provides a potential for future transportation applications of SOFCs, where their ability to directly use hydrocarbon fuels could permit refueling within the existing transportation infrastructure.

In order to meet this objective we are developing a functionally gradient bilayer electrolyte comprised of bismuth oxide on the air side and ceria on the fuel side. Bismuth oxide and doped ceria are among the highest ionic conducting electrolytes and in fact bismuth oxide

\footnotetext{
${ }^{1}$ In accordance with current convention, the term "low" is used here to for temperatures $<600{ }^{\circ} \mathrm{C}$ while the term "intermediate" refers to temperatures in the range $500-800^{\circ} \mathrm{C}$.
} 
based electrolytes are the only known solid oxide electrolytes to have an ionic conductivity that meets the program conductivity goal.

The bilayered electrolyte we are developing consists of a layer of erbia-stabilized bismuth oxide (ESB) on the oxidizing side and a layer of SDC or GDC on the reducing side. In this arrangement, the ceria layer protects the bismuth oxide layer from decomposing by shielding it from very low $P_{\mathrm{O}_{2}}$ 's and the ESB layer serves to block electronic flux through the electrolyte. This arrangement has two significant advantages over the YSZISDC bilayers investigated by others $[1,2]$. The first advantage is that SDC is conductive enough to serve as an intermediate temperature SOFC electrolyte. Moreover, ESB is conductive enough to serve as a low temperature electrolyte. Consequently, at worst an SDC\ESB bilayered SOFC should have the conductivity of SDC but with improved efficiency due to the electronic flux barrier provided by ESB. The second advantage is that small (dopant) concentrations of SDC in ESB or ESB in SDC, have been found to have conductivities comparable to the host lattice $[3,4]$. Therefore, if solid solutioning occurs at the SDC-ESB interface, it should not be detrimental to the performance of the bilayer. In contrast, solid solutions of SDC and YSZ have been found to be significantly less conductive than SDC or YSZ. Thus, it bears emphasizing that, at this time, only SDCIESB electrolytes have potential in low temperature SOFC applications.

The primary issue concerning SDC $\ E S B$ bilayers, is assessing the stability of ESB in this arrangement for various fuel cell operating conditions and optimizing the relative thickness of the SDC and ESB layers so that the oxygen partial pressure at the SDC-ESB interface is high enough to prevent decomposition of the ESB. In open-circuit conditions the stability of ESB must be determined by calculating (since direct measurement is not presently feasible) the effective $P_{\mathrm{O}_{2}}$ at the interface, which, as shown in Figure 1-1, is a function of the relative thicknesses of the layers. Ideally, the effective interfacial $P_{\mathrm{O}_{2}}$ should not fall below $\sim 10^{-13}$ atm. (i.e., the $P_{\mathrm{O}_{2}}$ bismuth oxide below which decomposes) for the SDCLESB bilayer. Under closedcircuit conditions one can no longer talk about an effective interfacial $P_{\mathrm{O}_{2}}$ since the oxygen is no longer permeating by ambipolar diffusion through the bilayer. Therefore, as shown later, other indicators of stability must be used.

We have previously demonstrated that this concept works, that a bismuth oxide/ceria bilayer electrolyte provides near theoretical open circuit potential (OCP) and is stable for $1400 \mathrm{~h}$ of fuel cell operation under both open circuit and maximum power conditions. More recently, we developed an analytical model to determine the defect transport in this bilayer and have found that a bilayer comprised primarily of the more conductive component (bismuth oxide) is stable for $500^{\circ} \mathrm{C}$ operation. 

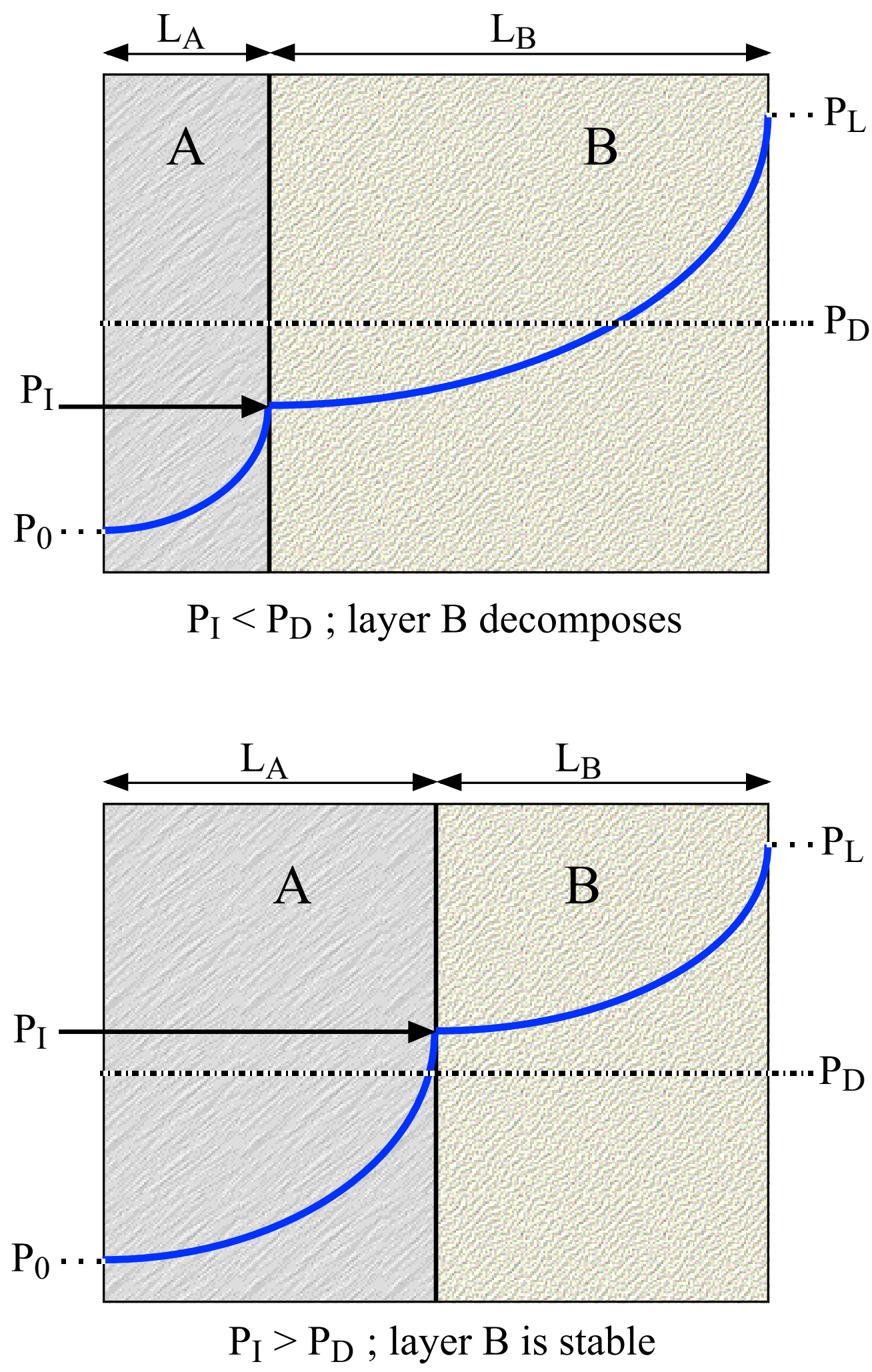

Figure 1-1. Conceptual representation of a bilayered electrolyte showing the effect of relative thickness on interfacial $P_{\mathrm{O}_{2}}, \mathrm{P}_{\mathrm{I}} ; \mathrm{P}_{0}$ is the $P_{\mathrm{O}_{2}}($ at $\mathrm{x}=0)$ on the fuel/anode side, $\mathrm{P}_{\mathrm{L}}$ is the $P_{\mathrm{O}_{2}}($ at $\mathrm{x}=$ L) on the air/cathode side and $\mathrm{P}_{\mathrm{D}}$ is the decomposition $P_{\mathrm{O}_{2}}$. 
In this report, the fabrication and characterization of single-layered electrolytes of SDC, ESB and bilayered electrolytes of SDCLESB are presented. Fabrication techniques include standard ceramic processing with uni-axial pressing and with cold isostatic pressing (CIP). Characterization techniques include $\mathrm{x}$-ray diffraction, density analysis, scanning electron microscopy (SEM), x-ray photo-electronic spectroscopy (XPS), energy dispersive spectroscopy (EDS) and electrical impedance spectroscopy (EIS) and open-circuit potential (OCP) measurements. The report also contains a (revised) model that was developed to predict the spatial distribution and transport properties of ionic and mixed ionic-electronic conductors (MIECs). First, the correctness of the model is verified by fitting it to experimental data for the OCP of an SDC based SOFC. Then the model is applied to bilayered electrolytes in order to obtain a relationship between the thickness ratio of the constituent layers of the SDC\ESB bilayer and interfacial conditions-i.e., an oxygen potential, $P_{\mathrm{O}_{2}}$, drop (in open circuit conditions) or an electrical potential drop (in closed-circuit conditions) — for various fuel cell operating conditions. Finally, the model's predictions are again compared to experimental results for the OCP of SDCLESB bilayered SOFCs.

\section{EXPERIMENTAL}

Previously, we completed the assembly, calibration and testing of the SOFC apparatus and a preliminary sintering study. Since then, the sintering study (of particular interest to SDC fabrication because of its extremely high sintering temperature) has been completed and is

presented in this section. SDC and SDCLESB samples were also analyzed to ensure that they had the correct (fluorite) structure, (which also served as an indirect test for the presence of secondary impurity phases) and the correct chemical composition and a suitable (and acceptably pore-free) microstructure. Finally, SDC and SDCLESB samples were characterized for electrical conductivity via electrical impedance spectroscopy (EIS) and, for the former, the impact of processing techniques, impurities and microstructure on conductivity was assessed.

\subsection{FABRICATION AND CHARACTERIZATION OF SDC AND SDC\ESB BILAYER}

\subsubsection{Samaria-Doped Ceria (SDC)}

SDC powders were calcined at 1100,1200 and $1300^{\circ} \mathrm{C}$ for $10 \mathrm{hrs}$ and sintered at $1465^{\circ} \mathrm{C}$, $1550{ }^{\circ} \mathrm{C}$, and $1650{ }^{\circ} \mathrm{C}$ for $15 \mathrm{hrs}$. The densities of the SDC pellets prepared were found to be $70-98 \%$ of the theoretical density depending on the sintering temperature. Figure 2-1 shows the measured relative densities of SDC samples as a function of axially applied pressure by changing 


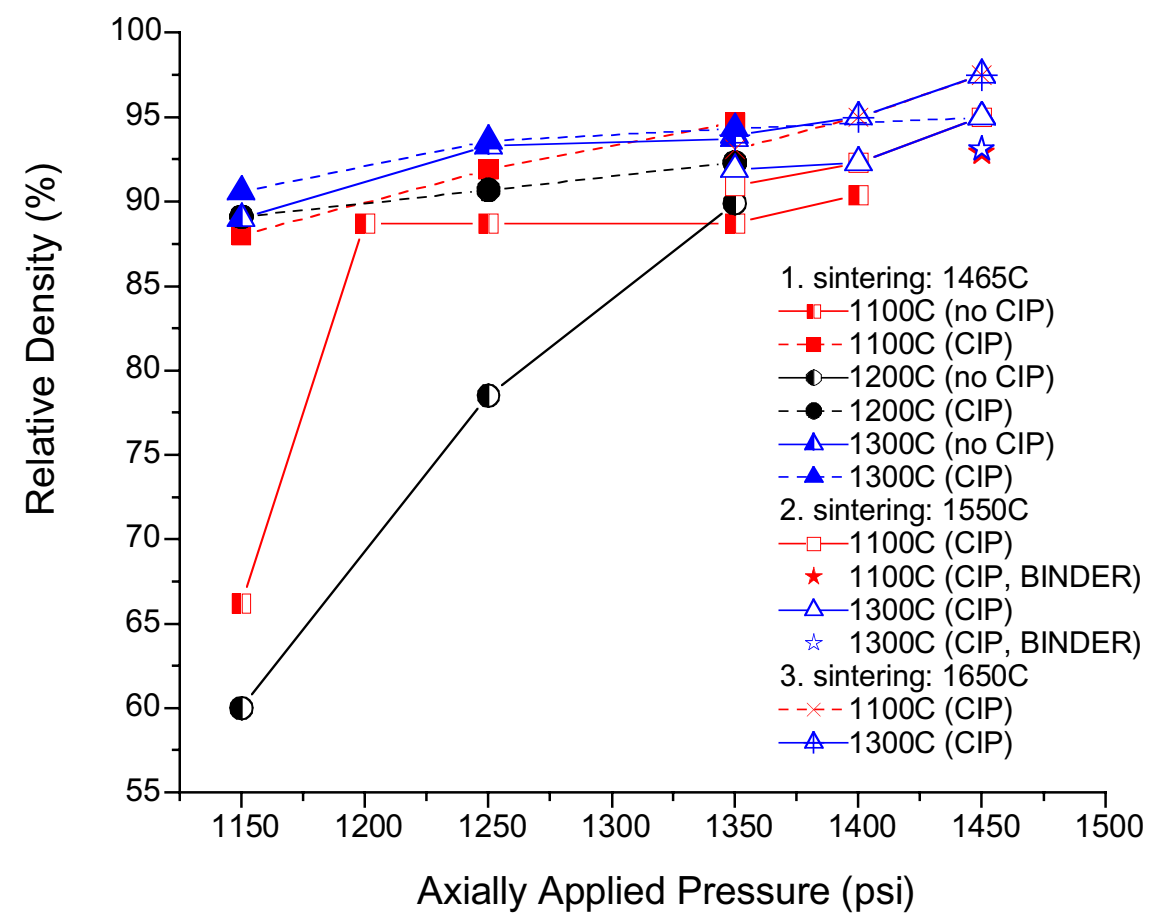

Figure 2-1. Effect of axially and isostatically pressing, calcining and sintering temperature, and binder (P.E.G. 8000) on SDC pellets

sintering temperature, calcination temperature, binder, and the use of cold-isostatic pressing (CIP). The density, $\rho$, of the sintered samples increased with the sintering temperature. Fabrication of SDC pellets by the solid-state technique required a fairly high temperature of 1600 ${ }^{\circ} \mathrm{C}$ in order to get more than $95 \%$ theoretical density. However, sintering at these temperatures is expensive and makes co-firing doped ceria with other materials difficult due to severe solid-state reaction and inter-diffusion at those temperatures [5]. Thus, it would be beneficial to fabricate doped ceria electrolyte at the lowest temperature as possible. To this end, Huang et al. [6] demonstrated that the small average particle size of sol-gel prepared green-bodies allows sintering of SDC samples into highly dense pellets at a significantly lower temperature of $1400^{\circ} \mathrm{C}$, versus $1600^{\circ} \mathrm{C}$ required for conventionally prepared samples.

Theoretically, denser and/or stronger green-bodies may be obtained through one or a combination of: increased pressing load, binder (typical organic liquids) reinforcement or isostatic pressing. All three methods were tested in an attempt to simultaneously increase the density and lower the sintering temperature of SDC samples.

The uni-axial pressing load was increased systematically for SDC samples, Figure 2-1. However, it was found that increasing the uni-axial pressing load above 1500 psi causes delamination and end capping - two of the more common defects in die-compacted powders [7]. 
The binder polyethylene glycol (PEG) 8000 was tried in an attempt to increase the greenbody strength. However, it was found (rather unexpectedly) that after sintering, the densities of the samples prepared with the PEG binder were lower than those prepared without it. This result was surprising because the PEG binder had increased the observed strength of the green-body after compaction (pressing). The reduction in sintered sample density may be attributable to structural anisotropy introduced by the binder that resulted in voids formation during sintering.

The highest SDC sample densities were achieved with cold-isostatically pressed samples. Cold-isostatic pressing increased the densities of the sintered samples because the pressing load is uniform and, consequently, powder movement (which causes de-lamination) is reduced [7].

\subsubsection{Erbia-Stabilized Bismuth Oxide (ESB)}

Disks of 20 mol.\% $\mathrm{Er}_{2} \mathrm{O}_{3}-\mathrm{Bi}_{2} \mathrm{O}_{3}$ were fabricated by a solid state reaction. $\mathrm{Bi}_{2} \mathrm{O}_{3}$ (99.9995\% pure) and $\mathrm{Er}_{2} \mathrm{O}_{3}(99.99 \%$ pure) powders were obtained from Alfa Aesar. The powders were mixed and ball-milled with magnesia stabilized zirconia ball media in a Nalgene ${ }^{\circledR}$ jar for 20 hours. The mixed powders were calcined at $800^{\circ} \mathrm{C}$ for 16 hours. Agglomerated powders were ground again using mortar and pestle and pressed into a disk-shaped 1 1/8-inch die using 5000 psi for OCP measurements and 1/4 inch die using 3000 psi for conductivity measurements. Polyvinyl alcohol (PVA) and stearic acid mixed with reagent alcohol were added as a binder and a lubricant respectively. The resulting green bodies were sintered in air at $890^{\circ} \mathrm{C}$ for 16 hours. After sintering, the specimens were polished to acquire even surfaces and desired thicknesses (1.5 $\mathrm{mm}$ and $2 \mathrm{~mm}$ for OCP and conductivity experiments respectively).

\subsubsection{A New Oxygen Ion Conductor: Dy and W Stabilized Bismuth Oxide (DWSB)}

The high ionic conductivity of $\delta-\mathrm{Bi}_{2} \mathrm{O}_{3}$ is ascribed both to the large number of oxygen vacancies and a high anion mobility. The high concentration of oxygen vacancies $(25 \%$ of the lattice sites) is a result of obtaining the fluorite structure with a $\mathrm{Bi}^{3+}$ cation. The high anion mobility is related to the high polarizability of the $\mathrm{Bi}^{3+}$ cation with its lone pair of electrons [8].

Pure bismuth oxide transforms to monoclinic $\alpha$-phase on cooling below $730^{\circ} \mathrm{C}$. The cubic phase at high temperature can be stabilized down to room temperature by the additions of dopants such as isovalent rare earth cations. However, these doped bismuth oxides show lower conductivity and undergo an order-disorder transition of the oxygen sublattice below $600^{\circ} \mathrm{C}$, which leads to a decay in conductivity (aging effect) [9 - 11]. Our results have shown that the dopant cations affect the nature of the vacancy arrangement in the oxygen sublattice $[9,12,13]$ and the polarizability of the cation affects the stability of the disordered oxygen sublattice [14]. 
These results showed that the most polarizable of the lanthanide dopants, Dy, provided the greatest stability for the disordered anion lattice [14]. Among the potential dopants to stabilize the fluorite structure of $\mathrm{Bi}_{2} \mathrm{O}_{3}$, W has the highest polarizability and therefore should also provide stability for the disordered anion lattice. Moreover, based on the results of Battle et al [8], the high polarizability of Dy and $\mathrm{W}$ should result in a high anion mobility.

Takahashi et al [15] reported that $\mathrm{WO}_{3}$ stabilized $\mathrm{Bi}_{2} \mathrm{O}_{3}$ has high conductivity, but the fcc phase was stable only over a narrow composition range (22 - $28 \mathrm{~mol} \% \mathrm{WO}_{3}$ in $\mathrm{Bi}_{2} \mathrm{O}_{3}$ ). Verkerk and Burggraaf [16] investigated the phase stability and oxygen ion conductivity of $\mathrm{Dy}_{2} \mathrm{O}_{3}$ stabilized $\mathrm{Bi}_{2} \mathrm{O}_{3}$. They found that the cubic structure was stable at low temperatures for the solid solutions containing $28.5-50 \% \mathrm{Dy}_{2} \mathrm{O}_{3}$. In both investigations it was found that the highest conductivity was obtained for the lowest dopant concentration that would stabilize the fcc phase $\left(\left(\mathrm{BiO}_{1.5}\right)_{0.88}\left(\mathrm{WO}_{3}\right)_{0.12}\right.$ and $\left.\left(\mathrm{BiO}_{1.5}\right)_{0.715}\left(\mathrm{DyO}_{1.5}\right)_{0.285}\right)$. Therefore, based on the cation ratio the fcc structure can be stabilized with $\mathrm{W}$ at a lower dopant concentration than with Dy. In addition, studies $[17,18]$ have shown that the face-centered cubic structure in the $\mathrm{Bi}_{2} \mathrm{O}_{3}$-based solid solutions can be stabilized down to room temperature with two rare earth oxide dopants with smaller total dopant concentrations. Since smaller dopant concentrations have higher conductivity, this results in an increase in conductivity, especially in the lower temperature regions. Therefore, we tried doping $\mathrm{Bi}_{2} \mathrm{O}_{3}$ with both $\mathrm{Dy}_{2} \mathrm{O}_{3}$ and $\mathrm{WO}_{3}$ (DyWSB), the highly polarizable dopants, in order to obtain a structurally stable electrolyte with high conductivity.

Disks of $\mathrm{Dy}_{2} \mathrm{O}_{3}-\mathrm{WO}_{3}-\mathrm{Bi}_{2} \mathrm{O}_{3}$ were fabricated for conductivity measurements by solid state reaction. Disks of $\left(\mathrm{BiO}_{1.5}\right)_{0.8}\left(\mathrm{ErO}_{1.5}\right)_{0.2}, 20 \mathrm{ESB}$, were also prepared for comparison of conductivity results. Six compositions were investigated: $\left(\mathrm{BiO}_{1.5}\right)_{0.87}\left(\mathrm{DyO}_{1.5}\right)_{0.10}\left(\mathrm{WO}_{3}\right)_{0.03}$, $\left(\mathrm{BiO}_{1.5}\right)_{0.88}\left(\mathrm{DyO}_{1.5}\right)_{0.08}\left(\mathrm{WO}_{3}\right)_{0.04},\left(\mathrm{BiO}_{1.5}\right)_{0.89}\left(\mathrm{DyO}_{1.5}\right)_{0.06}\left(\mathrm{WO}_{3}\right)_{0.05}$, and $\left(\mathrm{BiO}_{1.5}\right)_{0.83}\left(\mathrm{DyO}_{1.5}\right)_{0.17-\mathrm{x}}\left(\mathrm{WO}_{3}\right)_{\mathrm{x}}$ (where $\mathrm{x}=0.05,0.08,0.12$ ). The latter 3 compositions with a fixed $17 \%$ total dopant content are 12Dy5WSB, 9Dy8WSB and 5Dy12WSB. The former 3 compositions with a total dopant content between 11-13\% are 10Dy3WSB, 8Dy4WSB and 6Dy5WSB.

Powders of $\mathrm{Bi}_{2} \mathrm{O}_{3}$ (99.9995\% pure), $\mathrm{Er}_{2} \mathrm{O}_{3}$ (99.99\% pure), $\mathrm{Dy}_{2} \mathrm{O}_{3}\left(99.99 \%\right.$ pure) and $\mathrm{WO}_{3}$ (99.8\% pure), from Alfa Aesar, were mixed and ball-milled with zirconia ball media in a highdensity polyethylene bottle for 20 hours. The mixed powders were calcined at $800^{\circ} \mathrm{C}$ for 16 hours. Agglomerated powders were ground again using mortar and pestle and pressed into a disk-shaped $0.635 \mathrm{~cm}$ die using $2 \times 10^{5} \mathrm{~N} / \mathrm{m}^{2}$. Poly vinyl alcohol was used as a binder and stearic acid mixed with reagent alcohol as a lubricant. The subsequent green bodies were sintered in air at $890^{\circ} \mathrm{C}$ for 16 hours. Powder X-ray diffraction (XRD) data were obtained at room temperature for phase analysis. The XRD patterns were obtained using $\mathrm{CuK} \alpha$ radiation with a nickel filter. All compositions were found to be fcc structure. 
After sintering, the specimens were polished to acquire an even surface and the desired thickness $(2 \mathrm{~mm})$. Engelhard gold paste mixed with isopropanol was brushed onto both sides of the electrolytes, and the organic additives were evaporated at $127^{\circ} \mathrm{C}$ for 30 minutes. Subsequently the electrodes were sintered at $800^{\circ} \mathrm{C}$ for 30 minutes. Gold wires $(99.95 \%$ pure, DFG) spot-welded with circular gold mesh (99.9\% pure, 100 mesh, DFG) were attached to the cells using Au paste and were sintered again at $800^{\circ} \mathrm{C}$ for 30 minutes.

\subsubsection{SDC\ESB Bilayers}

Sintered SDC pellets were coated on one face with a thin film of ESB via pulsed laser deposition (PLD) using a Lambda Physik pulsed excimer laser. For coating, an ESB target was rotated at $2 \mathrm{RPM}$, the laser was pulsed at $5 \mathrm{~Hz}$ at a laser energy of $400 \mathrm{~mJ}$, with a substrate temperature of $700^{\circ} \mathrm{C}$. Film thickness was measured by elipsometry of films deposited on $\mathrm{Si}$ with identical deposition parameters.

Figure 2-2 shows a linear relationship between film thickness and deposition time for the growth of an ESB film on a SDC substrate. A key feature of the ESB film is that it should be pinhole free to block access of the cathode (or cathodic conditions) to SDC. By so doing, the ESB layer prevents electronic conduction (which occurs in single layer SDC membranes) in the bilayered membrane. In the present study, the thickness of the ESB film was limited to $2 \mu \mathrm{m}$. This limitation was imposed by the long depositions times required (it took several hours each to evacuate, heat up, deposit and cool down).

The desired structure for SDC and ESB is the fluorite structure. The XRD patterns in Figure 2-3 show that a fluorite structure was obtained for SDC, ESB and the bilayered ESB $\backslash$ SDC samples. Although expected, this result also indicates that other phases (arising from reactions between SDC and ESB at their interface) are not present to any large degree and, by extension, that PLD is an acceptable way to deposit ESB on an SDC substrate.

Figure 2-4 shows an SEM micrograph of the cross-section of an ESB film (deposited by PLD) on a dense SDC substrate (i.e., the bilayered ESB $\backslash$ SDC electrolytes). Cross-sectional views indicate that the film adhered well to the baseline ceria substrate. The chemical composition of the bilayered ESB $\backslash$ SDC electrolytes was obtained, Figure 2-5, from energy dispersive analysis (EDS). The SEM in Figure 2-5(a) shows the region that was line-scanned and Figures 2-5(b) (graph) and 2-5(c) (picture) show the compositional results of the linescan. It is apparent (at this resolution) in Figure 2-5(b), that neither solid-state reaction nor interdiffusion between the phases has occurred. Finally, the EDS spectra, in Figure 2-5(d), of the 
SDC \ESB sample cross-section in Figure 2-5(a) shows that any impurity levels are below detectable limits.

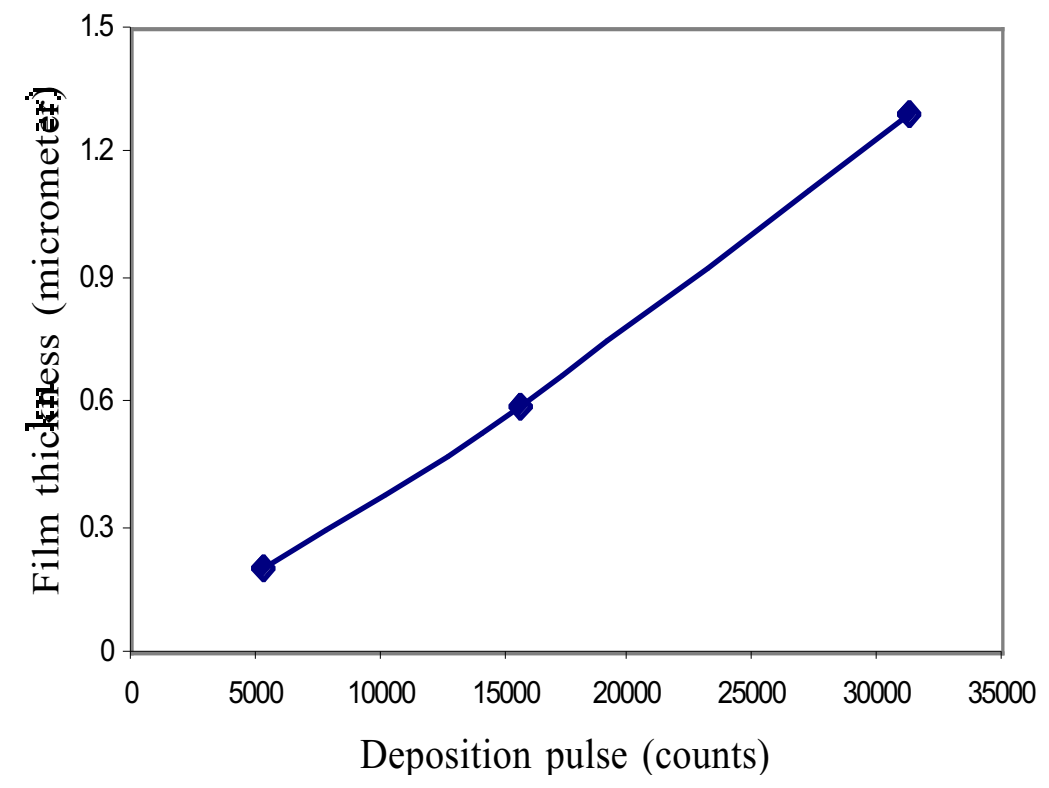

Figure 2-2. Thin film thickness on SDC substrate as a function of deposition time (pulses) 


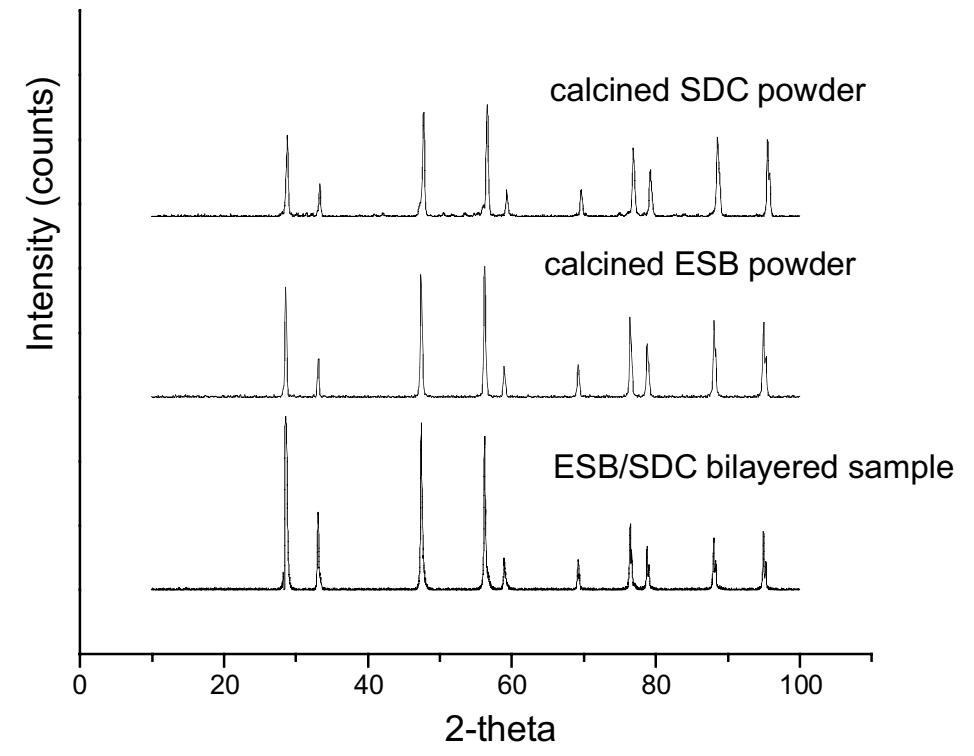

Figure 2-3. X-ray diffraction patterns of (top) SDC powder (middle) ESB powder and (bottom) SDC coated with ESB (bilayered SDCLESB electrolyte, film thickness: $0.2 \mu \mathrm{m}$ ) via PLD.

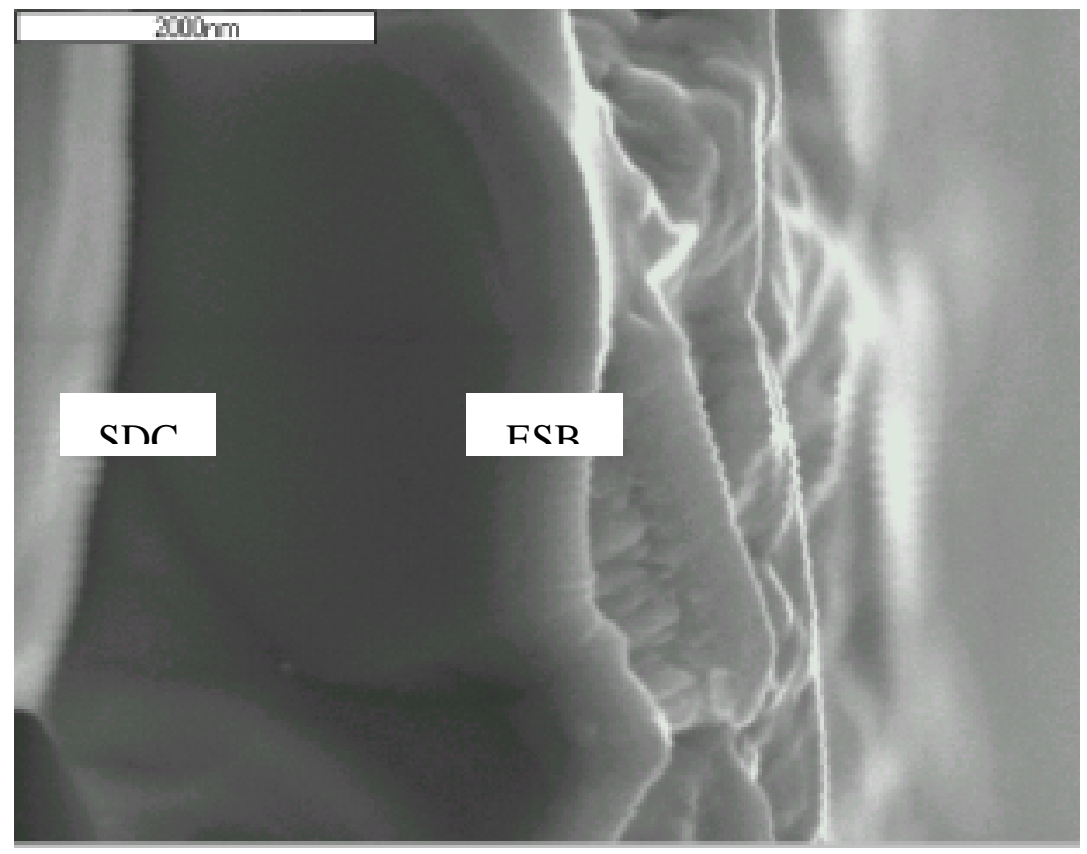

Figure 2-4 Cross-section SEM of ESB film on a dense SDC substrate (ESBISDC bilayer) 


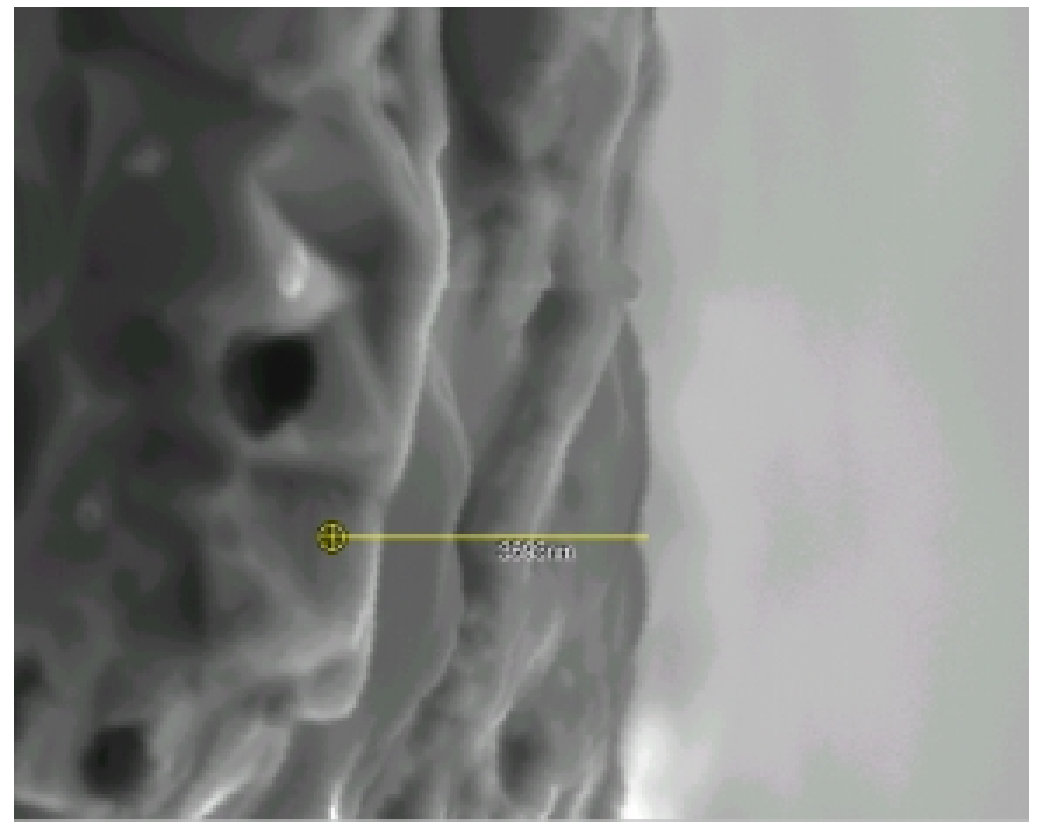

Figure 2-5(a). Line scanned SEM image of SDCIESB sample.

IHAGE, 1.135724E+07

BSE, 4999180

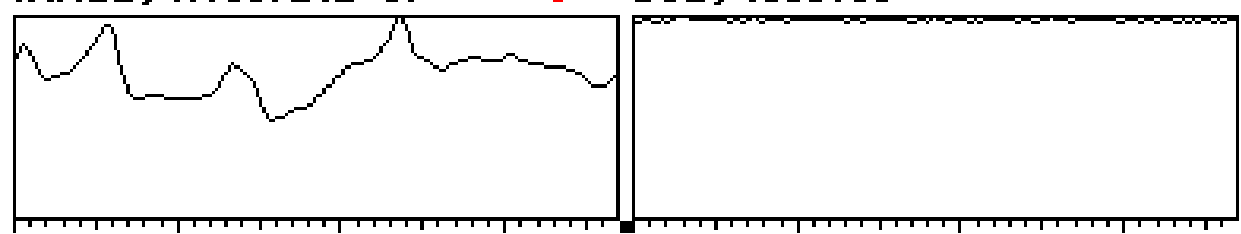

EriHa, 87

Bila1, 207
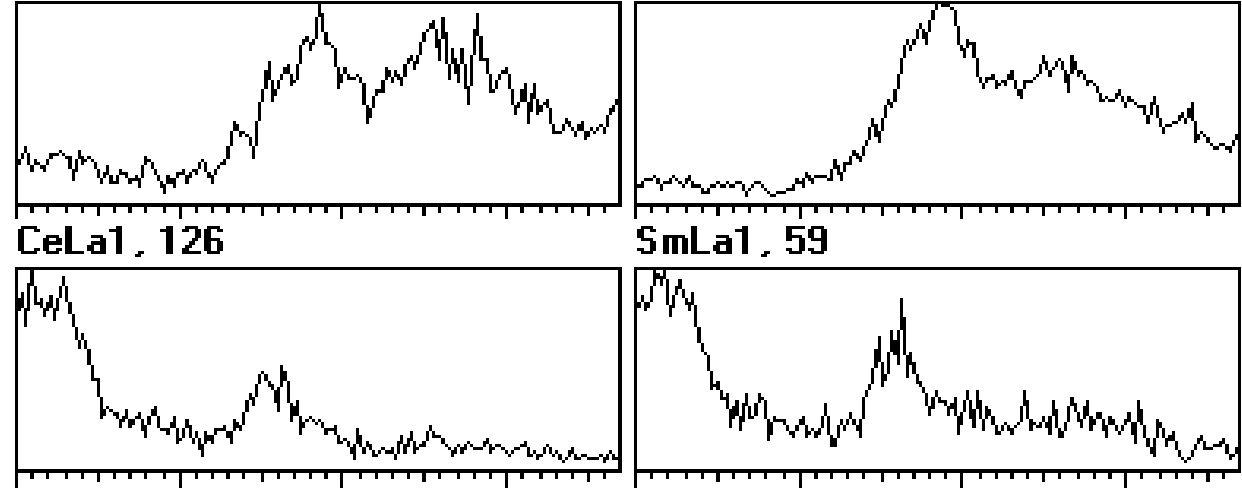

Figure 2-5(b). X-ray line-scan of elements of SDC\ESB sample 

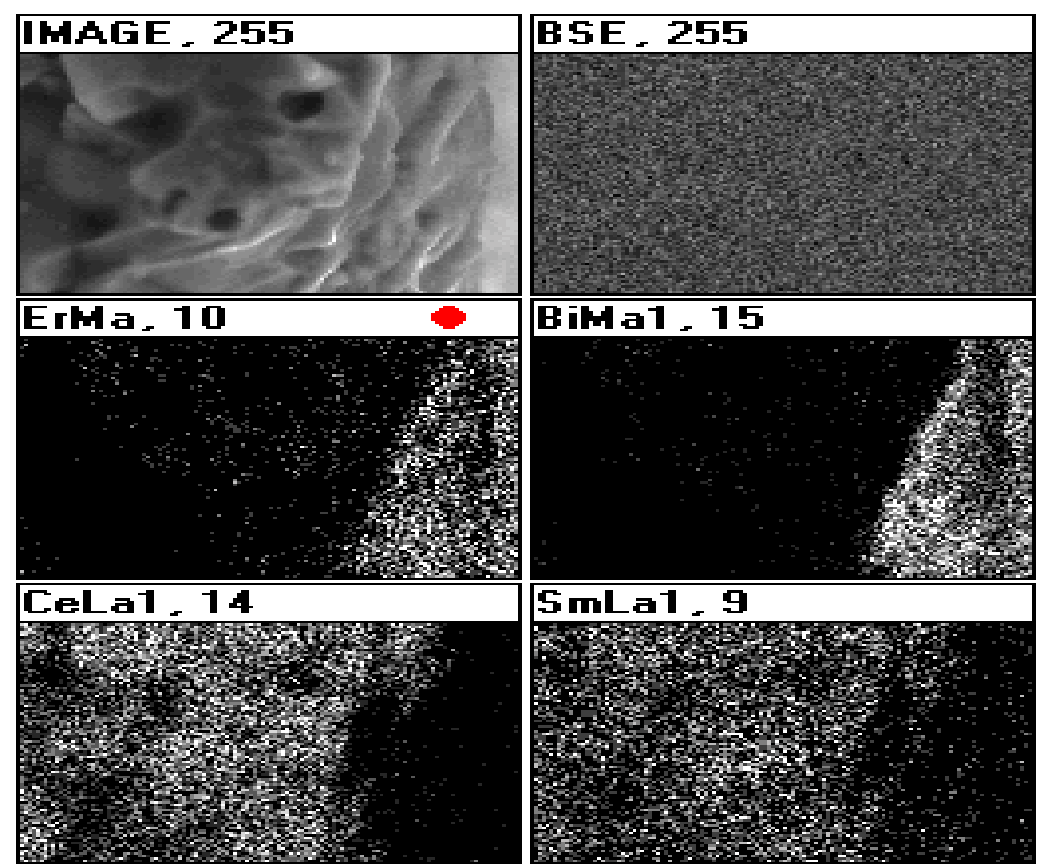

Bima1, 15

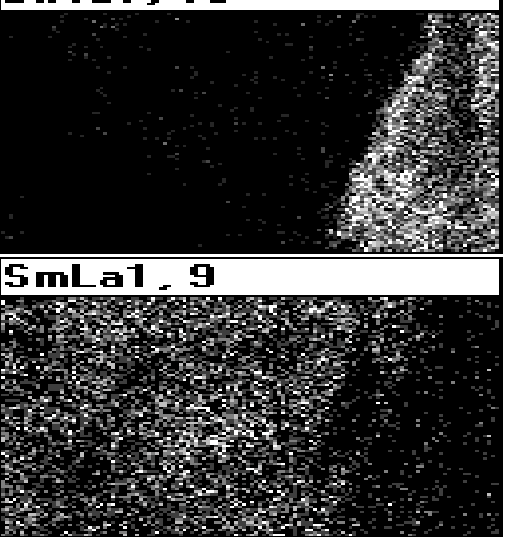

Figure 2-5(c). X-ray map of elements in an SDClESB sample (corresponding to Figure 2-5(b))

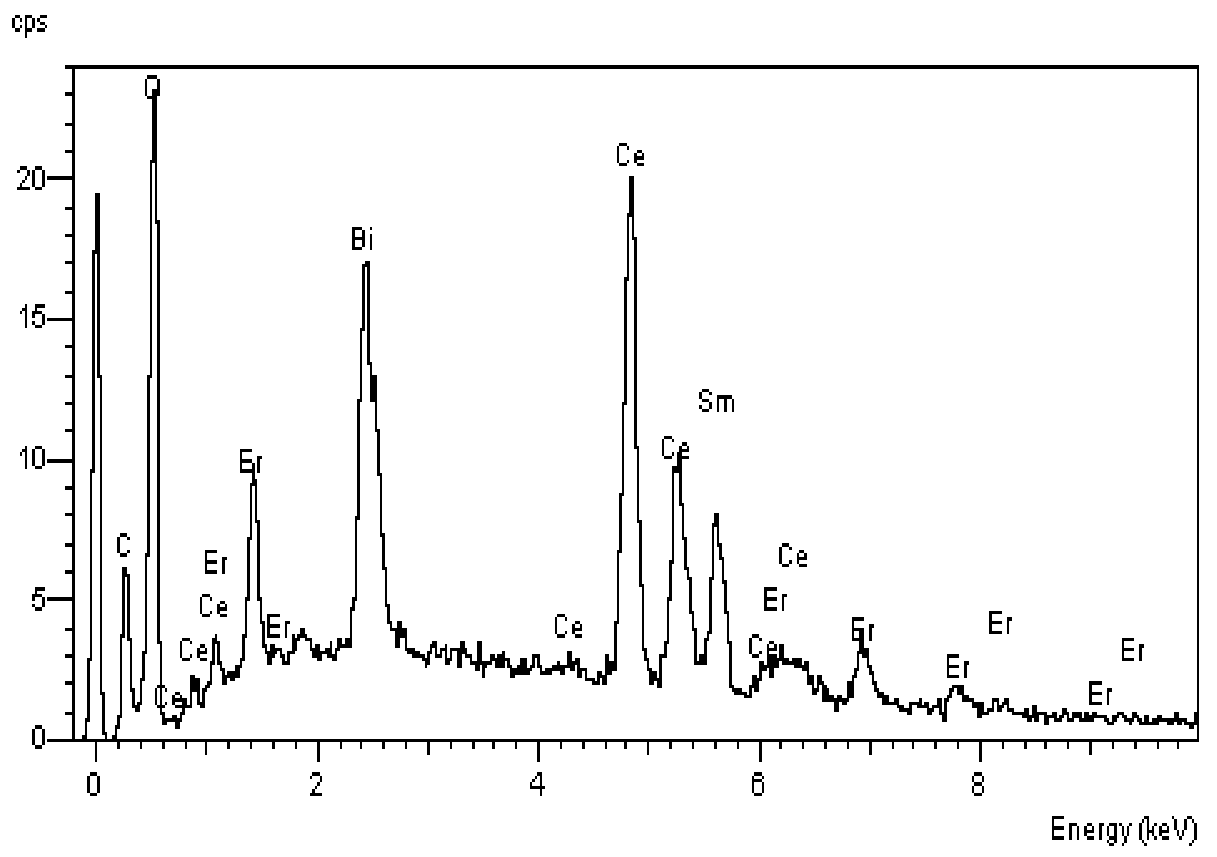

Figure 2-5(d). EDS spectra of the SDC\ESB sample cross-section.

Figure 2-5. The chemical composition of the bilayered SDCLESB electrolytes estimated from EDS by X-ray line-scan and mapping. 


\subsection{CONDUCTIVITY OF SDC}

\subsubsection{Analysis Methods}

Electrical impedance spectroscopy (EIS) can be used to measure the bulk, grainboundary, and electrode conduction processes by analysis of the consecutive semicircles in the complex plane. We used this technique to analyze the grain (or bulk) resistance, $R_{\mathrm{g}}$, and capacitance, $C_{\mathrm{g}}$, and the grain-boundary resistance, $R_{\mathrm{gb}}$, and capacitance, $C_{\mathrm{gb}}$, for various temperatures and microstructures. By combining the resistance and capacitance values with appropriate geometric information from the sample, the corresponding conductivities, $\sigma_{\mathrm{g}}$ and $\sigma_{\mathrm{gb}}$ were derived. This is neither a trivial nor an unambiguous operation, however, and there are currently two prevailing schools of thought that have produced two distinct methodologies namely: the geometric method and the capacitance method. Additional information may be obtained from recent reviews on grain-boundary conductivity and microstructure relations by Steele [19], Virkar et al. [20], Hong et al. [21], Tian and Chan [22], and Gerhardt and Nowick [23].

\subsubsection{Effect of Processing Parameters on SDC}

Sintering temperature and density. The ionic conductivity of SDC electrolytes sintered under various conditions was investigated and correlated with the resulting microstructure. Arrhenius plots of the ionic conductivities measured in air are shown in Figures 2-6. The slopes of the Arrhenius plots-obtained by plotting the following equation:

$$
\ln \sigma T=\ln \sigma^{\circ}-\frac{E_{A}}{k_{B}} \cdot \frac{1}{T}
$$

- give the activation energy, $E_{A}$, for transport of the oxygen ion. In Eq. (2.1), $\sigma^{\circ}$ is a preexponential factor, $k_{B}$ is Boltzmann's constant and $T$ is temperature

Figure 2-6 shows that variations in the sintering conditions and density of pellets can change their conductivity by as much as an order of magnitude. In general, the higher densities correspond to higher ionic conductivities. However, the ionic conductivity of $90 \%$ density SDC (sintered at $1550^{\circ} \mathrm{C}$ for $15 \mathrm{hrs}$ ) is higher than that of $98 \%$ density SDC (sintered at $1650^{\circ} \mathrm{C}$ for $15 \mathrm{hrs}$ ) in the temperature range $300-800^{\circ} \mathrm{C}$. That is, the conductivity increased with sintering temperature and time for the samples sintered below $\sim 1550^{\circ} \mathrm{C}$ and decreases at higher sintering temperatures. These phenomena are discussed in ensuing sections. 


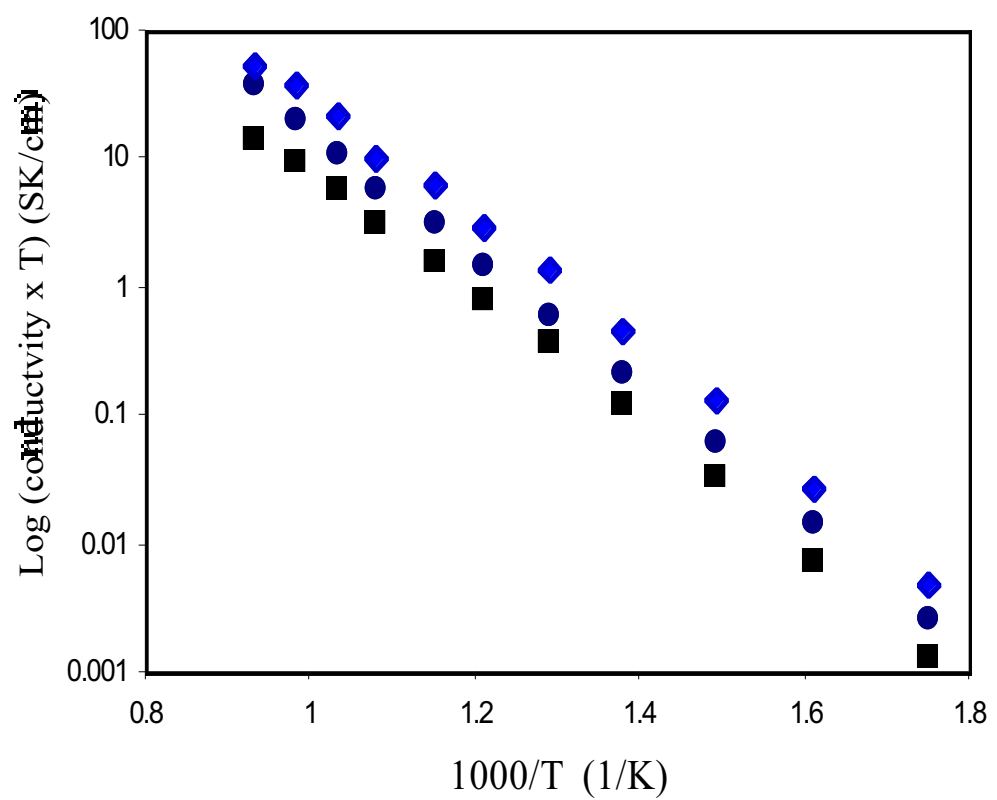

Figure 2-6. Total conductivities, in air, of SDC (uni-axial pressing) samples: $(\bullet)$ density, $97.5 \%$, sintering temperature, $1650^{\circ} \mathrm{C},(\diamond) 89.8 \%, 1550^{\circ} \mathrm{C}$, and $(\square) 70 \%, 1450^{\circ} \mathrm{C}$.

Cold-isostatic pressing. The effect of CIP on SDC samples can be observed by the change in $E_{A}$ at $650{ }^{\circ} \mathrm{C}$ in Figure 2-7. Figure 2-8 shows that this change was brought about by reduced grain-boundary resistance, for $T<650{ }^{\circ} \mathrm{C}$. Cold-isostatic pressing promotes grain growth, which in turn reduces the grain-boundary area (and therefore grain-boundary volume fraction, $\left.V_{g b}\right)$. Consequently, the contribution of the grain-boundary to the total conductivity, $\sigma_{t o t}$, decreases. The total conductivity ${ }^{2}$ is the sum of the grain (bulk) conductivity, $\sigma_{g}$, and grainboundary conductivity, $\sigma_{g b}$, as shown below:

$$
\sigma_{t o t}=\sigma_{g} V_{g}+\sigma_{g b} V_{g b}
$$

where $V_{g}$ is the grain (bulk) volume fraction. It is evident in Eq. (2.2) that if $\sigma_{g}>\sigma_{g b}$ (true for SDC) then $\sigma_{t o t}$ increases as $V_{g b}$ decreases. Conversely, above $650{ }^{\circ} \mathrm{C}$, Figure 2-7, the effect of a smaller $V_{g b}$ due to CIP is reduced significantly because differences between the values of $E_{A}$ and $\sigma^{0}$ for $\sigma_{g}\left(\right.$ lower $E_{A}$ ) and $\sigma_{g b}\left(\right.$ higher $E_{A}$ ) dominates at higher temperatures.

2 It is assumed that $\sigma_{g}>\sigma_{g b}$ and therefore parallel contributions to the total conductivity may be ignored. 


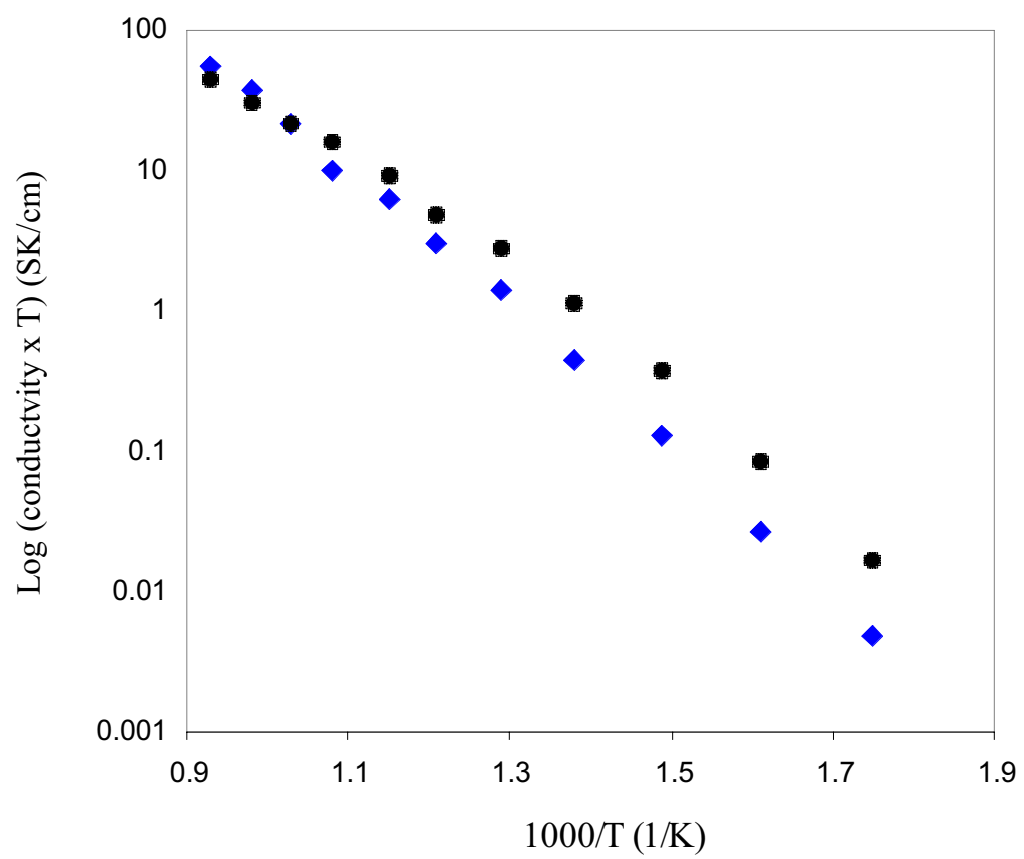

Figure 2-7. Effect of CIP on $\sigma_{\text {tot }}$ for SDC sintered at $1550{ }^{\circ} \mathrm{C}$ for $15 \mathrm{hrs}$ : $(\bullet) \mathrm{CIP}$ and $(\diamond)$ uniaxial pressing.

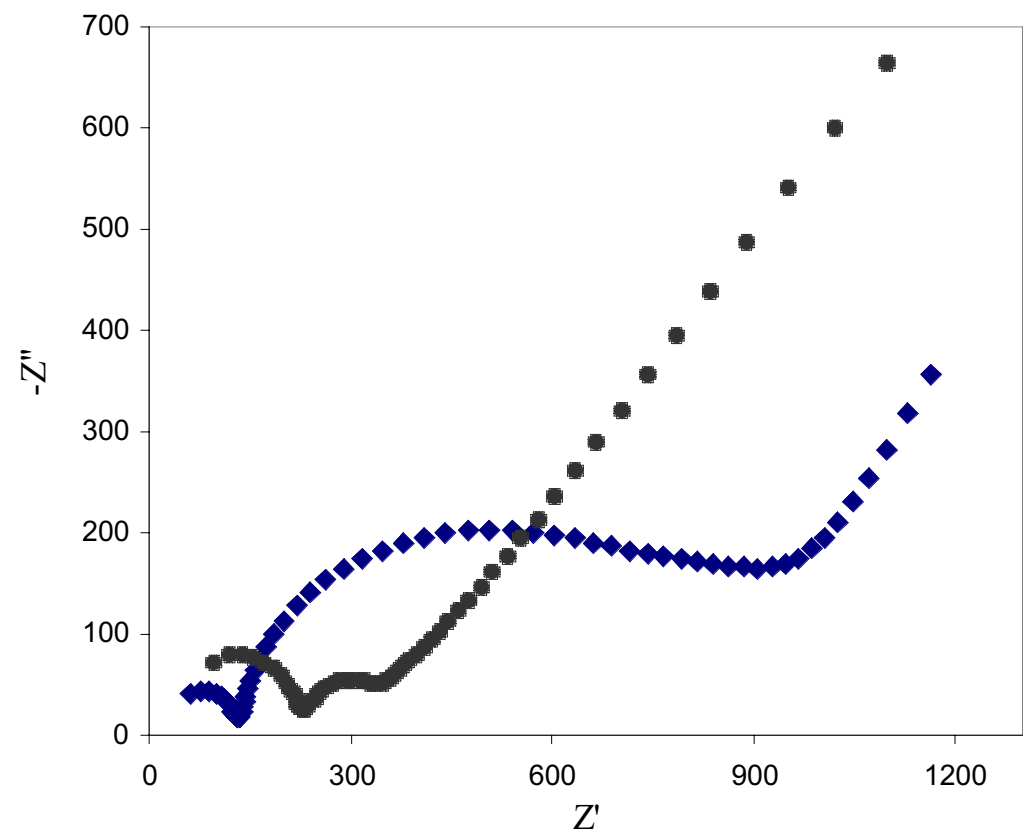

Figure 2-8. Impedance plots for SDC samples at $300{ }^{\circ} \mathrm{C} ;(\bullet) \mathrm{CIP}$ and $(\diamond)$ uni-axial pressing. 


\subsubsection{Effect of Grain-Boundary Impurities in SDC}

For ceramics, conductivity depends primarily on a variety of microstructural parameters, e.g. grain size and porosity. In particular, grain boundaries have an important effect on the overall conductivity of most ceramic materials and $\sigma_{\mathrm{gb}}$ is affected by factors such as grain size (discussed earlier) and impurity level, among others.

Impurities, including second phases, introduced by the starting materials or during the fabrication procedure have a deleterious effect on $\sigma_{\mathrm{gb}}$ of SDC [19] because they tend to accumulate at grain boundaries during sintering. Various studies $[19,24,25]$ have shown that continuous siliceous phases suppress grain growth resulting in small grain-sizes and larger grainboundary volume fraction (smaller grain volume fraction). Moreover, these impurities provide a blocking layer within the grain-boundary system that makes the grain-boundary region less conductive. Together these effects significantly reduce the ionic conductivity of ceria.

\subsubsection{Microstructure}

Grain-size distributions were estimated using a linear intercept method. They were found to vary with sintering temperature in the range $\sim 2$ to $\sim 15 \mu \mathrm{m}$ as shown in Table 2-1. Grainboundary thicknesses were procured from Christie and Berkel [26] whose data was obtained by high-resolution electron microscopy (HREM).

It is observed in Table 2-1 that as the sintering temperature increases so does the grainsize. Since the cations are not mobile until high temperature more than $1500{ }^{\circ} \mathrm{C}$, extensive grain growth occurs above $1500{ }^{\circ} \mathrm{C}$. In addition, high sintering temperatures are needed to ensure a homogeneous distribution of dopants in the sample. This concurs with the notion that cation transport is the limiting step for grain growth [27]. However, low cation mobility in the fluorite lattice means that cation segregation-formed by temperature gradients-may arise at elevated temperatures. Accordingly, it was found that changes in the sintering temperature and time can produce variations in both and bulk and grain-boundary conductivity by more than an order of magnitude. That is, sintering conditions have a significant effect on SDC conductivity.

Table 2-1 Mean Grain Size by Different Sintering Temperatures.

\begin{tabular}{ccc}
\hline Sintering temperature $\left({ }^{\circ} \mathrm{C}\right)$ & Sintering time $($ hrs. $)$ & Mean grain size $(\mu \mathrm{m})$ \\
\hline 1465 & 15 & 1.84 \\
1550 & 15 & 4.21 \\
1650 & 15 & 15.3 \\
\hline
\end{tabular}




\subsection{CONDUCTIVITY OF ESB AND DWSB}

Impedance using the two probe method was measured with a Solartron 1260 over the frequency range of $0.1 \mathrm{~Hz}$ to $1 \mathrm{MHz}$. The frequency response analyzer was interfaced to a computer, and Zplot software and a nulling technique were employed to remove any artifacts caused by inductive responses of the test leads and the equipment. The measurements were taken between $200^{\circ} \mathrm{C}$ and $800^{\circ} \mathrm{C}$ in air. The flow rate of air was fixed at $30 \mathrm{sccm}$. Activation energies were calculated from plots of $\ln (\sigma \mathrm{T})$ vs. $1 / \mathrm{T}$.

Figure 2-9 shows the impedance spectra for 8 Dy4WSB at 200 (a), 300 (b) and $500{ }^{\circ} \mathrm{C}$ (c). The conductivities were calculated from the values of the bulk resistance measured at the frequency corresponding to the appropriate minima in the complex plots. Both Figure 2-9 (a) and Figure 2-9 (b) show the typical bulk impedance consisting of a single semicircle, and a Warberg-type impedance indicating gas diffusion at the electrode. As the temperature increased (Figure 2-9 (c)), the high frequency semicircle attributable to the bulk resistance could no longer be resolved. The bulk resistance values were then calculated from the high frequency intercept.

The bulk conductivity results are plotted as $\log \sigma$ vs. 1000/T (Figure 2-10). The compositions having 11-13\% dopant (10Dy3WSB, 8Dy4WSB and 6Dy5WSB) showed higher conductivity than 17\% dopant level(12Dy5WSB, 9Dy8WSB and 5Dy12WSB). A comparison of the conductivity of DyWSB and 20ESB is also shown in Figure 2-10. 20ESB is considered in the literature to have the highest conductivity among the stabilized $\delta-\mathrm{Bi}_{2} \mathrm{O}_{3}$ 's, with a conductivity at $800{ }^{\circ} \mathrm{C}, 500{ }^{\circ} \mathrm{C}$ and $200{ }^{\circ} \mathrm{C}$ of $0.41,0.021$ and $2.8 \times 10^{-5} \mathrm{~S} / \mathrm{cm}$, respectively. The conductivities of $8 \mathrm{Dy} 4 \mathrm{WSB}$, the highest conductivity composition, at $800{ }^{\circ} \mathrm{C}, 500{ }^{\circ} \mathrm{C}$ and 200 ${ }^{\circ} \mathrm{C}$ are $0.56,0.043$ and $1.4 \times 10^{-4} \mathrm{~S} / \mathrm{cm}$, respectively, significantly higher than 20ESB, especially in the lower temperature region.

A change in activation energy for some of the compositions was observed, with a lower activation energy above $\sim 600^{\circ} \mathrm{C}$ and a higher activation energy below $\sim 600^{\circ} \mathrm{C}$. As the dopant concentration increases, the disparity between these two activation energies decreases. The observed disparity at $\sim 600^{\circ} \mathrm{C}$ as a function of dopant concentration is a general trend in stabilized bismuth oxide systems [9]. The activation energy values measured for low and high temperatures with different compositions are shown in Table 1 and 2. From Table 1 and Figure 2-10, it is clear that the lower dopant content compounds (11-13\% dopant) showed two different conductivity activation energies. On the other hand, the conductivity of $17 \%$ dopant level compositions (Table 2 and Figure 2-10) showed single activation energies, except for 12Dy5WSB that exhibited a slight change in activation energy. This change in activation energy is attributed to an order-disorder transition of the oxygen sublattice [11, 16]. Those compositions 
with the greatest disparity have the greatest tendency to order at low temperature [9]. This tendency and the resulting conductivity decay are described in another publication [28].

In Figure 2-11, the conductivity in the $\mathrm{WO}_{3}-\mathrm{Dy}_{2} \mathrm{O}_{3}-\mathrm{Bi}_{2} \mathrm{O}_{3}$ system is plotted against the dopant composition ratio $\left(\mathrm{X}_{\mathrm{Dy}} /\left(\mathrm{X}_{\mathrm{Dy}}+\mathrm{X}_{\mathrm{W}}\right)\right)$ content at 500 and $700^{\circ} \mathrm{C}$, respectively. Values from the literature were used for comparisons at either end of the dopant composition ratio range $\left(\mathrm{WO}_{3}\right.$ stabilized $\mathrm{Bi}_{2} \mathrm{O}_{3}$ and $\mathrm{Dy}_{2} \mathrm{O}_{3}$ stabilized $\mathrm{Bi}_{2} \mathrm{O}_{3}$ ). $12 \mathrm{WSB}$ exhibits the highest conductivity among the phase stable $\mathrm{WO}_{3}$ compositions [13]. A thermal hysteresis in conductivity was observed at high temperature $\left(650\right.$ to $\left.750^{\circ} \mathrm{C}\right)$ for samples containing less than $20 \mathrm{~mol}_{\%} \mathrm{WO}_{3}$ $\left(\left(\mathrm{BiO}_{1.5}\right)_{0.89}\left(\mathrm{WO}_{3}\right)_{0.11}\right)$ due to the transformation from the monoclinic to the cubic structure. Therefore, the conductivity of 12WSB from Takahashi et al [15] is plotted (closed square) in Figure 2-11.

Verkerk and Burggraaf [16] investigated DySB, in the composition range 5 to $60 \mathrm{~mol} \%$ $\mathrm{Dy}_{2} \mathrm{O}_{3}$. The cubic phase for DySB containing 28.5 to $50 \mathrm{~mol} \% \mathrm{Dy}_{2} \mathrm{O}_{3}$ was found to be stable over the temperature of measurement $\left(300\right.$ to $\left.800^{\circ} \mathrm{C}\right)$. The samples with lower $\mathrm{Dy}_{2} \mathrm{O}_{3}$ content showed a phase transition from cubic to rhombohedral below $800^{\circ} \mathrm{C}$ and no conductivity data was available. Therefore, values of $28.5 \mathrm{DySB}$ are plotted (closed triangle) on the right axis in Figure 2-11 to compare with the doubly doped system, rather than comparing with the concentration range where polyphasic regions appear.

One of the effects of double doping $\mathrm{W}$ and Dy is that the cubic structure is stabilized at dopant concentrations less than that necessary for Dy alone. Thus, the cubic structure was stabilized at as little as a $11-13 \%$ dopant concentration.

At the higher total dopant concentration (17\%), intermediate between the dopant concentration of end compositions from the literature, there appears to be a minimum in conductivity at $\mathrm{X}_{\mathrm{Dy}} /\left(\mathrm{X}_{\mathrm{Dy}}+\mathrm{X}_{\mathrm{W}}\right)=0.4-0.5$ depending on temperature. In contrast, at the lower total dopant concentration (11-13\%), more closely matching the dopant concentration of the $\mathrm{W}$ end composition, there appears to be a maximum in conductivity at $\mathrm{X}_{\mathrm{Dy}} /\left(\mathrm{X}_{\mathrm{Dy}}+\mathrm{X}_{\mathrm{W}}\right)=0.5-0.6$, depending on temperature. These results indicate the nature of the dopant (W vs. Dy) is as important as the total dopant concentration and that the effect of double doping on conductivity is "non ideal." The observed differences in conductivity dependence on dopant ratio warrants further investigation.

In summary, the conductivity of $\mathrm{Dy}_{2} \mathrm{O}_{3}$ and $\mathrm{WO}_{3}$ stabilized $\mathrm{Bi}_{2} \mathrm{O}_{3}$ was studied. These cations were selected for their high polarizability in order to achieve a stable disordered anion lattice and a high mobility. By double doping we were able to obtain compositions (11-17\% dopant) that were cubic, when single dopants would not stabilize the cubic structure at this low 


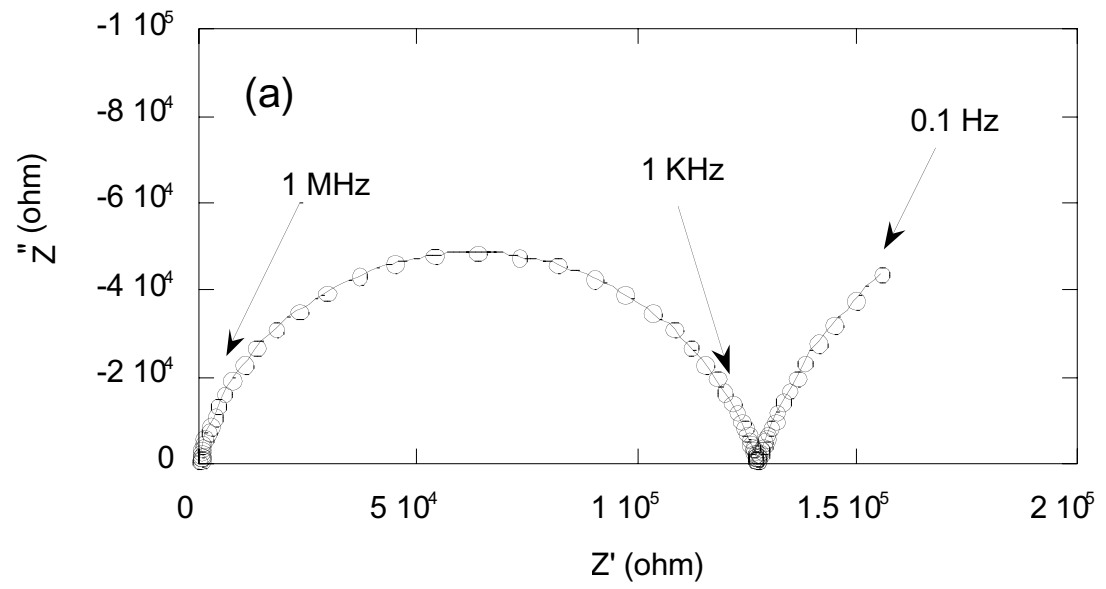

(a)

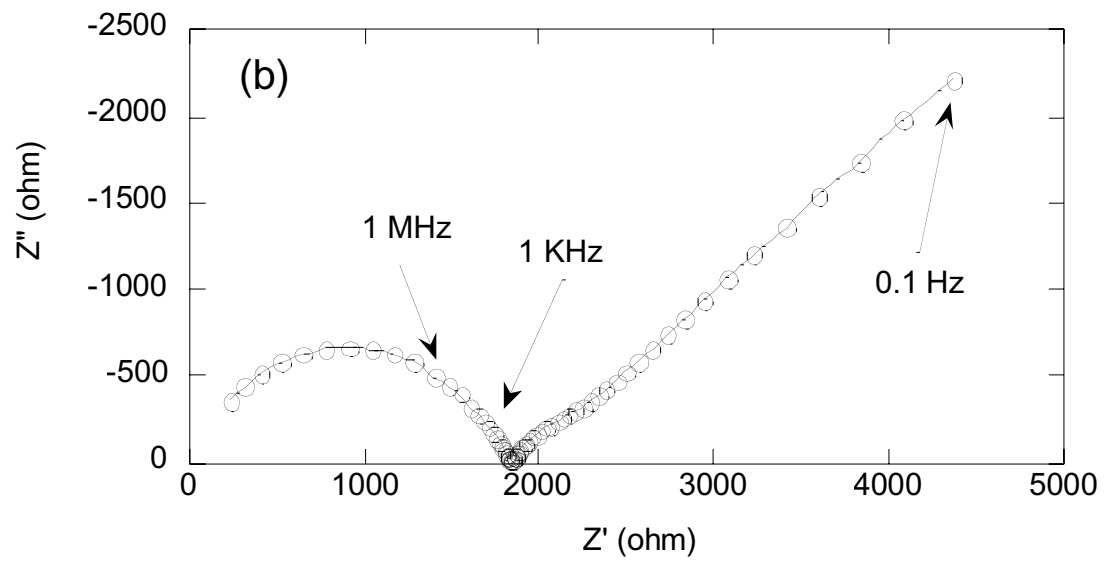

(b)

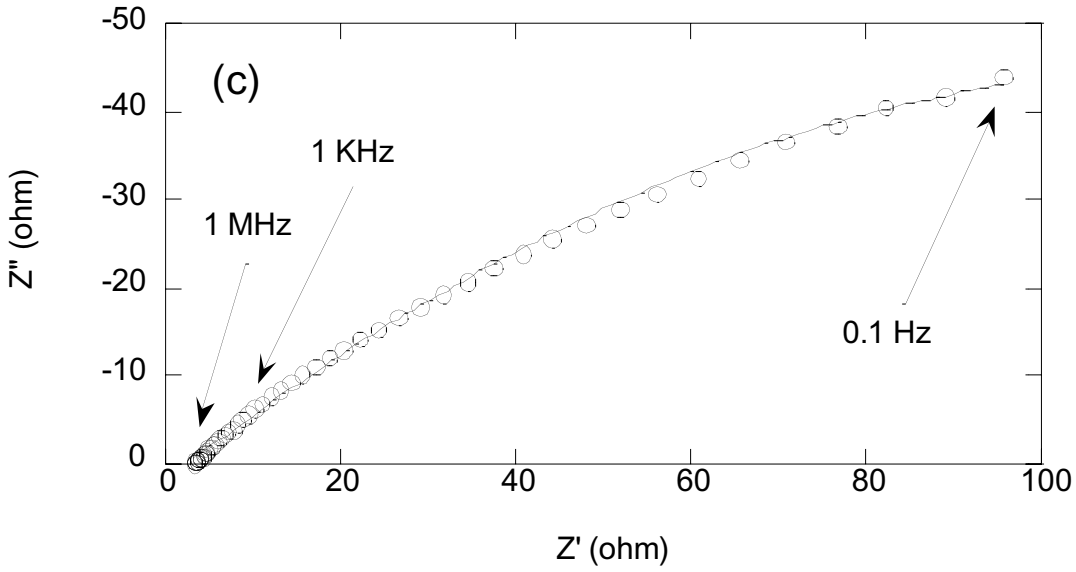

(c)

Figure 2-9. Impedance spectra for 8 Dy4WSB with Au electrodes in air at (a) $200^{\circ} \mathrm{C}$ (b) $300^{\circ} \mathrm{C}$ and (c) $500^{\circ} \mathrm{C}$. 


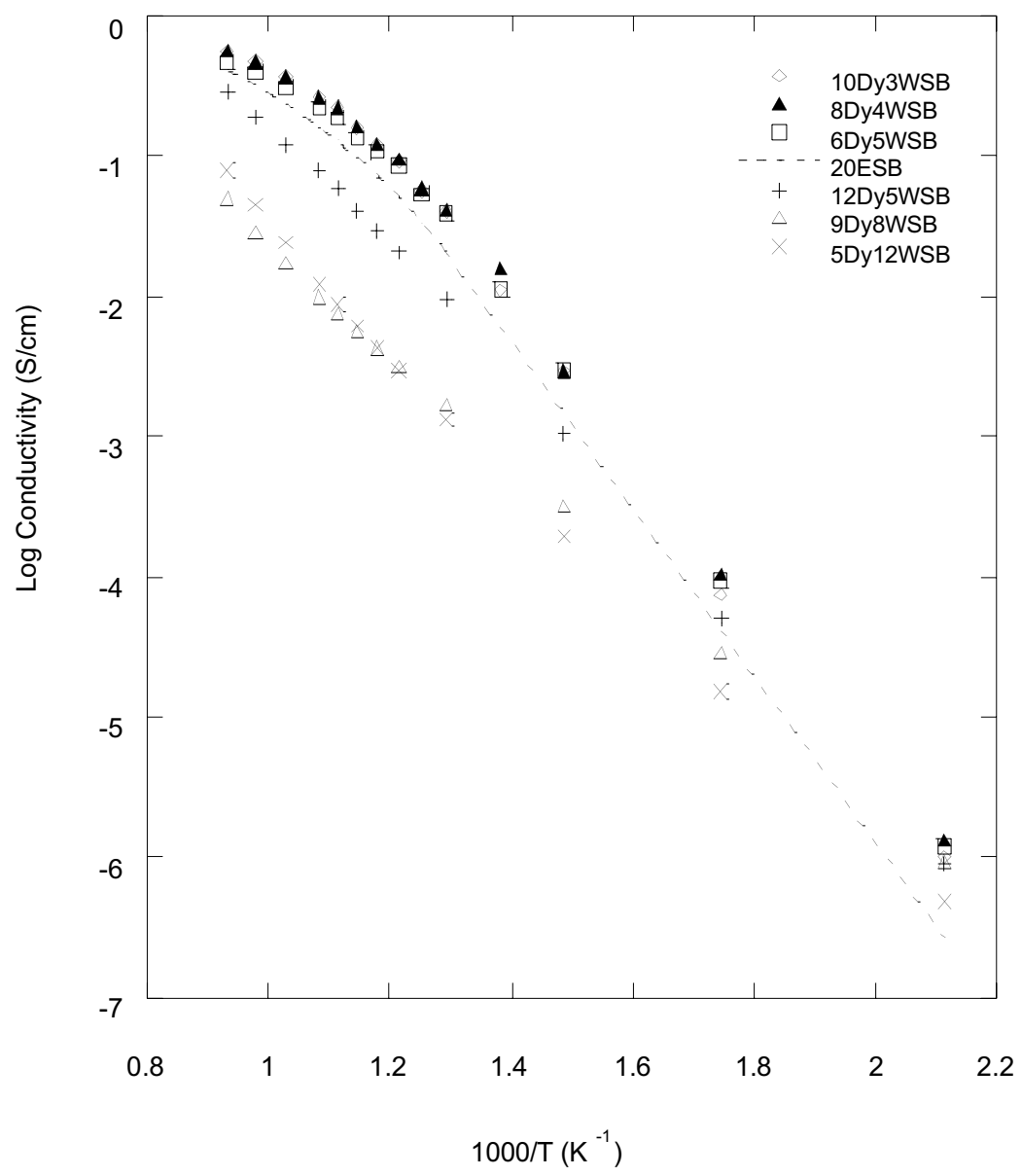

Figure 2-10. Arrhenius plot of conductivities for 10Dy3WSB, 8Dy4WSB, 6Dy5WSB, 12Dy5WSB, 9Dy8WSB, 5Dy12WSB and 20ESB

Table 1. Conductivity activation energies of $11-13 \%$ total dopant compositions

\begin{tabular}{ccc} 
Composition & Low Temperature $\left(<600{ }^{\circ} \mathrm{C}\right) \mathrm{E}_{\mathrm{A}}(\mathrm{eV})$ & High Temperature $\left(>600{ }^{\circ} \mathrm{C}\right) \mathrm{E}_{\mathrm{A}}(\mathrm{eV})$ \\
\hline 6Dy5WSB & 0.47 & 0.23 \\
8Dy4WSB & 0.47 & 0.29 \\
10Dy3WSB & 0.48 & 0.19 \\
\hline
\end{tabular}

Table 2. Conductivity activation energies of $17 \%$ total dopant compositions

\begin{tabular}{ccc}
\hline Composition & Low Temperature $\left(<600{ }^{\circ} \mathrm{C}\right) \mathrm{E}_{\mathrm{A}}(\mathrm{eV})$ & High Temperature $\left(>600{ }^{\circ} \mathrm{C}\right) \mathrm{E}_{\mathrm{A}}(\mathrm{eV})$ \\
\hline 5Dy12WSB & 0.39 & 0.39 \\
9Dy8WSB & 0.34 & 0.34 \\
12Dy5WSB & 0.42 & 0.34 \\
\hline
\end{tabular}



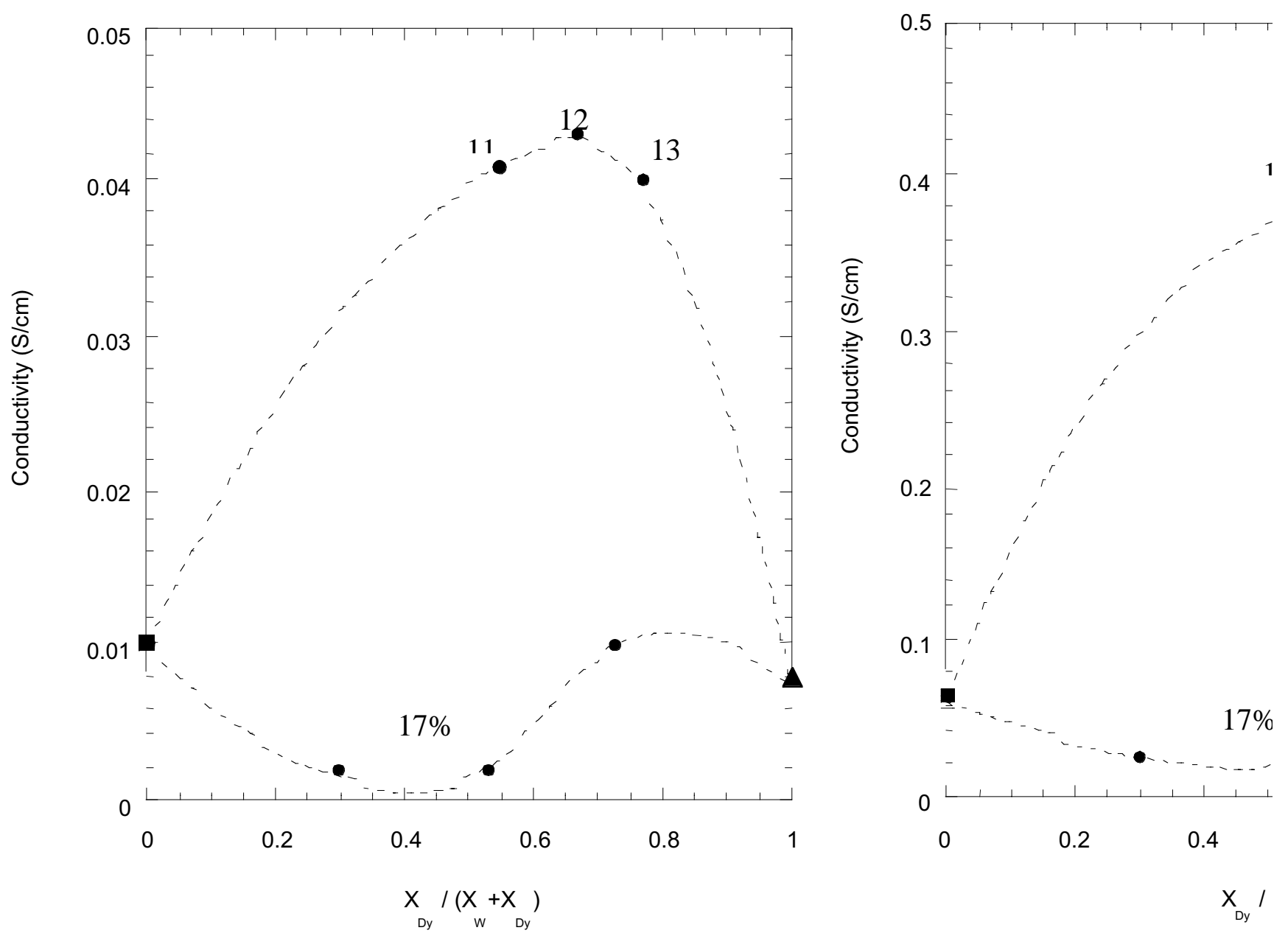

(a)

Figure 2-11. Conductivity as a function of dopant composition ratio $\left(\mathrm{X}_{\mathrm{Dy}} /\left(\mathrm{X}_{\mathrm{Dy}}+\mathrm{X}_{\mathrm{W}}\right)\right)$ and total dopant concentration at $(\mathbf{a}) 500^{\circ} \mathrm{C}$ and $(\mathbf{b}) 700^{\circ} \mathrm{C}$. The conductivity of $12 \mathrm{WSB}$ (Takahashi et al [15], closed square) and 28.5DySB (Verkerk et al [16], closed triangle) is given as a reference at either end of the range. 
of a dopant level. The resultant cubic DyWSB's had high conductivity. In particular, the composition 8Dy4WSB had a conductivity significantly greater than 20ESB, rating it the most conductive oxide electrolyte currently known.

\subsection{CONDUCTIVITY OF SDC\ESB BILAYERS}

The effective ${ }^{3}$ conductivities of SDClESB sample (ESB film thickness: $\sim 0.2 \mu \mathrm{m}$ ) were measured in air by EIS. Figure 2-12 shows that the effective conductivity of the SDCLESB bilayer was higher than that of single layer SDC (fabricated by CIP or uniaxial pressing) for 600 ${ }^{\circ} \mathrm{C}<\mathrm{T}<800{ }^{\circ} \mathrm{C}$ despite the relative thinness of the ESB layer-i.e., $\sigma_{\mathrm{SDClESB}}>\sigma_{\mathrm{SDC}(\mathrm{CIP})}>$ $\sigma_{\mathrm{SDC}(\text { uniaxial) }}$. This result also suggests that a low conductivity (relative to SDC and ESB) phase is not formed at the SDC-ESB interface. Nevertheless, because of the thinness of the ESB layer, the increase in the effective conductivity of the bilayer over the SDC single layer is somewhat surprising and may allude to the formation of a higher conductivity ESB phase in the grainboundary area of SDC. This is speculative, however, and requires further research for confirmation.

The change in $E_{A}$ for the conductivity of the SDC \ESB sample, seen at $600^{\circ} \mathrm{C}$ in Figure 212 , may be attributed to the order-disorder transition in the oxygen sublattice of $\mathrm{ESB}$ at $600^{\circ} \mathrm{C}$ $[29,30]$. We have studied this and the time dependent ageing phenomenon in phase-stabilized bismuth oxides extensively $[29,30]$.

Activation energies and pre-exponential factors for SDC and SDC listed in Table 2-2 and they are in good agreement with Tian and Chan [22], and Wang et al.'s results [31]. An inspection of Table 2-2 shows that activation energies and pre-exponential factors obtained by the capacitance method [19 - 23] were larger than those obtained by the geometric method [19 - 23].

A similar difference between the two methods was observed by Tian and Chan [22]. The difference between the values of the activation energy and pre-exponential terms, obtained from the capacitance and geometric methods, most likely arises from the inaccuracy in the assumed values of grain size and grain-boundary thickness used in the geometric method [19 - 23].

3 Conductivity is an intrinsic material property, independent of sample geometry. Hence a bilayer does not have a true conductivity. The term effective conductivity is used here to describe a hypothetical material with a conductivity that is equivalent to the bilayer. 
Table 2-2. Activation energies and pre-exponential factors of various SDC and SDCIESB samples.

\begin{tabular}{|c|c|c|c|c|c|c|}
\hline Sample thickness; $(\mathrm{cm})$ & $\begin{array}{c}\text { Relative } \\
\text { Density } \\
(\%) \\
\end{array}$ & CIP & $\begin{array}{l}\mathrm{T}_{\text {sinter }} \\
\left({ }^{\circ} \mathrm{C}\right)\end{array}$ & $\begin{array}{c}\text { Conductivity } \\
\text { Type }\end{array}$ & $\begin{array}{c}\text { Activation } \\
\text { energy } \\
(\mathrm{eV})\end{array}$ & $\begin{array}{c}\text { Pre- } \\
\text { exponential } \\
(\mathrm{SK} / \mathrm{cm})\end{array}$ \\
\hline \multirow[t]{4}{*}{ SDC (0.172) } & 97.5 & No & 1650 & Total & $0.99(3)$ & $2.0 \times 10^{5}$ \\
\hline & & & & Bulk & $0.85(1)$ & $2.0 \times 10^{6}$ \\
\hline & & & & Grain $(G)^{1}$ & $1.0(11)$ & $2.5 \times 10^{4}$ \\
\hline & & & & $\operatorname{Grain}(\mathrm{C})^{2}$ & $1.7(01)$ & $4.0 \times 10^{8}$ \\
\hline \multirow[t]{4}{*}{$\operatorname{SDC}(0.183)$} & 89.5 & No & 1550 & Total & $0.97(5)$ & $3.0 \times 10^{6}$ \\
\hline & & & & Bulk & $0.86(9)$ & $3.0 \times 10^{6}$ \\
\hline & & & & Grain (G) & $1.0(54)$ & $1.8 \times 10^{4}$ \\
\hline & & & & Grain $(\mathrm{C})$ & $1.8(65)$ & $1.0 \times 10^{11}$ \\
\hline \multirow[t]{4}{*}{$\operatorname{SDC}(0.145)$} & 70.0 & No & 1465 & Total & $0.97(7)$ & $6.8 \times 10^{5}$ \\
\hline & & & & Bulk & $0.75(8)$ & $1.3 \times 10^{5}$ \\
\hline & & & & Grain $(\mathrm{G})$ & $1.0(57)$ & $7.7 \times 10^{4}$ \\
\hline & & & & Grain $(\mathrm{C})$ & $1.8(65)$ & $1.0 \times 10^{11}$ \\
\hline \multirow[t]{5}{*}{$\operatorname{SDC}(0.171)$} & 90.4 & Yes & 1550 & Total $^{3}$ & $0.97(0)$ & $6.0 \times 10^{6}$ \\
\hline & & & & Total $^{4}$ & $0.66(5)$ & $2.0 \times 10^{7}$ \\
\hline & & & & Bulk & $0.77(7)$ & $3.8 \times 10^{5}$ \\
\hline & & & & Grain $(\mathrm{G})$ & $0.98(4)$ & $2.5 \times 10^{4}$ \\
\hline & & & & Grain (C) & $1.7(53)$ & $1.0 \times 10^{11}$ \\
\hline \multirow[t]{5}{*}{$\operatorname{SDC\backslash ESB}\left(0.17 \backslash 2 \times 10^{-5}\right)$} & 90.4 & Yes & 1550 & Total $^{3}$ & $1.0(39)$ & $2.0 \times 10^{7}$ \\
\hline & & & & Total $^{4}$ & $0.66(5)$ & $6.0 \times 10^{4}$ \\
\hline & & & & Bulk & $0.91(8)$ & $5.0 \times 10^{6}$ \\
\hline & & & & Grain $(\mathrm{G})$ & $0.91(3)$ & $8.0 \times 10^{3}$ \\
\hline & & & & Grain (C) & $1.8(65)$ & $1.0 \times 10^{11}$ \\
\hline
\end{tabular}

1. Grain $(\mathrm{G})$ : grain-boundary conductivity obtained by using geometrical method.

2. Grain (C): grain-boundary conductivity obtained by using grain and grain-boundary capacitance

3. Total: total conductivity in temperature range between at $300{ }^{\circ} \mathrm{C}$ and $500{ }^{\circ} \mathrm{C}$.

4. Total: total conductivity in temperature range between at $500^{\circ} \mathrm{C}$ and $800^{\circ} \mathrm{C}$. 


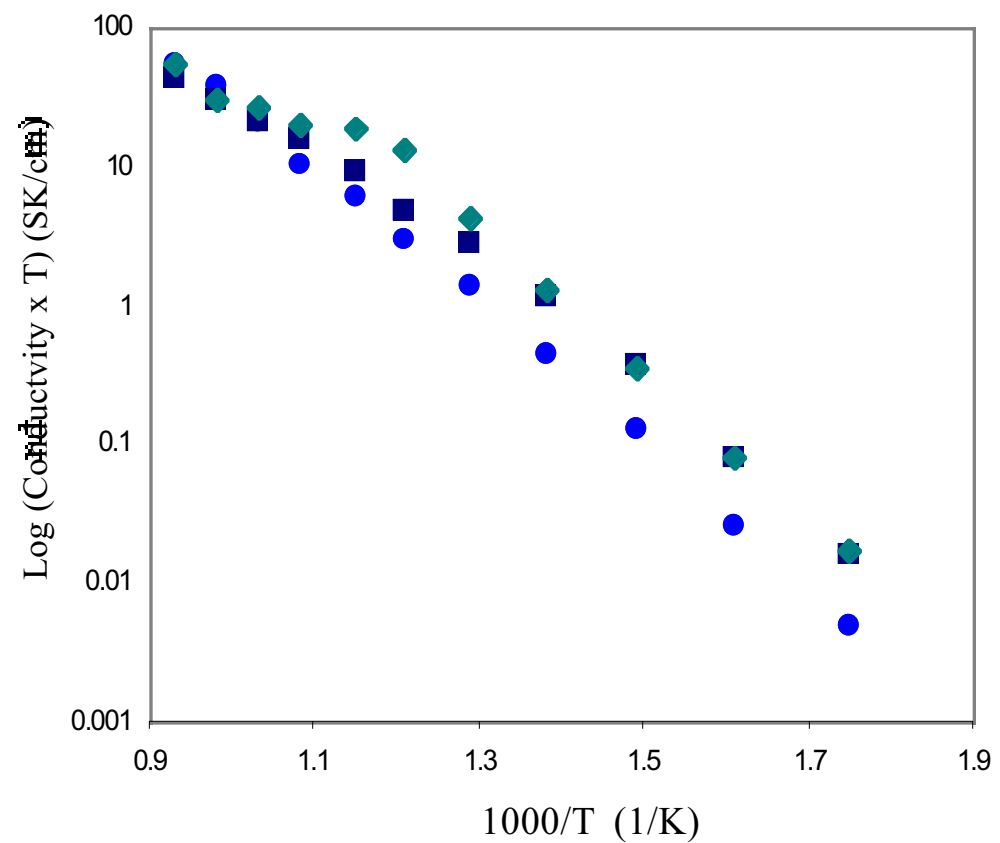

Figure 2-12. Total conductivity, in air, of $(\bullet)$ SDC (density: $90 \%$, uni-axial pressing, sintered at $\left.1550{ }^{\circ} \mathrm{C}\right),(\square) \mathrm{SDC}\left(90 \%, \mathrm{CIP}, 1550{ }^{\circ} \mathrm{C}\right)$ and $(\diamond) \operatorname{SDClESB}(90 \%, \mathrm{CIP})$.

\subsection{FABRICATION OF ANODE-SUPPORTED CERIA ELECTROLYTES BY COLLOIDAL DEPOSITION}

Investigation into the effect of relative thickness of ceria-bismuth oxide bilayers can be facilitated by electrode-supported electrolytes, as fabrication of the bilayers requires some ingenuity due to the large difference in the sintering temperatures of bismuth oxide and ceria. As a first step, the fabrication and performance of Ni-gadolinium doped ceria (GDC) anode supported GDC thick film electrolyte, using a colloidal deposition method is being reported here. Future work involves depositing doped bismuth oxide layer on the GDC coated anode substrates, to form anode supported bilayer electrolytes and electrochemical characterizations on them. An anode supported electrolyte cell was preferred over a cathode supported electrolyte cell as the anode polarization is, in general, smaller than the cathode polarization. GDC as the ceramic component in the anode will lead to an enhanced performance, as under the reducing conditions at the anode GDC is a mixed ionic electronic conductor and the reaction zone will not be restricted to the interface of the anode and the electrolyte.

Microstructure plays an important role in the performance of the electrodes, as shown in a recent work by Ohara et al [32]. They studied the performance of Ni-Samaria doped Ceria 
cermet anodes as a function of $\mathrm{Ni}$ content and found that at $800^{\circ} \mathrm{C}$, the lowest anodic polarization $(\sim 30 \mathrm{mV})$ was achieved for a cermet with a Ni content of around $50 \mathrm{vol} \%$ at a current density of $300 \mathrm{~mA} / \mathrm{cm}^{2}$. Thus, in this work a NiO-GDC anode was fabricated with Ni content of 50vol\%. Reduction of $\mathrm{NiO}$ to $\mathrm{Ni}$ was accomplished in situ in the cell, which would generate $~ 26 \%$ porosity in the structure.

Several techniques have been developed for depositing thick films on substrates from vapor deposition techniques (CVD/EVD) and pulse laser deposition (PLD) to chemical routes (sol-gel) among others [33]. However, colloidal deposition has definite advantages over these techniques in its simplicity, cost-effectiveness, upscability and flexibility (thickness ranging from 10-100 $\mu \mathrm{m}$ can be deposited). Also, exceptional performance has been reported for SOFCs fabricated from colloidal deposition technique by de Souza et al. using a $10 \mu \mathrm{m}$ thick YSZ electrolyte [34].

\subsubsection{Experimental}

$11 \mathrm{~mol} \%$ GDC powder (GDC $\mathrm{d}_{50}=0.64 \mathrm{um}$ ) from Rhodia and NiO powder from AlfaAesar were mixed and ball milled for 24 hours with Zirconia ball media in Ethanol. The mixed $\mathrm{NiO}-\mathrm{GDC}$ powder was pressed uniaxially at pressures of $39.3 \mathrm{MPa}$ to form discs of 1.25 inches diameter. The green bodies were then pre-sintered at temperatures between $800^{\circ} \mathrm{C}$ to $1100^{\circ} \mathrm{C}$, to coarsen the microstructure and to provide strength for further processing steps.

For the colloidal suspensions, 10g of GDC powder was mixed in 100ml of Iso-propyl alcohol (IPA). Poly-vinyl Butyral (PVB) in ethanol was added to the solution as binder, equal to $5 \mathrm{wt} \%$ of the oxide powder. The suspension was sonicated in an ultrasonic bath to disperse the GDC powder, with intermittent stirring. The GDC electrolyte layer was deposited on the presintered NiO-GDC discs by dip-coating and finally sintered at $1600^{\circ} \mathrm{C}$ and $1650^{\circ} \mathrm{C}$. Multiple coating was done to get thicker films.

The pre-sintering and final sintering temperatures were varied to find the optimum combination at which the densification rate of the GDC film matches with the shrinkage rate of the NiO-GDC anode substrate. This helps in keeping the film under compression rather than in tension and hence, avoids the cracking of the film due to stress built up by the 2-D confinement during sintering.

The diameters of the anode discs were measured after each processing step to calculate the shrinkage profile. The density of the discs was measured using the Archimedes's principle. The coated substrate was characterized by XRD (APD-4720) and SEM (JEOL-6400) techniques. 
The percentage porosity of the GDC films was measured by stereological counting methods using point probes.

\subsubsection{Results and Discussion}

The representative microstructures of the ceria film with the anode substrates pre-sintered at $800 \mathrm{C}, 850^{\circ} \mathrm{C}, 900^{\circ} \mathrm{C}, 1000^{\circ} \mathrm{C}, 1100^{\circ} \mathrm{C}$ for 4 hours, and after coating finally sintered at $1600^{\circ} \mathrm{C}$ for 6 hours and $1650^{\circ} \mathrm{C}$ for 10 hours are show in Figure 2-13(a)-(e) and Figure 2-14(a)-(e), respectively. Figure 2-15 shows the shrinkage profile of the NiO-GDC substrate during sintering. The density of the anode substrate was calculated to be $6.72 \mathrm{gm} / \mathrm{cc}$ ( $98 \%$ of the theoretical density) after sintering at $1600^{\circ} \mathrm{C}$. Figure $2-16$ shows the percentage porosity of the GDC film under different sintering profiles. The volume fraction of the porosity in the microstructure is calculated by taking the average fraction of points on a grid, lying on the pores. Figure 2-17 shows the cross-sectional SEM of the film pre-sintered at $900^{\circ} \mathrm{C}$ for 4 hours and finally sintered at $1600^{\circ} \mathrm{C}$ for 6 hours. The thickness of the film was about $10 \mu \mathrm{m}$ after 4 coats.

For applications in SOFCs, the electrolyte needs to be highly dense to act as a barrier for the fuel gases and air. As can be seen from Figure 2-16, varying the pre-sintering temperatures lead to minima in porosity at $850^{\circ} \mathrm{C}$ for both the final sintering temperatures of $1600^{\circ} \mathrm{C}$ and $1650^{\circ} \mathrm{C}$, with values of $1.38 \%$ and $1.48 \%$ respectively. Therefore, after pre-sintering at $850^{\circ} \mathrm{C}$, the shrinkage rate of the anode matches the densification rate of the film. However, as can be seen in the Figure $2-14$, sintering at $1650^{\circ} \mathrm{C}$ leads to coarsening of the microstructure. Both the grain and pore size increase and become almost the double the size to that sintered at $1600^{\circ} \mathrm{C}$. Bigger pore size in the electrolyte microstructure has larger chances of failure in partitioning the fuel and air atmospheres in comparison. Also, thermodynamic studies on film stability have shown that, the film thickness should be larger than the grain size of the film [35]. Otherwise, the film would lower its energy by breaking into isolated islands and exposing the substrate. Hence, it was decided to work with films pre-sintered at $850^{\circ} \mathrm{C}$ and finally sintered at $1600^{\circ} \mathrm{C}$ for OCP and I-V measurements. Figure 2-17 shows the cross-sectional SEM of the film pre-sintered at $850^{\circ} \mathrm{C}$ for 4 hours and finally sintered at $1600^{\circ} \mathrm{C}$ for 6 hours, after being tested in a fuel cell for over 200 hours showing the reduced porous anode and the dense Ceria layer. 


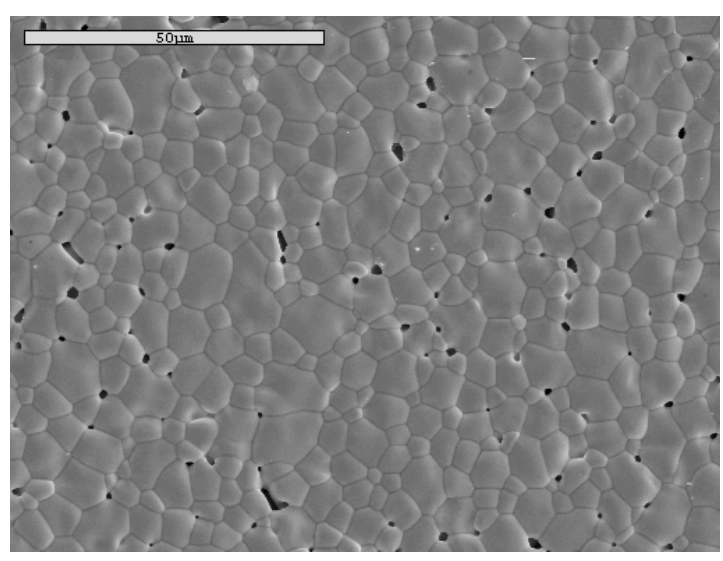

(a) $800^{\circ} \mathrm{C}, 4 \mathrm{hrs} / 1600^{\circ} \mathrm{C}, 6 \mathrm{hrs}$

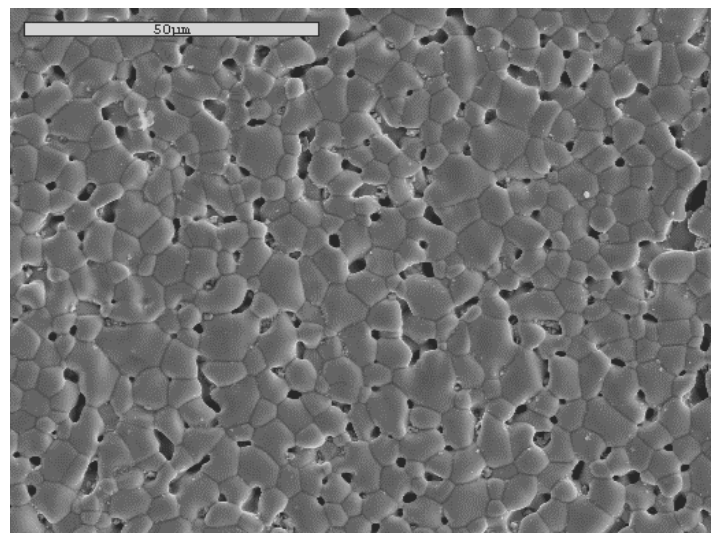

(c) $900^{\circ} \mathrm{C}, 4 \mathrm{hrs} / 1600^{\circ} \mathrm{C}, 6 \mathrm{hrs}$

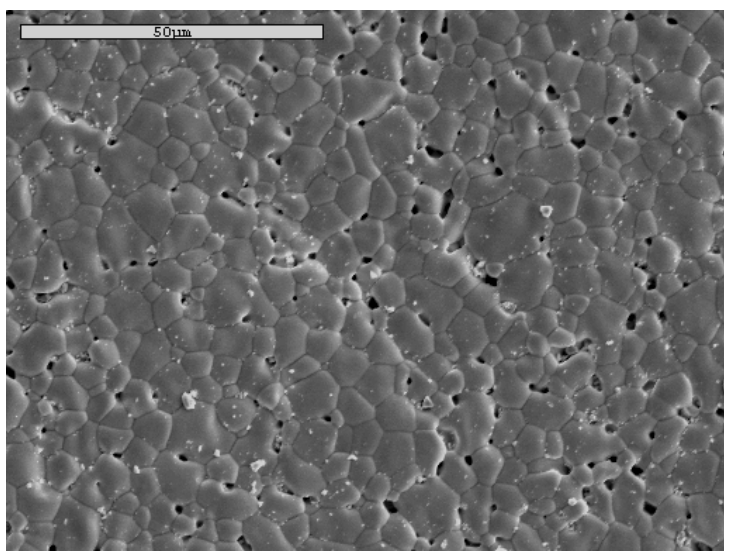

(b) $850^{\circ} \mathrm{C}, 4 \mathrm{hrs} / 1600^{\circ} \mathrm{C}, 6 \mathrm{hrs}$

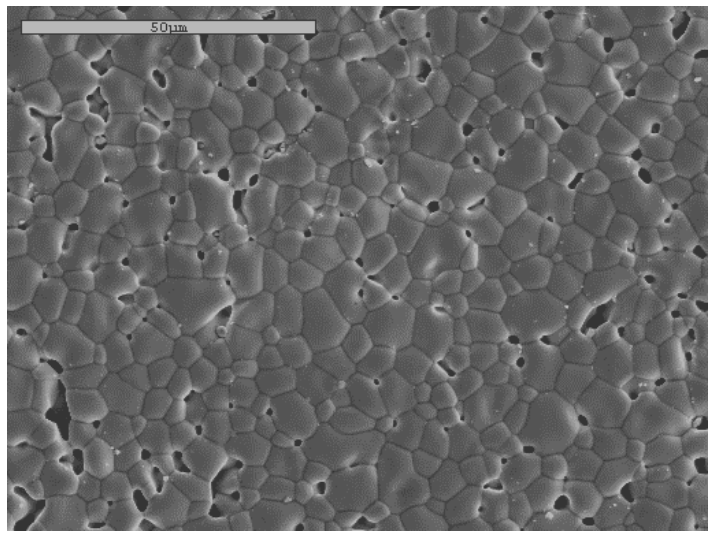

(d) $1000^{\circ} \mathrm{C}, 4 \mathrm{hrs} / 1600^{\circ} \mathrm{C}, 6 \mathrm{hrs}$

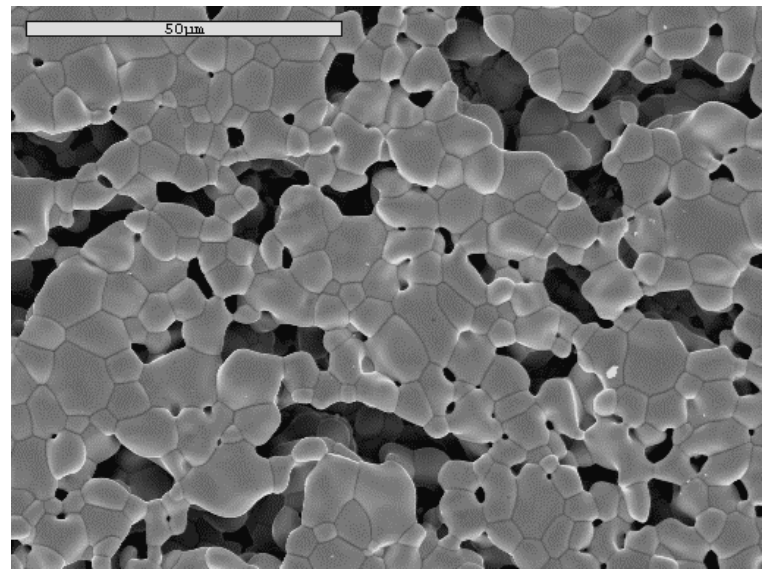

(e) $1100^{\circ} \mathrm{C}, 4 \mathrm{hrs} / 1600^{\circ} \mathrm{C}, 6 \mathrm{hrs}$

Figure 2-13 (a)-(e): Representative microstructures of the Ceria film under different sintering conditions. (Pre-sintering condition /Final Sintering condition) 


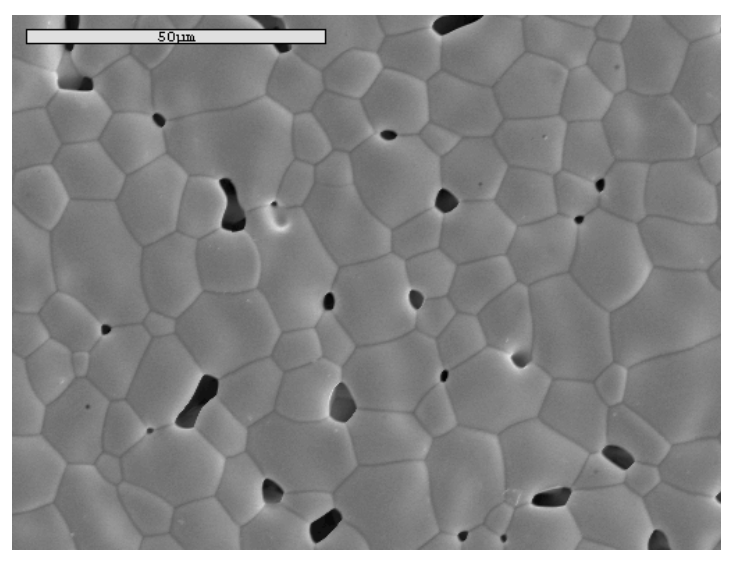

(a) $800^{\circ} \mathrm{C}, 4 \mathrm{hrs} / 1650^{\circ} \mathrm{C}, 10 \mathrm{hrs}$

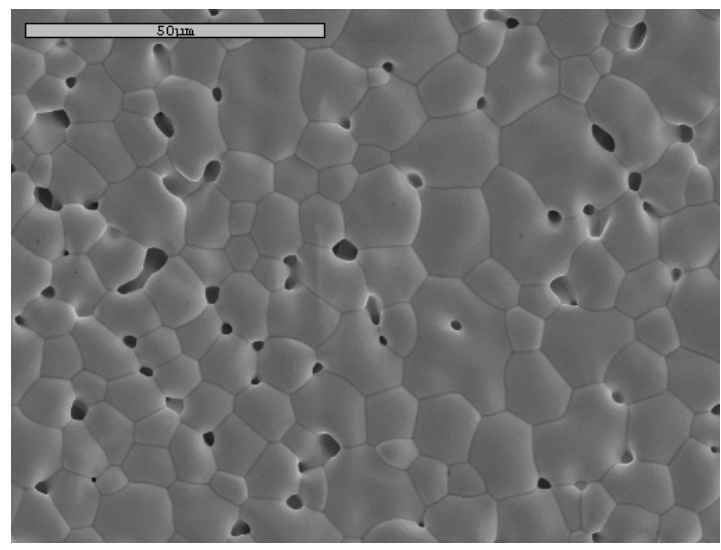

(c) $900^{\circ} \mathrm{C}, 4 \mathrm{hrs} / 1650^{\circ} \mathrm{C}, 10 \mathrm{hrs}$

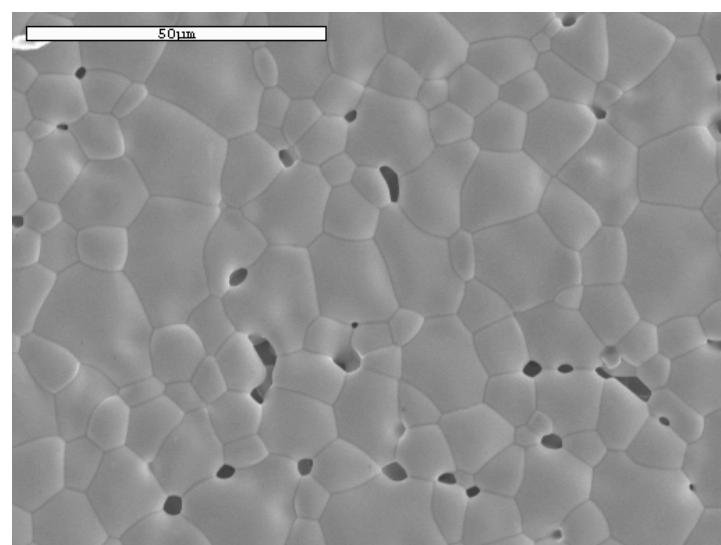

(b) $850^{\circ} \mathrm{C}, 4 \mathrm{hrs} / 1650^{\circ} \mathrm{C}, 10 \mathrm{hrs}$

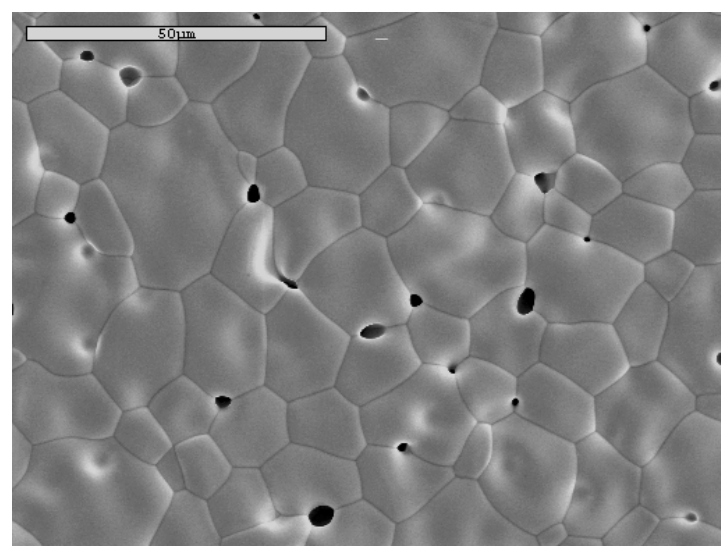

(d) $1000^{\circ} \mathrm{C}, 4 \mathrm{hrs} / 1650^{\circ} \mathrm{C}, 10 \mathrm{hrs}$

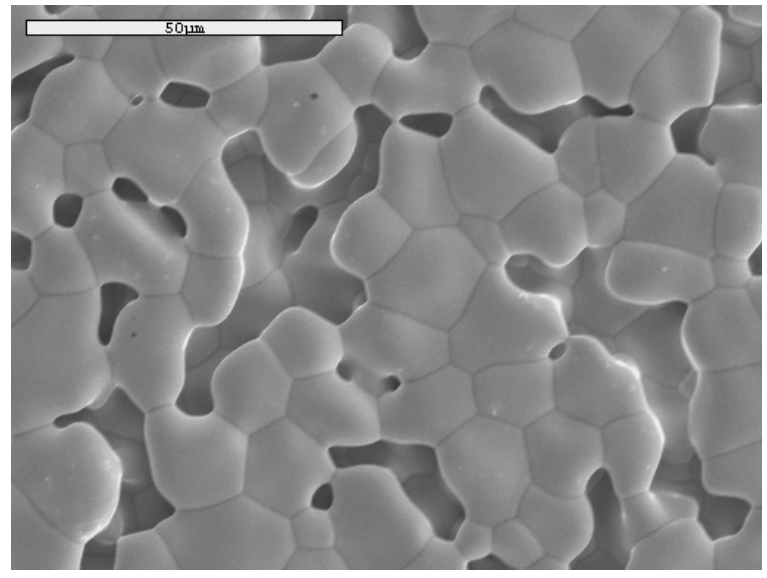

(e) $1100^{\circ} \mathrm{C}, 4 \mathrm{hrs} / 1650^{\circ} \mathrm{C}, 10 \mathrm{hrs}$

Figure 2-14 (a)-(e): Representative microstructures of the Ceria film under different sintering conditions. (Pre-sintering condition/Final Sintering condition). 


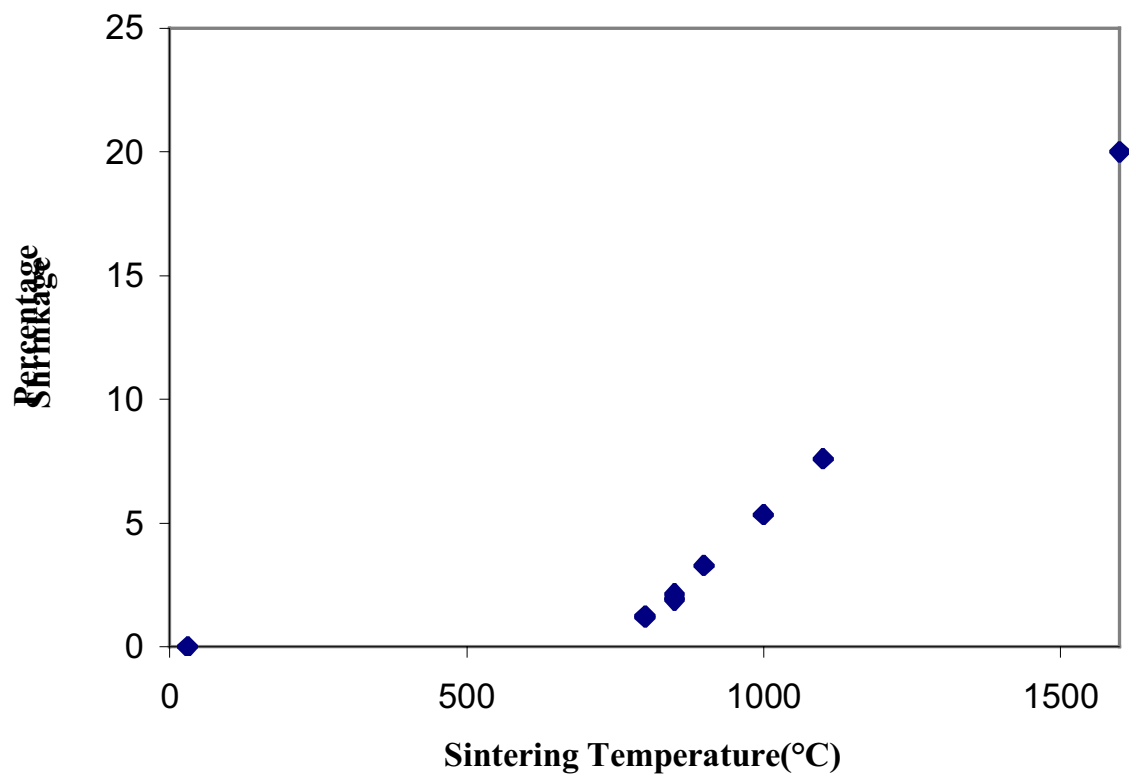

Figure 2-15. Shrinkage profile of the NiO-GDC anode with sintering temperature.

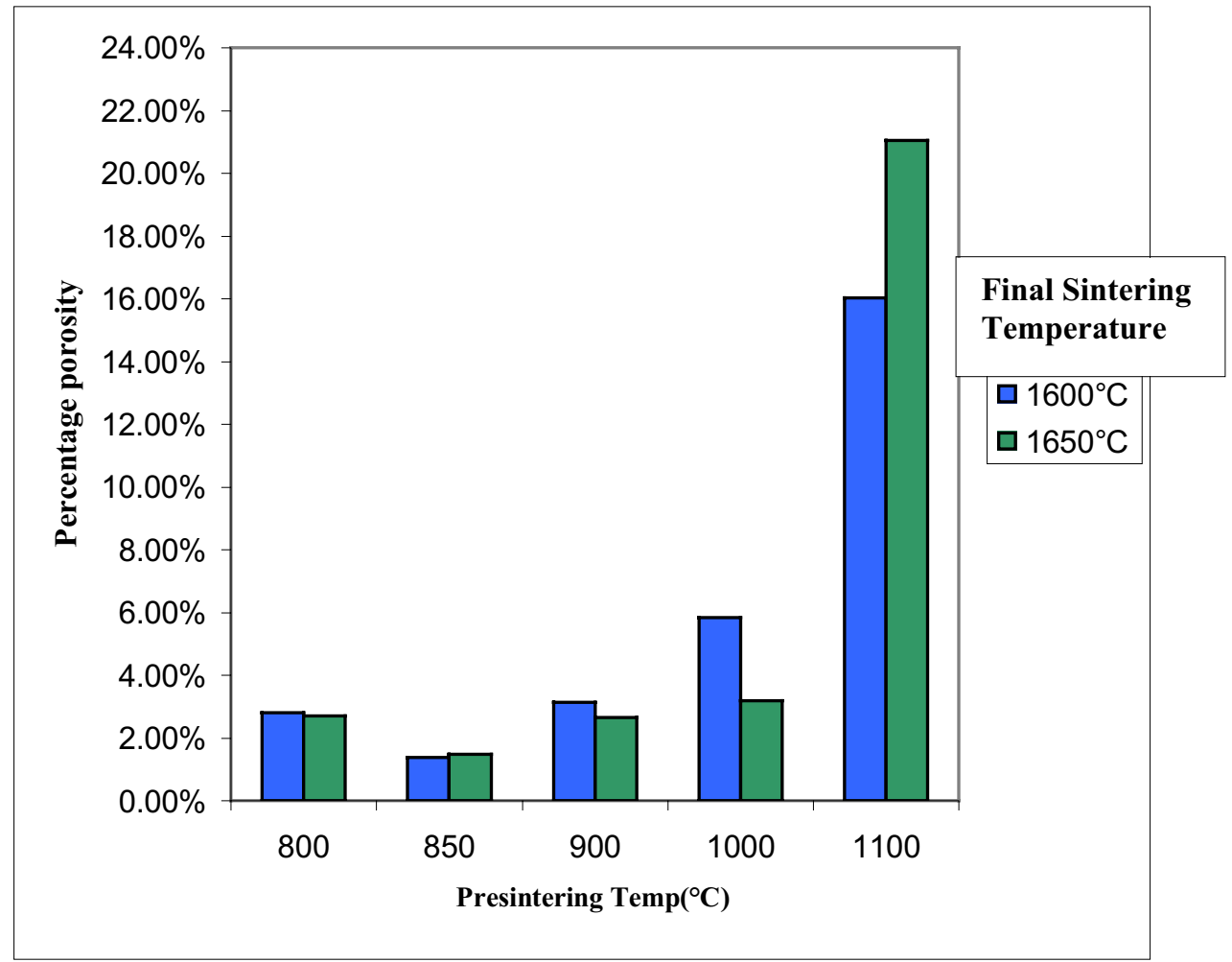

Figure 2-16. Porosity in the Ceria film as a function of the pre-sintering and final sintering temperature of the anode. 


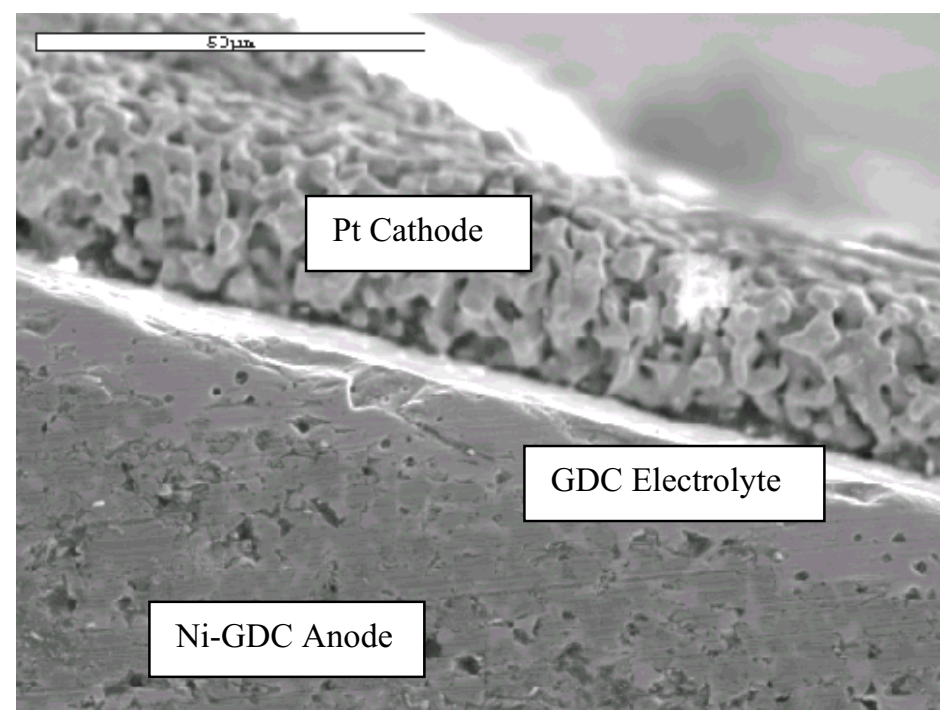

Figure 2-17. Cross-sectional SEM of the film pre-sintered at $850^{\circ} \mathrm{C}$ for 4 hours and finally sintered at $1600^{\circ} \mathrm{C}$ for 6 hours, after being tested in a fuel cell for over 200 hours.

\section{ANALYTICAL MODEL FOR DEFECT DISTRIBUTION AND TRANSPORT IN OPEN-CIRCUIT CONDITIONS}

In this section, the open-circuit electrochemical performance (i.e., the open-circuit potential, $\Phi_{\text {oc }}$ ) of SOFCs is modeled with specific reference to (single layer) SDC-based SOFCs. The model is evaluated by fitting it to the experimental data presented in the next section (section 4). Previous modeling work used a "thermodynamic approach" to develop equations for defect concentration dependence on $P_{\mathrm{O}_{2}}$ (i.e., defect equilibria). Since then a "transport approach" has been developed [36] from the fundamental transport laws and is the method presented below. The approach has proved more versatile in describing transport phenomena and is derived with fewer assumptions than the previous "thermodynamic approach".

To lay the groundwork for deriving the model, the definitions of relevant transport properties and transport laws are given first. Next, the open-circuit spatial concentration distribution of defects in an MIEC in an oxygen potential gradient is derived and expressions for the defect concentration dependence on $P_{\mathrm{O}_{2}}$ are cited from previous work [36]. Expressions for $\Phi_{o c}$ are then derived from the "thermodynamic approach" (based on earlier work [36]) and the "transport approach" (based on the work presented here).

Finally, to close this section, the evaluation of the mass action constant for the external equilibrium reaction, $K_{r}$, and the effect of defect association are explored. The evaluation of this 
important constant is an extension of our previous work [36] and part of an ongoing effort to find methods through which $K_{r}$ may be determined accurately. Secondly, we are interested in the effects defect association may have on the performance of oxide mixed conductors.

\subsection{DEFINITIONS AND LAWS}

The first transport property of interest is the conductivity of the $i$ th species, $\sigma_{i}$, defined as:

$$
\sigma_{i}=z_{i}^{2} q^{2} \frac{D_{i}}{k_{B} T} c_{i}
$$

where $z$ is charge number, $q$ is the elementary charge on an electron, $k_{B}$ is Boltzmann's constant, $T$ is temperature, $D$ is diffusivity and $c$ is concentration. The total conductivity, $\sigma_{t o t}$, of the MIEC is then the sum of all the $i$ th conductivities, written as

$$
\sigma_{t o t}=\sum_{i} \sigma_{i}
$$

A related and useful transport property is the transference number of the $k$ th defect species, $t_{k},-k \in\{1,2, \ldots, i\}$, the set of $i$ defects - which is given by:

$$
t_{k}=\frac{\sigma_{k}}{\sigma_{t o t}}=\frac{\sigma_{k}}{\sum_{i} \sigma_{i}}
$$

The relevant transport laws are given in the next three equations. Firstly, the flux density of the $i$ th species, $j_{i}$ is given by:

$$
j_{i}=-D_{i} \nabla c_{i}-z_{i} D_{i} c_{i} f \nabla \phi=-z_{i}^{-2} q^{-2} \sigma_{i} \nabla \mathbf{f} \mathbf{f}_{i}
$$

where $f=q / k_{B} T$. The electrochemical potential $\mathbf{f}_{i}$ of the $i$ th defect species is given by

$$
\mathbf{f}_{i}=\mu_{i}+z_{i} F \phi=k_{B} T \ln c_{i}+z_{i} q \phi
$$

where $\mu$ is the chemical potential and is defined for, electrically charged, defect species as

$$
\mu_{i}=k_{B} T \ln c_{i}
$$


For, electrically neutral, gaseous species $\mu$ is defined as

$$
\mu_{i}=k_{B} T \ln P_{\mathrm{X}_{\xi}}
$$

where $P_{\mathrm{x}_{\xi}}$ is the partial pressure of the gas X with stoichiometry, in the standard state, $\xi$.

Secondly, the current density, $J$, is the sum of the current density contributions of each $i$ th defect species, $J_{i}$, and given by:

$$
J=\sum_{i} J_{i}=q \sum_{i} z_{i} j_{i}
$$

Finally, the material balance for the $i$ th species may be stated as

$$
\partial c_{i} / \partial t=-\nabla j_{i}+\Gamma_{i}
$$

where $t$ is time and $\Gamma_{i}$, often referred to as production, is the rate of the formation of the $i$ th species due to homogenous chemical reactions in the bulk (i.e., not at the electrode surfaces). It is assumed that the system under consideration is at steady state (i.e., $\partial c_{i} / \partial t=0$ ) and that all chemical reactions take place at the electrode surfaces (i.e., $\Gamma_{i}=0$ ). Thus, Eq. (3.8a) reduces to:

$$
\nabla j_{i}=0
$$

In other words, $j_{i}$ is independent of position.

By definition [37], the open-circuit potential across the fuel cell, $\Phi_{o c}$, is given by

$$
\Phi_{o c}=\frac{1}{2} z_{V}^{-1} q^{-1} \int_{\mu_{0_{20}}}^{\mu_{\mathrm{O}_{2}}} t_{\text {ion }} \cdot \mathrm{d} \mu_{\mathrm{O}_{2}}=\frac{1}{2} z_{V}^{-1} q^{-1} \int_{P_{0}}^{P_{L}} t_{\text {ion }} \cdot \mathrm{d} \ln P
$$

where $P$ is oxygen partial pressure, the subscript " $V$ " refers to oxygen vacancies and the subscripts " 0 " and " $\mathrm{L}$ " refer to conditions on either side of the fuel cell, at $x=0$ and $x=\mathrm{L}$.

Alternatively, if the spatial dependence of the defect species is known, then Eq. (3.9a) may be written as

$$
\Phi_{o c}=\frac{1}{2} z_{V}^{-1} q^{-1} \int_{0}^{L} t_{i o n}\left(\mathrm{~d} \mu_{\mathrm{O}_{2}} / \mathrm{d} x\right) \cdot \mathrm{d} x
$$

where $x$ is the spatial ordinate. 


\subsection{DEFECT DISTRIBUTION IN OPEN-CIRCUIT CONDITIONS}

Defect transport in open-circuit conditions- $J=0, \nabla \phi=0$ and $\nabla c_{i} \neq 0$-is crucially different, see Figure 3-1, from defect transport in closed-circuit conditions- $J \neq 0$ and $\nabla \phi \neq 0$. In closed-circuit conditions the defect mobilities are independent of each other because $J \neq 0$ and there is a net transport of charge across the MIEC. In open-circuit conditions, however, $J=0$ and there is no net transport of charge across the MIEC. The absence of net charge transport implies that the charge carrying species must diffuse simultaneously in a ratio that fulfills the requirement of zero charge transport (i.e., the defect mobilities are no longer independent of each other). This phenomenon is known as ambipolar diffusion.

Therefore, for an MIEC in open-circuit conditions, Eqs. (3.4) and (3.8) reduce to

$$
j_{i}=-D_{a m b i} \nabla c_{i}
$$

and

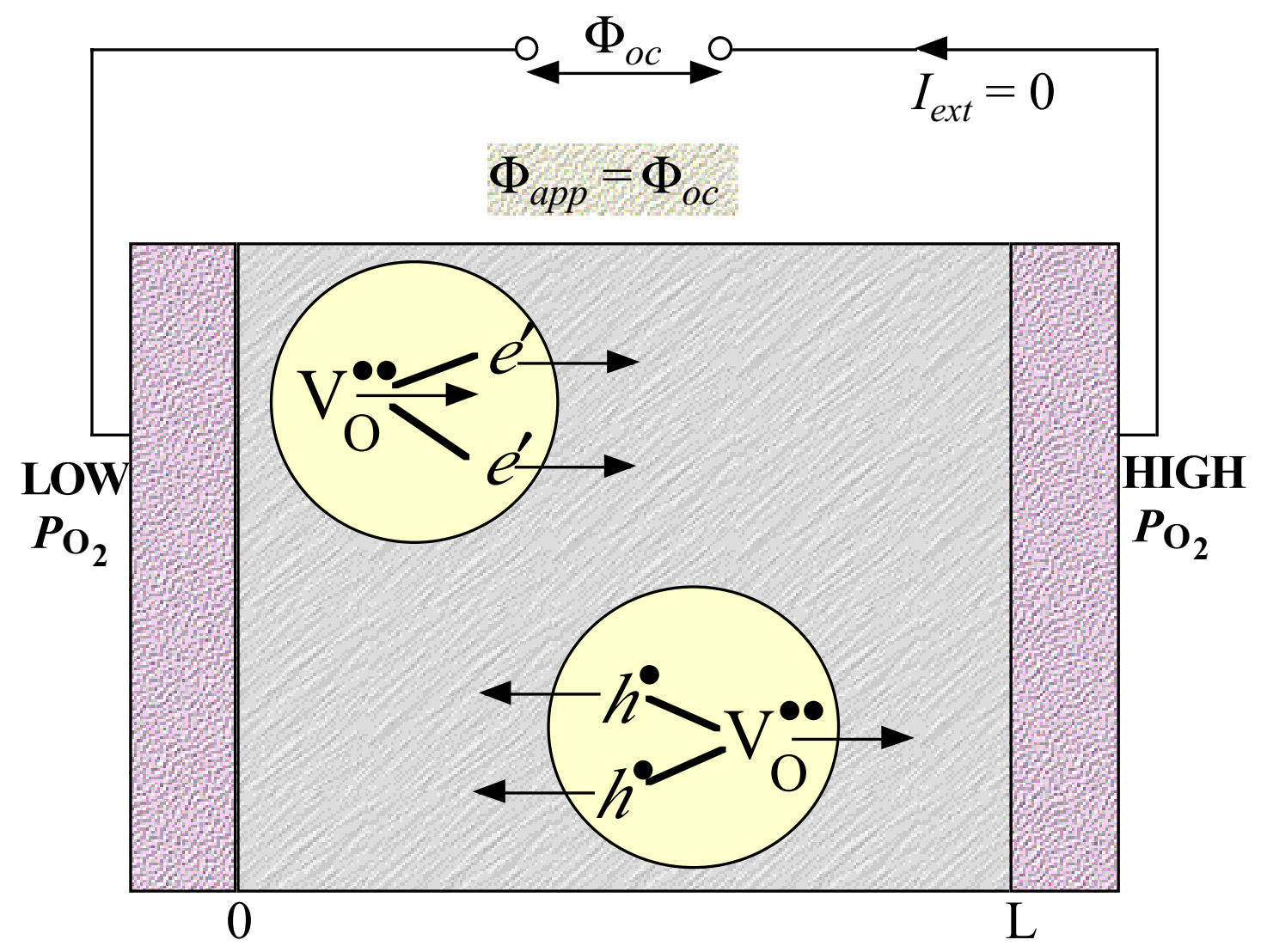

Figure 3-1. Ambipolar defect flux in an SOFC in open-circuit conditions. 


$$
\nabla j_{i}=-D_{a m b i} \nabla^{2} c_{i}=0
$$

Given the usual boundary conditions-at $x=0, c_{i}=c_{i_{0}}$ and at $x=L, c_{i}=c_{i_{\mathrm{L}}}$-then the solution of the second-order differential equation in Eq. (3.11) is

$$
c_{i}(x)=\left(c_{i_{L}}-c_{i_{0}}\right) \frac{x}{L}+c_{i_{0}}
$$

Finally, if the effects of the double layer at the electrode-electrolyte interface may be ignored, then the boundary values of the defect concentrations are set by a combination of the charge balance and the defect equilibrium equation that governs the oxide's equilibrium with the gas phase. The charge balance equation for an acceptor-doped $n$-type oxide MIEC, like samariadoped ceria or gadolinia-doped ceria, is given by

$$
z_{V} c_{V}+z_{e} c_{e}+z_{A} c_{A}=0
$$

where the subscripts " $A$ " and " $e$ " refer to the acceptor dopant and electron respectively. While the oxide-oxygen gas equilibrium reaction is given by

$$
\mathrm{O}_{\mathrm{O}}^{\times} \leftrightarrow \mathrm{V}_{\mathrm{O}}^{\bullet \bullet}+2 e^{\prime}+\frac{1}{2} \mathrm{O}_{2} \quad K_{r}=c_{V} c_{e}^{2} P^{\frac{1}{2}}
$$

where $K_{r}$ is the mass action constant for the external equilibrium reaction. Eqs. (3.13) and (3.14) constitute a pair of simultaneous equations which can be solved (for the defect concentration dependence on oxygen partial pressure) numerically $[38,39]$ or, as we have shown previously [36], through analytical modeling. From our earlier modeling of the defect concentration dependence on oxygen partial pressure in ceria and other MIECs [36], we have:

$$
c_{V}(P)=\left[\frac{3}{4} K_{r}^{\frac{1}{2}} P^{-\frac{1}{4}}+\left(\frac{1}{2} c_{A}\right)^{\frac{3}{2}}\right]^{\frac{2}{3}}
$$

and

$$
c_{e}(P)=K_{r}^{\frac{1}{2}} P^{-\frac{1}{4}}\left[\frac{3}{4} K_{r}^{\frac{1}{2}} P^{-\frac{1}{4}}+\left(\frac{1}{2} c_{A}\right)^{\frac{3}{2}}\right]^{-\frac{1}{3}}
$$


Through substitution of the oxygen partial pressure values at the boundaries $(x=0$ and $x=\mathrm{L})$ of an MIEC in a fuel cell setup (see Figure 3-1), the boundary defect concentrations, $c_{i_{0}}$ and $c_{i_{\mathrm{L}}}$, may be determined from Eqs. (3.15) and (3.16).

\subsection{OPEN-CIRCUIT POTENTIAL}

From the equations developed (and cited) in the two immediately preceding sections, we are now ready to obtain expressions for $\Phi_{o c}$ of an SOFC. There are two approaches that may be used to derive equations for $\Phi_{o c}$. The first combines Eqs. (3.1), (3.2), (3.3), (3.6b), (3.9), (3.15) and (3.16) with the result (since $z_{V}=2$ and $z_{e}=-1$ ):

$$
\begin{aligned}
f \cdot \Phi_{o c} & =\frac{1}{4} \int_{P_{0}}^{P_{L}} \frac{\mathrm{d} \ln P}{1+\Theta K_{r}^{\frac{1}{2}} P^{-\frac{1}{4}}\left[\frac{3}{4} K_{r}^{\frac{1}{2}} P^{-\frac{1}{4}}+\left(\frac{1}{2} c_{A}\right)^{\frac{3}{2}}\right]^{-1}}=\frac{1}{4} \ln \frac{P_{L}}{P_{0}}-\ln \frac{\left(\frac{3}{4}+\Theta\right) K_{r}^{\frac{1}{2}} P_{0}^{-\frac{1}{4}}+\left(\frac{1}{2} c_{A}\right)^{\frac{3}{2}}}{\left(\frac{3}{4}+\Theta\right) K_{r}^{\frac{1}{2}} P_{\mathrm{L}}^{-\frac{1}{4}}+\left(\frac{1}{2} c_{A}\right)^{\frac{3}{2}}} \\
& =f \cdot \Phi_{t h}-\ln \frac{\left(\frac{3}{4}+\Theta\right) K_{r}^{\frac{1}{2}} P_{0}^{-\frac{1}{4}}+\left(\frac{1}{2} c_{A}\right)^{\frac{3}{2}}}{\left(\frac{3}{4}+\Theta\right) K_{r}^{\frac{1}{2}} P_{\mathrm{L}}^{-\frac{1}{4}}+\left(\frac{1}{2} c_{A}\right)^{\frac{3}{2}}}
\end{aligned}
$$

where $\Phi_{t h}$ is the theoretical (Nernst) potential and

$$
\Theta=\frac{z_{e}^{2} D_{e}}{z_{V}^{2} D_{V}}
$$

which is a constant dependent on the ratio of the electron diffusivity, $D_{e}$, to the vacancy diffusivity, $D_{V}$.

An alternative approach combines Eqs. (3.1), (3.2), (3.3), (3.6a), (3.9b), (3.12) and (3.14) with the result

$$
\begin{aligned}
f \cdot \Phi_{o c} & =-\frac{1}{z_{V}} \int_{0}^{L} \frac{c_{V}(x)}{c_{V}(x)+\Theta c_{e}(x)}\left(\nabla \ln c_{V}(x)-\frac{z_{V}}{z_{e}} \nabla \ln c_{e}(x)\right) \cdot \mathrm{d} x \\
& =-\frac{1}{z_{V}}\left\{\frac{1-z_{V}^{2} \Theta}{1-z_{e} z_{V} \Theta} \ln \frac{c_{V_{L}}+\Theta c_{e_{L}}}{c_{V_{0}}+\Theta c_{e_{0}}}-\frac{z_{V}}{z_{e}} \ln \frac{c_{e_{L}}}{c_{e_{0}}}\right\}
\end{aligned}
$$


While both expressions for $\Phi_{o c}$ are useful, the latter result should, in principle, be more accurate since less assumptions were made in it development. Nevertheless, a knowledge of $K_{r}$, $D_{e}$, and $D_{V}$ are critical for the successful application of either equation and is discussed in the following section.

\subsection{EVALUATING THE MAGNITUDE OF THE EQUILIBRIUM CONSTANT, $\boldsymbol{K}_{r}$}

In this section, the evaluation of the external equilibrium constant $K_{r}$ and it relationship with the diffusivity of ionic and electronic defect species is discussed because of their importance in predicting the performance of SOFCs. Ultimately, $K_{r}$ must be obtained from experiments. However, typical conductivity and open-circuit potential experiments measure properties that depend on $K_{r}$ as well as the electron diffusivity, $D_{e}$. Consequently, it is difficult to separate $K_{r}$ and $D_{e}$ from such measurements without independent knowledge of one of them.

Usually, researchers try to circumvent this problem by using other experimental methods such as Hebb-Wagner polarization or thermogravimetry. However, these experiments introduce other problems. Hebb-Wagner polarization experiments, which use blocking electrodes, depend on the reversibility of electrodes used for the experiment $[40,41]$. The assumption of electrode reversibility, however, is often unjustifiable (especially at low temperatures) and becomes poorer as current increases (from zero). Conversely, thermogravimetry is, in principle, an excellent way of determining defect concentration dependence on oxygen partial pressure (from which $K_{r}$ may then be deduced through appropriate methods). However, the small mass variations involved in non-stoichiometry experiments (with oxide MIECs) push the sensitivity of the equipment used to (and often beyond) their limits, making this technique at best very difficult and error prone [40].

In an attempt to address these issues, three methods (including thermogravimetry) of evaluating $K_{r}$ are examined and compared in this section. Two other methods were discussed in an earlier report. As will become increasingly clear (if not already so), $K_{r}$ is a critical constant and it is worth the effort to find reliable ways of determining its value accurately.

\subsubsection{Estimation of $K_{r}$ from Thermogravimetry}

In principle, the best way to determine $K_{r}$ is through thermogravimetric experiments, which are designed to give the weight change of an MIEC sample (and hence the concentration of defects) as a function of $P_{\mathrm{O}_{2}}$ [42]. However, it is usually very difficult to get accurate and reproducible data from thermogravimetric experiments because of the high sensitivity required to detect very small changes in the mass of the specimen [38]. Nevertheless, Kobayashi et al. [43] recently determined the oxygen/metal atom ratios $\left(c_{\mathrm{O}} / c_{c a t}\right)$ for SDC for various temperatures and 
Table 3-1. Calculated values of $K_{r}$ for SDC from thermogravimetry data of Kobayashi et al. [43].

\begin{tabular}{cccc}
\hline $\mathrm{c}_{\mathrm{O}} / \mathrm{c}_{\mathrm{cat}}$ & $\log \mathrm{P}_{\mathrm{O}_{2}}[\ln (\mathrm{atm})]$. & $\mathrm{K}_{\mathrm{r}}\left(\mathrm{m}^{-9} \mathrm{~atm}^{1 / 2}\right)$ & $\ln \mathrm{K}_{\mathrm{r}}\left(\mathrm{m}^{-9} \mathrm{~atm} .^{1 / 2}\right)$ \\
\hline 1.86 & -20.5 & $6.732 \times 10^{71}$ & 165.390 \\
1.87 & -19.5 & $1.149 \times 10^{72}$ & 165.925 \\
1.88 & -18.75 & $1.159 \times 10^{72}$ & 165.934 \\
1.89 & -17.55 & $1.102 \times 10^{72}$ & 165.883 \\
\hline
\end{tabular}

pressures. Oxygen vacancy concentration is related to the $c_{\mathrm{O}} / c_{\text {cat }}$ ratio as follows:

$$
c_{V}=c_{c a t}\left(2-c_{\mathrm{O}} / c_{c a t}\right)
$$

and substituting Eq. (3.20) into Eq. (3.15) and rearranging it yields for $K_{r}$ :

$$
K_{r}^{\frac{1}{2}}=\frac{4}{3} P^{\frac{1}{4}} c_{V}^{\frac{3}{2}}-\frac{4}{3} P^{\frac{1}{4}}\left(\frac{1}{2} c_{A}\right)^{\frac{3}{2}}=\frac{4}{3} P^{\frac{1}{4}}\left[c_{c a t}\left(2-c_{\mathrm{O}} / c_{c a t}\right)\right]^{\frac{3}{2}}-\frac{4}{3} P^{\frac{1}{4}}\left(\frac{1}{2} c_{A}\right)^{\frac{3}{2}}
$$

$K_{r}$ is calculated from Eq. (3.21) for $c_{\mathrm{O}} / c_{\text {cat }}$ ratios in the range 0.186 to 0.189 at $800{ }^{\circ} \mathrm{C}$ where $c_{c a t}=$ $2.5 \times 10^{28} \mathrm{~m}^{-3}$-SDC lattice constant $=0.544 \mathrm{~nm} \mathrm{[44]—and} c_{A}=0.2 c_{\text {cat }}$ using Eq. (3.21); the results are shown in Table 3-1. The values calculated for $K_{r}$ are narrowly distributed and so the standard deviation of the data set for $\ln K_{r}$ is $0.263^{4}\left[\ln \left(\mathrm{m}^{-9} \mathrm{~atm} \cdot{ }^{1 / 2}\right)\right]$, which is $<0.2 \%$ in the worst case. The average value ${ }^{4}$ of $K_{r}$ in Table $3-1$ is $9.97 \times 10^{71} \mathrm{~m}^{-9}$ atm. ${ }^{1 / 2}$.

\subsubsection{Estimation of $K_{r}$ from Open-Circuit Potential}

In this section, expressions derived earlier, Eqs. (3.17) and (3.19), for $\Phi_{o c}$ are used to determine $K_{r}$ from experimental data. Rearranging Eq. (3.17) yields

$$
K_{r}^{\frac{1}{2}}=\frac{\left(\frac{1}{2} c_{A}\right)^{\frac{3}{2}}}{\frac{3}{4}+\Theta} \cdot \frac{1-\left(\frac{P_{L}}{P_{0}}\right)^{-\frac{1}{4}\left(1-\frac{\Phi_{o c}}{\Phi_{t h}}\right)}}{P_{0}^{-\frac{1}{4}}\left(\frac{P_{L}}{P_{0}}\right)^{-\frac{1}{4}\left(1-\frac{\Phi_{o c}}{\Phi_{t h}}\right)}-P_{L}^{-\frac{1}{4}}}
$$

which allows direct calculation of $K_{r}$. Conversely, $K_{r}$ must be calculated from Eq. (3.19) through numerical (e.g., an equation solver) means.

\footnotetext{
4 The geometric mean and variance were required to calculate the standard deviation in the $K_{r}$ values.
} 
In principle, either Eqs. (3.17) or (3.19) allows $K_{r}$ to be determined from measurements of $\Theta$ and $\Phi_{o c}$ for a given $P_{0}$ and $P_{L}$. However, Eq. (3.19) has the advantage of being derived using $c_{i}(x)$, which is an exact solution, Eq. (3.12), of the transport laws (in open-circuit conditions) given in section 4.3. Conversely, Eq. (3.17) is derived using $c_{i}(P)$, Eqs. (3.15) and (3.16), which were modeled using (reasonable) simplifying assumptions. In addition, Eq. (3.19) can be used to determine other external equilibrium constants (i.e., it is not specific to $n$-type fluorites like SDC or GDC) as a function of $\Theta\left(\right.$ or $D_{e}$ ) for given environmental conditions (e.g., $P_{L}, P_{0}, T$, etc.).

In calculating $K_{r}$, experimental values of $\Phi_{o c}$ may need to be corrected for electrode effects, which alter the true oxygen activity seen by the electrolyte at each interface. Furthermore, as Steele [19] points out, values for $D_{e}$, and hence $\Theta$, are difficult to obtain from experiment. Moreover, values available in the literature are inconsistent.

Gödickemeier et al. [45, 46] modeled and evaluated the overpotentials for various electrodes on a SDC electrolyte. From their data for a $\mathrm{La}_{0.84} \mathrm{Sr}_{0.16} \mathrm{CoO}_{3}$ cathode and a Ni$\mathrm{Ce}_{0.9} \mathrm{Ca}_{0.1} \mathrm{O}_{1.9}$ cermet anode on an SDC electrolyte, values of $\Phi_{o c} \approx 800 \mathrm{mV}\left(\Phi_{o c} \approx 740 \mathrm{mV}\right.$ when corrected for electrode overpotentials $[45,46])$ and $\Phi_{t h} \approx 941 \mathrm{mV}$ for $P_{L}=0.21 \mathrm{~atm}$. and $P_{0} \approx 4 \mathrm{x}$ $10^{-19} \mathrm{~atm}$. at $800{ }^{\circ} \mathrm{C}$ were obtained. $K_{r}$ is calculated for various values of $\Theta$, from Eqs. (3.17) and (3.19), using corrected (for electrode overpotential) data from Gödickemeier et al. [45, 46] and the results are shown in Table 3-2.

Table 3-2 shows that, for a given $\Theta \geq 1$, the $K_{r}$ values calculated using Eq. (3.17) or Eq. (3.19) are very close. However, for $\Theta \leq 1$ no unique value of $K_{r}$ is computable from Eq. (3.19). Physically, that is a good result since it implies that, for $\Theta \leq 1$ (i.e., $D_{e} \leq 4 D_{V}$ ), the main reason $\Phi_{o c}<\Phi_{t h}$ (i.e., $\Phi_{o c} / \Phi_{t h}<1$ ) is the presence of the ionic defect concentration gradient. Finally, $K_{r}$ values calculated from Eq. (3.19) are always greater than those from Eq. (3.17). This trend likely indicates that there is an underestimation of the oxygen vacancy concentration gradient in Eq. (3.17), since the difference between the values gets smaller as $\Theta$ increases (i.e., as the effect of $D_{e}$ on $\Phi_{o c}$ becomes more significant than the oxygen vacancy concentration gradient).

Two additional features of Eqs. (3.17) and (3.19) are of particular interest. First, by rewriting Eq. (3.17) as follows:

$$
\frac{K_{r}(3+4 \Theta)^{2}}{16 c_{A}^{3}} \approx \frac{K_{r} \Theta^{2}}{c_{A}^{3}}=\beta=\frac{1}{8}\left[1-\left(\frac{P_{L}}{P_{0}}\right)^{-\frac{1}{4}\left(1-\frac{\Phi_{o c}}{\Phi_{t h}}\right)}\right]^{2}\left[P_{0}^{-\frac{1}{4}}\left(\frac{P_{L}}{P_{0}}\right)^{-\frac{1}{4}\left(1-\frac{\Phi_{o c}}{\Phi_{t h}}\right)}-P_{L}^{-\frac{1}{4}}\right]^{-2}
$$

it becomes evident that for given values of $t^{\prime}{ }_{i o n}, c_{A}, P_{L}$ and $P_{0}$, the term $\beta$ is a constant. 
Table 3-2. Calculated values of $\Theta$ and $K_{r}$ for SDC SOFC from the corrected $\Phi_{o c}$ data of Gödickemeier et al. [45, 46].

\begin{tabular}{ccccc}
\hline \multirow{2}{*}{$\Theta=D_{e} / 4 D_{V}$} & $K_{r}\left(\mathrm{~m}^{-9} \mathrm{~atm}^{1 / 2}\right)$ & $\beta\left(\mathrm{atm}^{1 / 2}\right)$ & $K_{r}\left(\mathrm{~m}^{-9} \mathrm{~atm}^{1 / 2}\right)$ & $\beta\left(\mathrm{atm}^{1 / 2}\right)$ \\
\hline 0.1 & n.a. & n.a. & $8.7 \times 10^{74}$ & $7.0 \times 10^{-11}$ \\
1 & $6.6 \times 10^{75}$ & $5.3 \times 10^{-8}$ & $2.1 \times 10^{74}$ & $1.7 \times 10^{-9}$ \\
10 & $8.1 \times 10^{72}$ & $6.5 \times 10^{-9}$ & $5.5 \times 10^{72}$ & $4.4 \times 10^{-9}$ \\
24.4 & $1.2 \times 10^{72}$ & $5.7 \times 10^{-9}$ & $1 \times 10^{72 \dagger}$ & $4.7 \times 10^{-9}$ \\
26.4 & $1 \times 10^{72 \dagger}$ & $5.6 \times 10^{-9}$ & $8.5 \times 10^{71}$ & $4.7 \times 10^{-9}$ \\
100 & $6.5 \times 10^{70}$ & $5.2 \times 10^{-9}$ & $6.2 \times 10^{70}$ & $5.0 \times 10^{-9}$ \\
1000 & $6.4 \times 10^{68}$ & $5.1 \times 10^{-9}$ & $6.3 \times 10^{68}$ & $5.0 \times 10^{-9}$ \\
\hline
\end{tabular}

$\dagger K_{r}$ calculated from thermogravimetric data of Kobayashi et al. [43], section 4.4.2.

Similarly, for $\Theta c_{e_{0}} \gg c_{V_{0}}, c_{e_{\mathrm{L}}} \ll c_{V_{\mathrm{L}}}=c_{A} / 2$ (typical for SDC or GDC if $P_{\mathrm{L}} \geq \sim 0.21$ atm. and $P_{0} \leq$ $\sim 10^{-18}$ atm. [19, 36, 45 - 47]) and $\Theta \gg 1 / 2$ (i.e., $D_{e} \gg 2 D_{V}$ ), Eq. (3.19) becomes:

$$
f \cdot \Phi_{o c} \approx \ln \frac{c_{V_{L}}}{\Theta c_{e_{L}}}
$$

Moreover, Eqs. (3.13), (3.15) and (3.16) may be substituted into Eq. (3.23a), with the result

$$
\beta=K_{r} \Theta^{2} c_{A}^{-3} \approx \frac{1}{8} P_{\mathrm{L}}^{\frac{1}{2}} \exp \left(f \cdot \Phi_{o c}\right) \equiv \text { constant }
$$

Therefore, from an entirely different approach, Eq. (3.23b) shows that, for a given $\Phi_{o c}, \beta$ is a (material) constant. Thus, one may deduce from Eqs. (3.22) and (3.23b) that, for constant $c_{A}, K_{r}$ is inversely proportional to $\Theta^{2}$. This assertion is borne out in the trends evident in Table 3-2.

A second noteworthy feature of Eqs. (3.22) and (3.23b) is that as $\Phi_{o c} / \Phi_{t h} \rightarrow 1$ (i.e., for good electrolytes) $\beta \rightarrow 0$ (i.e., a very small value). Conversely, as $\Phi_{o c} / \Phi_{t h} \rightarrow 0$ (i.e., for poor electrolytes) Eq. (3.22) $)^{5}$ shows that $\beta \rightarrow \infty$. In other words, the suitability of an oxide MIEC as an electrolyte can be determined from knowledge of $\beta$ which combines information on the reducibility of the MIEC $\left(K_{r}\right)$, the relative diffusivity of the electronic and ionic defects, $\Theta$, and the doping level $\left(c_{A}\right)$.

5 The assumptions made in deriving Eq. (3.19c) from Eq. (3.19) exclude poor electrolytes. Nevertheless, Eq. (3.19c), still shows that $\beta$ attains its maximum value when $\Phi_{O c}=0$. 
Additionally, since $\beta$ is a material constant, it is difficult to separate $K_{r}$ and $\Theta\left(D_{e}\right)$ in experiments which measure properties that are dependent on both defect concentration $\left(K_{r}\right.$ and $\left.c_{A}\right)$ and diffusivity $\left(\Theta\right.$ or $\left.D_{e}\right)$ such as conductivity (next section) and open-circuit potential (discussed in this section) experiments.

Finally, $\ln K_{r}\left(\ln K_{r}=-\Delta G_{r} / k_{B} T\right.$, where $\Delta G_{r}$ is the Gibbs' free energy associated with the reaction in Eq. (3.21) $)^{6}$ doesn't change much even when $\Theta$ varies over $\sim 2$ orders of magnitude. From the preceding discussion, this result may indicate that the oxygen exchange process is limited by the ability of the SDC to produce electrons (i.e., its band gap) rather than the relative speed (i.e., $\Theta$ ) they are supplied to, or consumed from, the interface.

\subsubsection{Estimation of $K_{r}$ from Conductivity Measurements}

$K_{r}$ may also be determined from conductivity measurements. This method is very attractive because electrode effects (typically measured in electrode overpotentials) are essentially absent and thus (in comparison to the preceding strategy) "corrections" (a la Gödickemeier et al. [45, 46]) are unnecessary. Eqs. (3.1), (3.2), (3.15), (3.16) and (2.8) may be combined to yield, for the total conductivity of SDC:

$$
\sigma_{t o t}=z_{V}^{2} f q D_{V}\left(c_{\mathrm{V}}+\Theta c_{e}\right)=q f z_{V}^{2} D_{V}\left\{\left[\frac{3}{4} K_{r}^{\frac{1}{2}} P^{-\frac{1}{4}}+\left(\frac{1}{2} c_{A}\right)^{\frac{3}{2}}\right]^{\frac{2}{3}}+\frac{\Theta K_{r}^{\frac{1}{2}} P^{-\frac{1}{4}}}{\left[\frac{3}{4} K_{r}^{\frac{1}{2}} P^{-\frac{1}{4}}+\left(\frac{1}{2} c_{A}\right)^{\frac{3}{2}}\right]^{\frac{1}{3}}}\right\}
$$

Moreover, if $\frac{3}{4} K_{r}^{\frac{1}{2}} P^{-\frac{1}{4}}<<\left(\frac{1}{2} c_{A}\right)^{\frac{3}{2}}$ then

$$
\sigma_{\text {tot }}=\frac{1}{2} q f z_{V}^{2} D_{V} c_{A}\left\{1+\frac{\Theta K_{r}^{\frac{1}{2}} P^{-\frac{1}{4}}}{\left(\frac{1}{2} c_{A}\right)^{\frac{3}{2}}}\right\}=\frac{1}{2} q f z_{V}^{2} D_{V} c_{A}\left\{1+\beta^{\frac{1}{2}} P^{-\frac{1}{4}}\right\}
$$

By fitting Eq. (3.24a) to conductivity data [47], a unique value of $\Theta$ (or $D_{e}$ ) can be found for any $K_{r}$ or vice versa. In addition, since a data set is fitted rather than one data point (the case for $\Phi_{o c}$ data and Eqs. (3.17) and (3.19)) statistical errors are minimized. The results of this strategy are provided in Table 3-3. In comparison to Table 3-2, Table 3-3 shows higher $K_{r}$ values for each $\Theta$ (or higher $\Theta$ for a given $\mathrm{Kr}$ ) and, accordingly, higher $\beta$ values. Because of the

\footnotetext{
${ }^{6}$ In the strictest sense $K_{r}$ should be dimensionless when used for the calculation of $\Delta G_{r}$. Nonetheless, the analysis presented remains valid since the additional terms required to make $K_{r}$ dimensionless constitute a constant.
} 
Table 3-3. Values of $\Theta, K_{r}$ and $\beta$ calculated from fitting Eq. (3.24) to the SDC conductivity data of Eguchi et al. [47].

\begin{tabular}{cccc}
\hline$\Theta=D_{e} / 4 D_{V}$ & $K_{r}\left(\mathrm{~m}^{-9} \mathrm{~atm} .^{1 / 2}\right)$ & $\ln K_{r}\left(\mathrm{~m}^{-9} \mathrm{~atm}^{1 / 2}\right)$ & $\beta\left(\mathrm{atm}^{1 / 2}\right)$ \\
\hline 0.1 & $1.0 \times 10^{77}$ & 177.3 & $8.0 \times 10^{-9}$ \\
1 & $1.3 \times 10^{76}$ & 175.3 & $1.0 \times 10^{-7}$ \\
10 & $2.0 \times 10^{74}$ & 171.1 & $1.6 \times 10^{-7}$ \\
100 & $2.0 \times 10^{72}$ & 166.5 & $1.6 \times 10^{-7}$ \\
140 & $1.0 \times 10^{72 \dagger}$ & 165.8 & $1.6 \times 10^{-7}$ \\
1000 & $2.0 \times 10^{70}$ & 161.9 & $1.6 \times 10^{-7}$ \\
\hline
\end{tabular}

$\dagger K_{r}$ calculated from thermogravimetric data of Kobayashi et al. [43] in section 4.4.2.

absence of electrode effects and since conductivity measurements are made at equilibrium, the values of $\Theta$ and $K_{r}$ obtained from conductivity measurements (Table 3-3) may be considered to be "more correct" than those from $\Phi_{\mathrm{oc}}$ measurements (Table 3-2). Additionally, the lower $\beta$ values in Table 3-2 may be due to the calculations being made for a single $\left(P_{\mathrm{L}}, P_{0}, T\right)$ coordinate rather than a range as is the case for the conductivity data, in Table 3-3. As will be demonstrated in later sections this can have a significant effect on the calculations.

In closing this section, it is worth noting that the $\Delta G_{r}$ values (i.e., $R T \ln K_{r}$ ) calculated (for a given $\Theta$ ) from any of the electrical/electrochemical methods-Eqs. (3.17), (3.19) or (3.24) — are, given acceptable experimental error, almost the same. This speaks to the utility and correctness of the strategies employed to calculate $\Delta G_{r}\left(\right.$ or $\left.K_{r}\right)$.

\section{OPEN CIRCUIT POTENTIAL AND MODEL VERIFICATION FOR SDC SOFCS}

In this section, the electrochemical performance of SOFCs assembled in our laboratory consisting of SDC electrolytes and porous platinum $(\mathrm{Pt})$ or gold $(\mathrm{Au})$ electrodes with air on the cathode side of the electrolyte and either $\mathrm{CO} / \mathrm{CO}_{2}$ or $\mathrm{H}_{2} / \mathrm{H}_{2} \mathrm{O}$ gas mixture at the anode are reported (sections 4.1 and 4.2). The model developed in section 3-Eq. (3.19)-is then evaluated by fitting it to the experimental data presented in section 4.1 .

\subsection{OPEN CIRCUIT POTENTIAL}

Porous Pt electrodes were applied to samples with density $\geq 90 \%$ of the theoretical density. These samples were used to construct an SOFC of the type;

$$
P_{\mathrm{O}_{2}}, \mathrm{Pt} / \mathrm{SDC} / \mathrm{Pt}, P_{\mathrm{CO} / C O_{2}}\left(\text { or } P_{\mathrm{H}_{2} / \mathrm{H}_{2} \mathrm{O}}\right)
$$




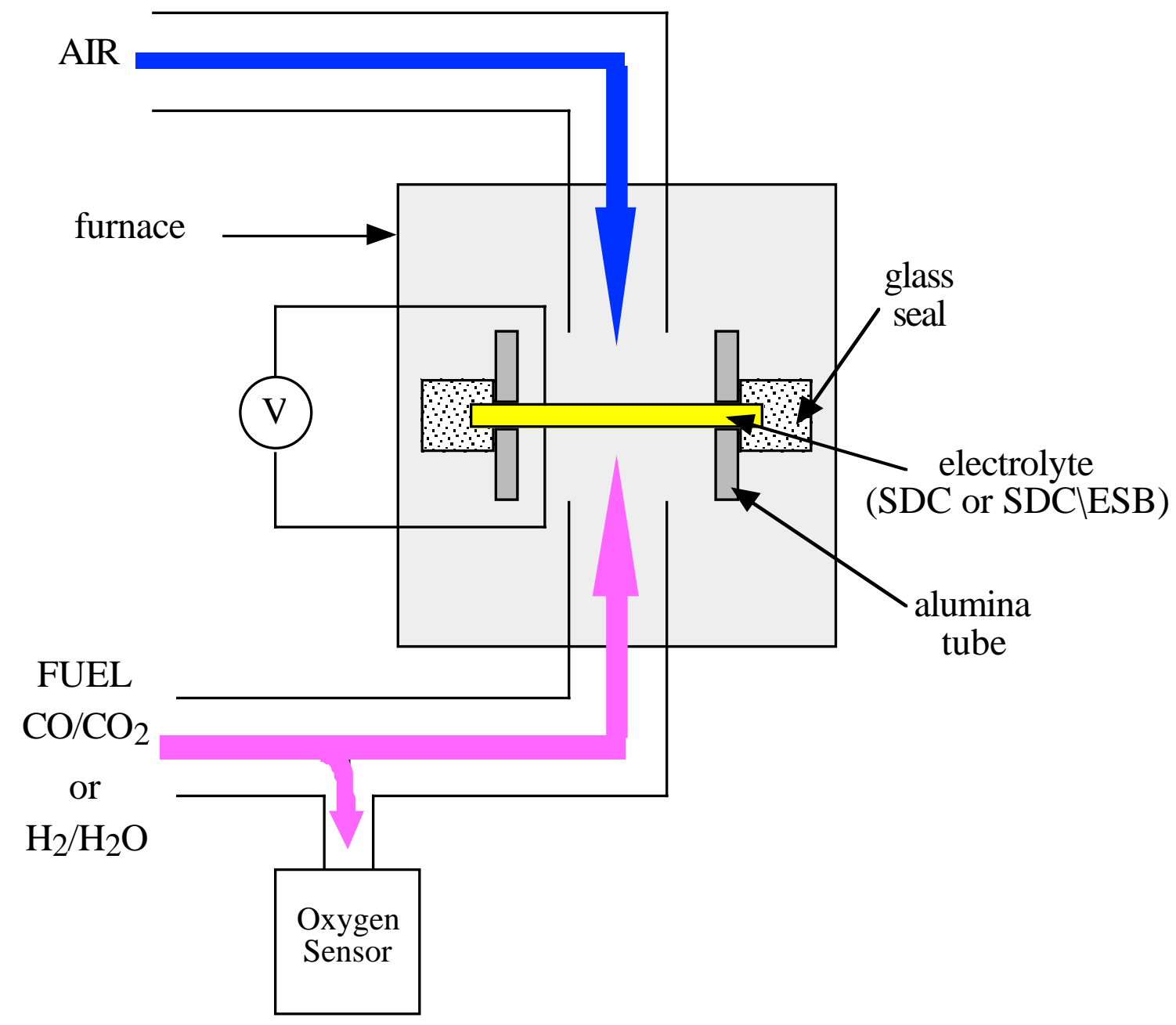

Figure 4-1. Setup of the fuel cell apparatus.

with air as the oxidizing gas and $\mathrm{CO} / \mathrm{CO}_{2}$ or $\mathrm{H}_{2} / \mathrm{H}_{2} \mathrm{O}$ gas mixtures as the reducing gas. $\Phi_{o c}$ was then measured with a Keithley 2000 multimeter, as shown in Figure 4-1, for various $P_{\mathrm{O}_{2}}$ 's and temperatures and used to calculate the apparent transference number, $t^{\prime}{ }_{i o n}$, which is given by

$$
t_{i o n}^{\prime}=\frac{\Phi_{o c}}{\Phi_{t h}}
$$

where $\Phi_{t h}$ is the theoretical (Nernst) potential.

Each $P_{\mathrm{O}_{2}}$ was set by the mixing ratio of the reducing gases (the $P_{\mathrm{O}_{2}}$ on the oxidizing side was kept constant). The $P_{\mathrm{O}_{2}}$ of each reducing gas mixture was measured with a zirconia (automotive) oxygen sensor, placed in series with the SOFC, with (stagnant) air on the reference 
side and the reducing gas combination to be measured on the sensing side. The sensor potential is taken as equal to the theoretical (Nernst) potential, $\Phi_{t h}$, for the fuel cell since $t_{i o n}^{\prime}=1$ for zirconia. Therefore:

$$
\Phi_{\text {sensor }}=\Phi_{t h}=\frac{k_{B} T}{4 q} \ln \frac{P_{L}}{P_{0}}
$$

where $P_{\mathrm{L}}$ is the $P_{\mathrm{O}_{2}}$ on the oxidizing/air side (at $x=\mathrm{L}$ ) and $P_{0}$ is the $P_{\mathrm{O}_{2}}$ on the reducing/fuel side (at $x=0) . \quad P_{0}$ may then be calculated from a knowledge of the sensor potential, $P_{\mathrm{L}}$ and temperature. Additional details of the experimental procedure may be obtained elsewhere [48].

Figures 4-2 and 4-3 show the dependence of $\Phi_{o c}$ and $t_{i o n}^{\prime}$ on $P_{\mathrm{O}_{2}}$ in the temperature range $500{ }^{\circ} \mathrm{C}$ to $800{ }^{\circ} \mathrm{C}$. In Figure $4-3$, the experimental error (calculated statistically) in $t^{\prime}{ }_{i o n}$, arising primarily from small, random fluctuations, are represented as error bars. In Figure 4-2, $\Phi_{o c}$ (see Eq. (4.1)) is near theoretical (sensor) for high $P_{\mathrm{O}_{2}}$ and therefore falls on a $45^{\circ}$ line. At low $P_{\mathrm{O}_{2}}$, $\Phi_{o c}$ begins to deviate due to the onset of electronic conduction. Correspondingly, in Figure 4-3, $t_{i o n}^{\prime}$ was constant and close to unity in the high $P_{\mathrm{O}_{2}}$ region and decreased in the low $P_{\mathrm{O}_{2}}$ region. The $P_{\mathrm{O}_{2}}$ for the onset of electronic conduction (i.e., $t_{i o n}^{\prime} \leq 0.9$ for $P_{\mathrm{O}_{2}} \leq P_{\mathrm{O}_{2}}^{\text {onset }}$ ) is shown in Table 4-1. As expected, the $\Phi_{o c}$ and $t^{\prime}{ }_{i o n}$ of the SOFCs decreased with increasing temperature as a result of the diminishing ionic domain of the SDC electrolyte. In Figure 4.2a, $\Phi_{o c}$ is normalized by the factor $\mathrm{nF} / \mathrm{RT}$ in order to make the curves more visually distinguishable. 


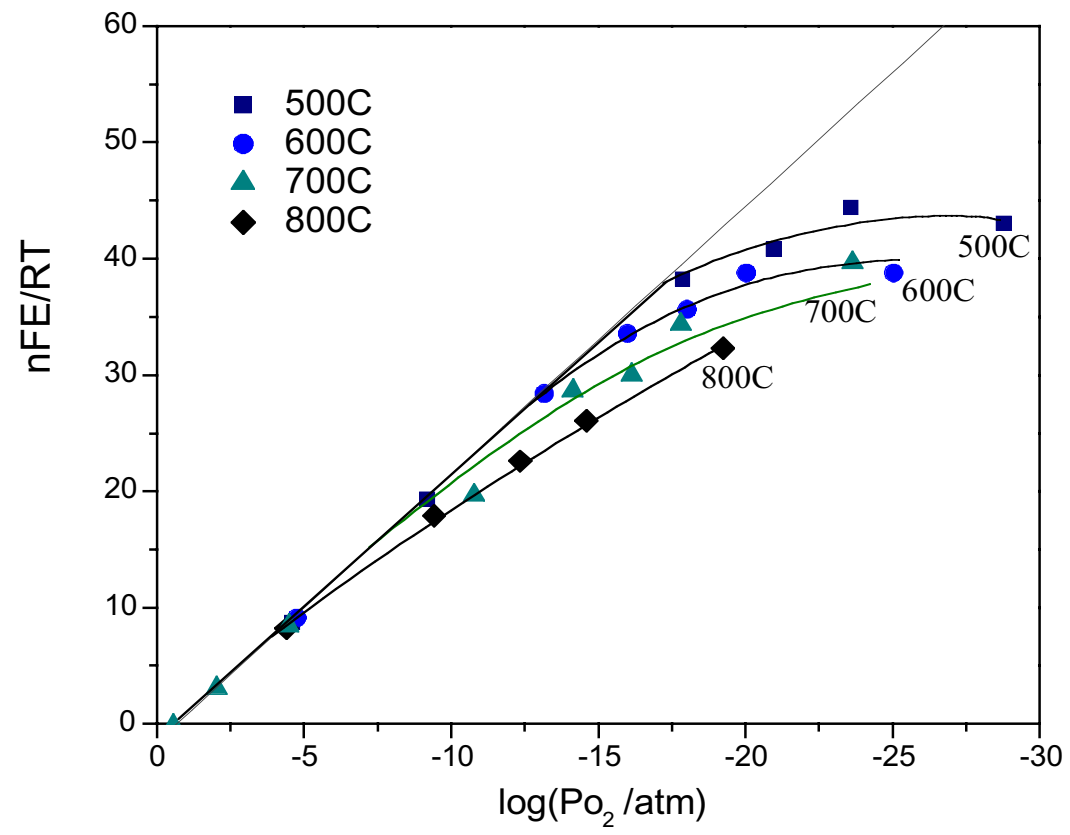

(a)

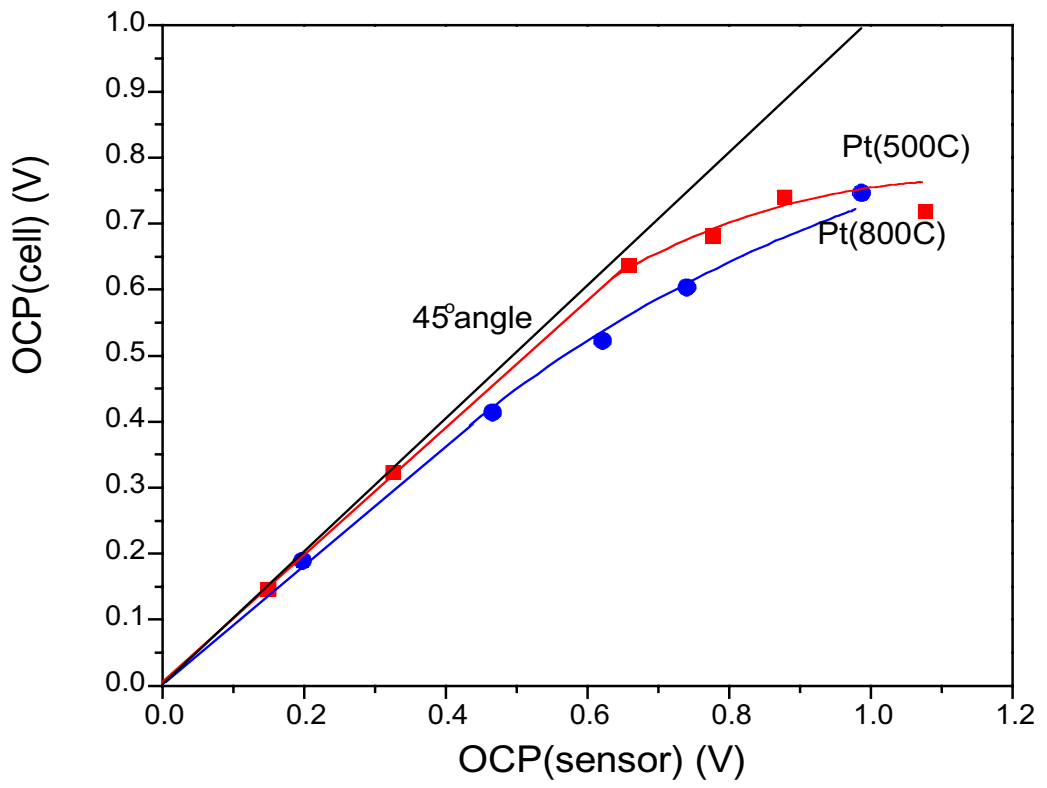

(b)

Figure 4-2. Normalized OCP $(n F E / R T)$ of the SOFC, $P_{\mathrm{O}_{2}}, \mathrm{Pt} / \mathrm{SDC} / \mathrm{Pt}, P_{{\mathrm{CO} / \mathrm{CO}_{2}}}\left(\right.$ or $\left.P_{\mathrm{H}_{2} / \mathrm{H}_{2} \mathrm{O}}\right)$, as a function of (a) $P_{\mathrm{O}_{2}}$ and (b) sensor potential $\left(\Phi_{t h}\right)$. 


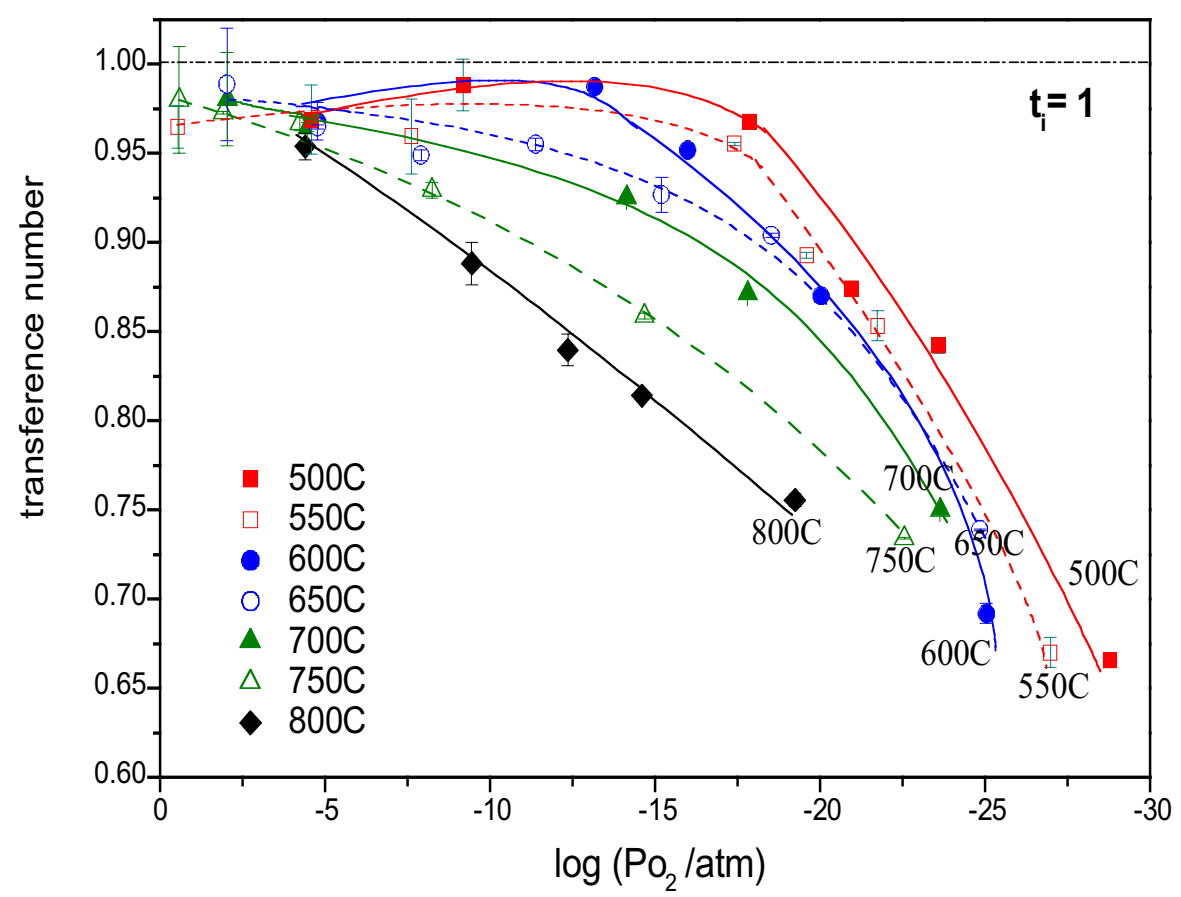

Figure 4-3. Dependence of $t^{\prime}$ ion on $P_{\mathrm{O}_{2}}$ for $500{ }^{\circ} \mathrm{C} \leq T \leq 800{ }^{\circ} \mathrm{C}$ for the SOFC, $P_{\mathrm{O}_{2}}, \mathrm{Pt} / \mathrm{SDC} / \mathrm{Pt}$, $P_{\mathrm{CO} / \mathrm{CO}_{2}}\left(\right.$ or $\left.P_{\mathrm{H}_{2} / \mathrm{H}_{2} \mathrm{O}}\right)$.

\subsection{EFFECT OF CATHODE MATERIAL (Au AND Pt)}

The electrochemical performance of SOFCs is influenced greatly by the characteristics of the electrodes. In this work, both $\mathrm{Pt}$ and $\mathrm{Au}$ electrodes were used in the ceria-based SOFCs. Au is a notoriously bad electrode material. However, it is a stable (i.e., it does not react with ESB) material for use as a cathode on the SDCLESB bilayers. To evaluate the performance of the $\mathrm{Au}$ electrode on SDC electrolytes (and later for comparison with bilayered SDCLESB electrolytes) an SOFC was constructed of the following type

$$
\mathrm{P}_{\mathrm{O}_{2}}, \mathrm{Au} / \mathrm{SDC} / \mathrm{Pt}, P_{\mathrm{CO} / \mathrm{CO}_{2}}\left(\text { or } P_{\mathrm{H}_{2} / \mathrm{H}_{2} \mathrm{O}}\right)
$$

and its $\Phi_{o c}$ values compared with those obtained from the SOFC in Eq. (4.1).

Figure 4-4 shows the $P_{\mathrm{O}_{2}}$ dependence of $\Phi_{o c}$ in the temperature range: $650{ }^{\circ} \mathrm{C} \leq \mathrm{T} \leq 800$ ${ }^{\circ} \mathrm{C}$ and Figures 4-5 and 4-6 show the $P_{\mathrm{O}_{2}}$ dependence of $t^{\prime}$ ion in the temperature range: $500{ }^{\circ} \mathrm{C} \leq \mathrm{T}$ $\leq 800{ }^{\circ} \mathrm{C}$ for the SDC SOFC with the Au cathode. Table 4-1 compares the values of $P_{\mathrm{O}_{2}}$ at which $t_{i o n}^{\prime}$ drops below 0.9 for SDC SOFCs with Pt and Au cathodes. Godickemeier et al.[45, 


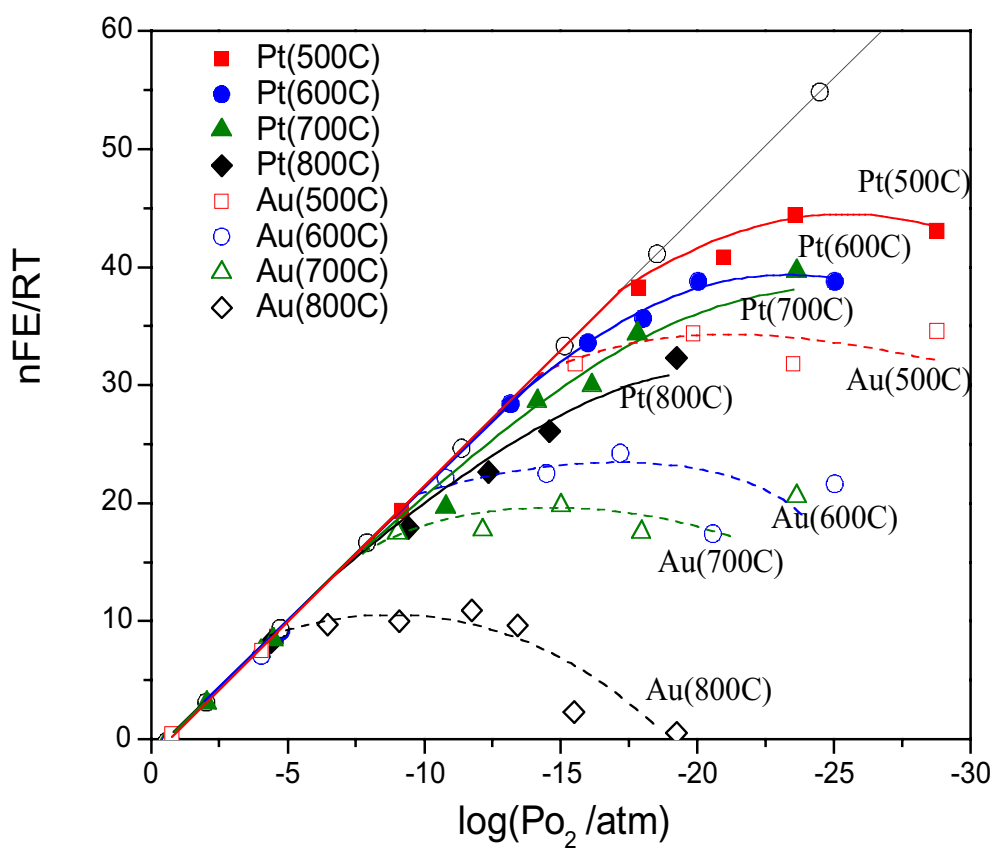

(a)

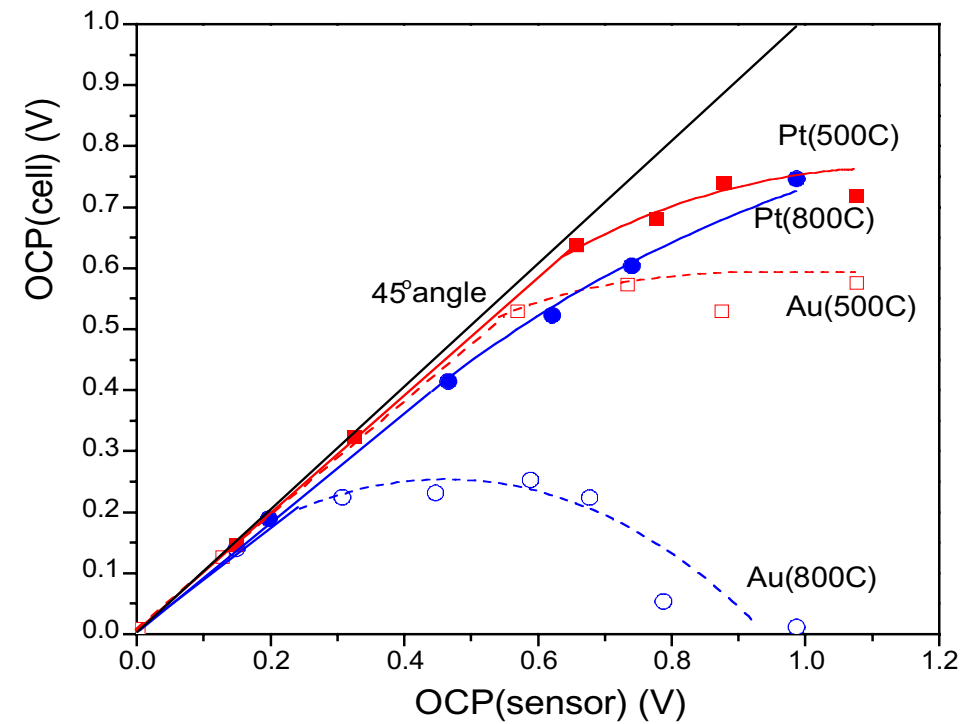

(b)

Figure 4-4. Comparison of normalized OCP (nFE/RT) of SOFCs in Eqs. (4.1) and (4.4) as a function of (a) $P_{\mathrm{O}_{2}}$ and (b) sensor potential $\left(\Phi_{t h}\right)$. 


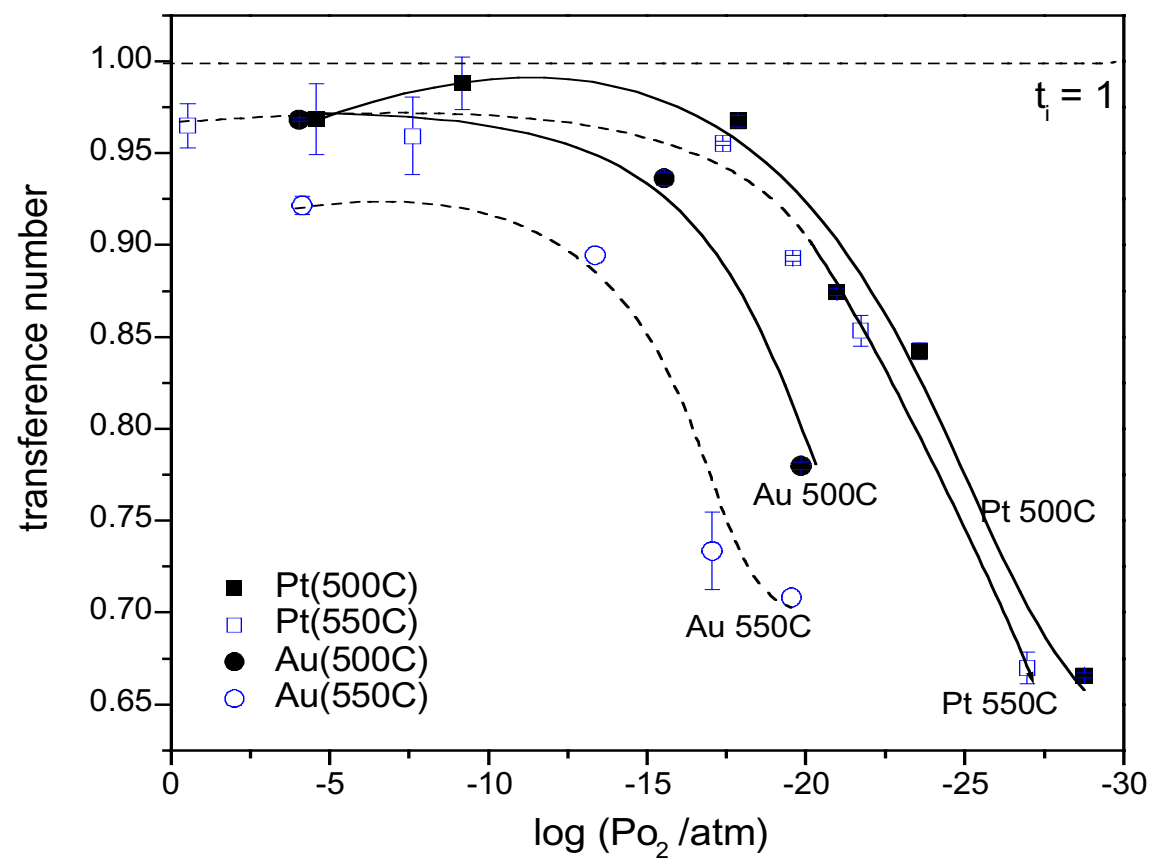

(a)

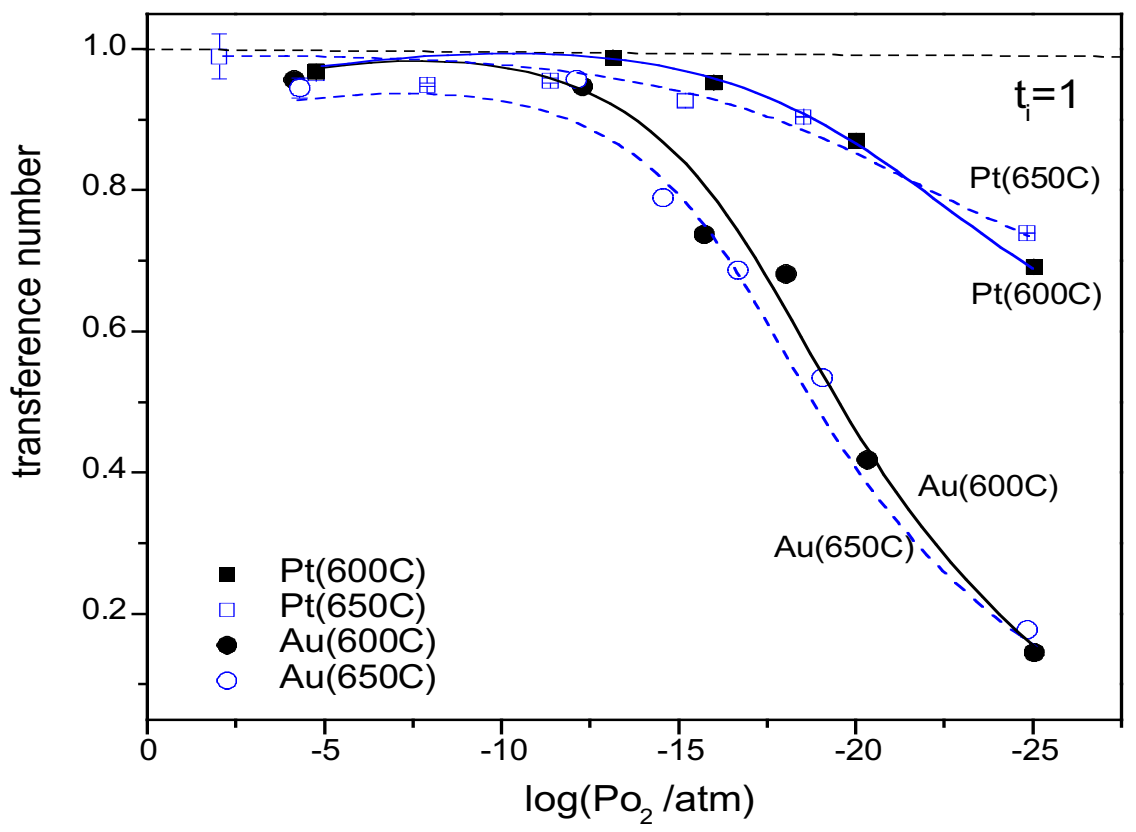

(b)

Figure 4-5. Comparison of $t_{i o n}^{\prime}$ for SOFCs in Eqs. (4.1) and (4.4) as a function of $P_{\mathrm{O}_{2}}$ at (a) 500 and $550^{\circ} \mathrm{C}$ and (b) 600 and $650^{\circ} \mathrm{C}$. 


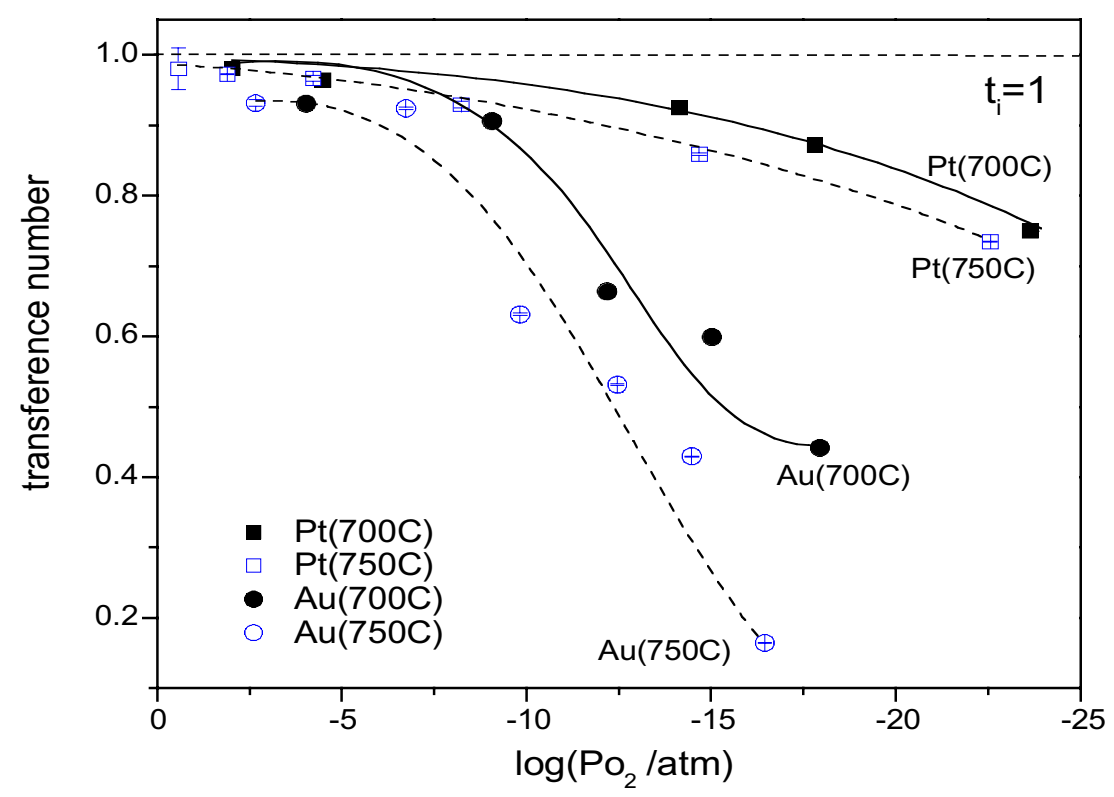

(a)

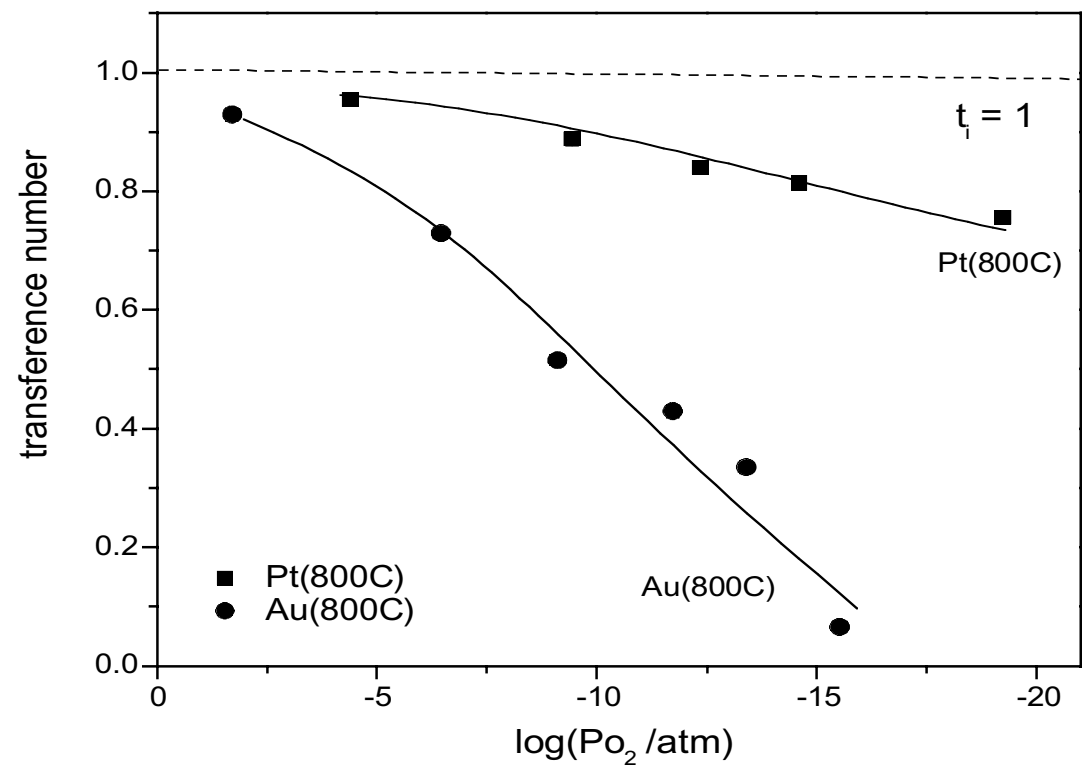

(b)

Figure 4-6. Comparison of $t_{i o n}^{\prime}$ for SOFCs in Eqs. (4.1) and (4.4) as a function of $P_{\mathrm{O}_{2}}$ at (a) 700 and 750rC and (b) 800rC. 
Table 4-1. Oxygen Partial Pressure at which $t_{i o n}^{\prime}=0.90$ for SOFCs in Eqs. (4.1) and (4.4).

\begin{tabular}{ccc}
\hline Temperatures (rC) & $P_{\mathrm{O}_{2}}$, Pt cathode (atm.) & $P_{\mathrm{O}_{2}}$, Au cathode (atm.) \\
\hline 500 & $\sim 10^{-22}$ & $\sim 10^{-17.5}$ \\
550 & $\sim 10^{-21}$ & $\sim 10^{-14}$ \\
600 & $\sim 10^{-19.5}$ & $\sim 10^{-13.5}$ \\
650 & $\sim 10^{-18}$ & $\sim 10^{-13}$ \\
700 & $\sim 10^{-16}$ & $\sim 10^{-9.5}$ \\
750 & $\sim 10^{-12}$ & $\sim 10^{-7.5}$ \\
800 & $\sim 10^{-8}$ & $\sim 10^{-4}$ \\
\hline
\end{tabular}

46] showed that both $\mathrm{Au}$ and Pt electrodes allow for oxygen transport with Au cathodes exhibiting a higher electrode overpotential than Pt cathodes. This is a consequence of Au being a poor electro-catalyst. Since $\mathrm{Pt}$ is electro-catalytically more active than $\mathrm{Au}$, the chemical potential difference $\left(\mu_{\mathrm{O}_{2}}^{\prime \prime}-\mu_{\mathrm{O}_{2}}^{\prime}\right)$ across the electrolyte of a concentration cell with Pt electrodes is greater than with Au electrodes; this is shown schematically in Figure 4-7. Accordingly, a higher $\Phi_{o c}$ (and $t^{\prime}{ }_{i o n}$ ) was obtained for the SDC SOFC that had a Pt rather than a Au cathode.

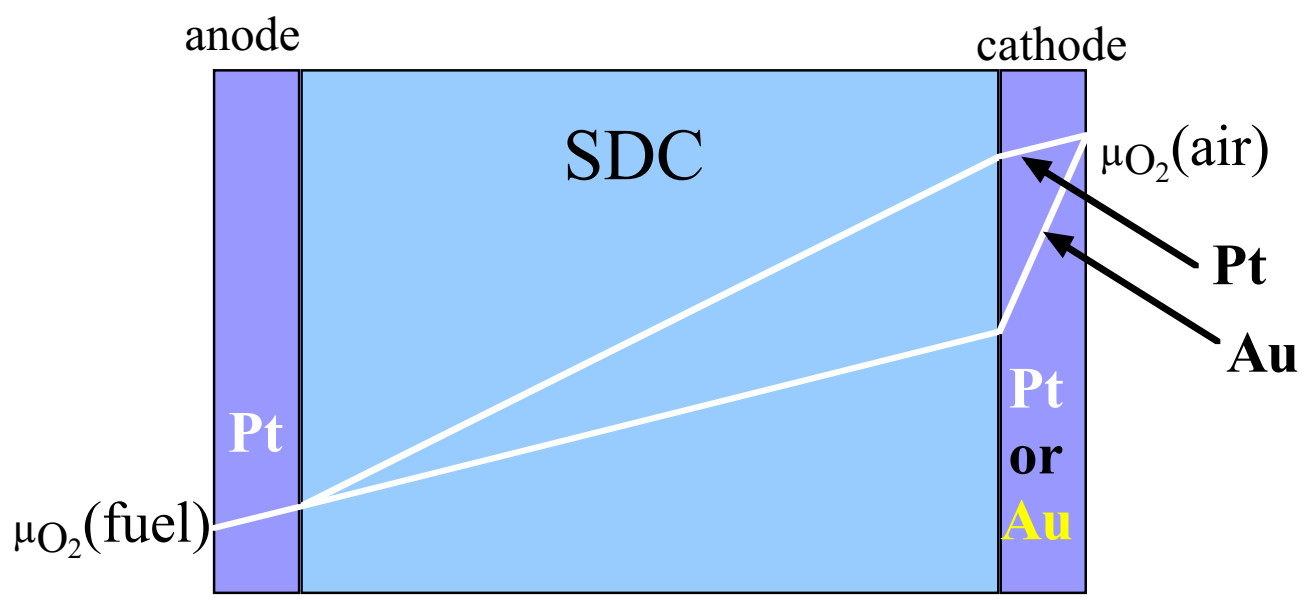

Figure 4-7. Schematic representation comparing the effect of electrode overpotential on the variation of $\mu_{\mathrm{O}_{2}}$ across a SOFC with an Au vs. a Pt cathode. 


\subsection{EXPERIMENTAL VERIFICATION OF THE MODEL}

In order to verify the accuracy and validity the model developed for open-circuit conditions in section 3.3, Eq. (3.19) is fitted to the experimental data in Figures 4-8 through 414. This was done by adjusting the parameters $K_{r}$ and $\Theta$ (i.e., $D_{e} / D_{V}$ ) to maximize the correlation coefficient, $r$, (and $\breve{r}$, the corrected ${ }^{7}$ correlation coefficient) [49] between the data and the model (i.e., to obtain the best fit of the model to the data). Table 4-2 shows the parameters used for each plot and the correlation coefficients obtained. The high correlation coefficients obtained and the fact that only two degrees of freedom are allowed are testament to the ability of the model to describe the physics relating the material properties of the electrolyte material $\left(K_{r}\right.$ and $\Theta)$ to the electrochemical performance of the SOFC.

Figures 4-8 through 4-14 show that as $P_{0}$ decreases below a critical value, $\Phi_{\mathrm{oc}}$ becomes less than $\Phi_{t h}$. This corresponds to an increase in the electronic conductivity of the electrolyte, which is attributable to the increasing concentration of electrons on the reducing side of the electrolyte due to the decreasing $P_{\mathrm{O}_{2}}$. In addition, the deviation of the $\Phi_{\mathrm{oc}}$ from $\Phi_{\text {th }}$ starts progressively later as the temperature decreases which corresponds to a widening of the electrolytic domain of SDC, a phenomenon reported by other researchers. At $800{ }^{\circ} \mathrm{C}, \Phi_{\mathrm{oc}}$ starts to deviate from $\Phi_{\text {th }}$ at $\sim 0.3 \mathrm{~V}$ while at $500{ }^{\circ} \mathrm{C}$ the deviation begins at $\sim 0.7 \mathrm{~V}$. This behaviour is a consequence of the exponential dependence of $K_{r}$ on reciprocal temperature (1/T). Thus, $K_{r}$ increases and SDC produces electrons more readily (i.e., at a higher $P_{\mathrm{O}_{2}}$ ) as the operating temperature increases (see Eq. (3.19)).

Another noteworthy feature of Figures 4-8 to 4-14, is that as the temperature decreases, the shape of the curve for $\Phi_{\mathrm{oc}}$ becomes more plateau-like once the deviation from $\Phi_{\mathrm{th}}$ begins. This phenomenon may be explained by considering that at low temperatures $D_{e} / D_{V} \gg 1$ and $c_{V_{\mathrm{L}}}$ $\gg>c_{e_{\mathrm{L}}}$, under these conditions Eq. (3.19) reduces to:

$$
\Phi_{o c}=-\frac{k_{B} T}{z_{e} q} \ln \frac{c_{V_{L}}+\Theta c_{e_{L}}}{\Theta c_{e_{L}}}
$$

In other words, at low temperatures $\Phi_{o c}$ is independent of $P_{0}$, a circumstance predicted by the model and borne out by experiment.

\footnotetext{
${ }^{7}$ In fitting Eq. (3.19) to experimental data, fitting parameters, $K_{r}$ and $\Theta$, are adjusted, i.e., the model has two degrees of freedom. The correlation coefficient, $r$, is often corrected to reflect the presence of the fitting parameters [49].
} 


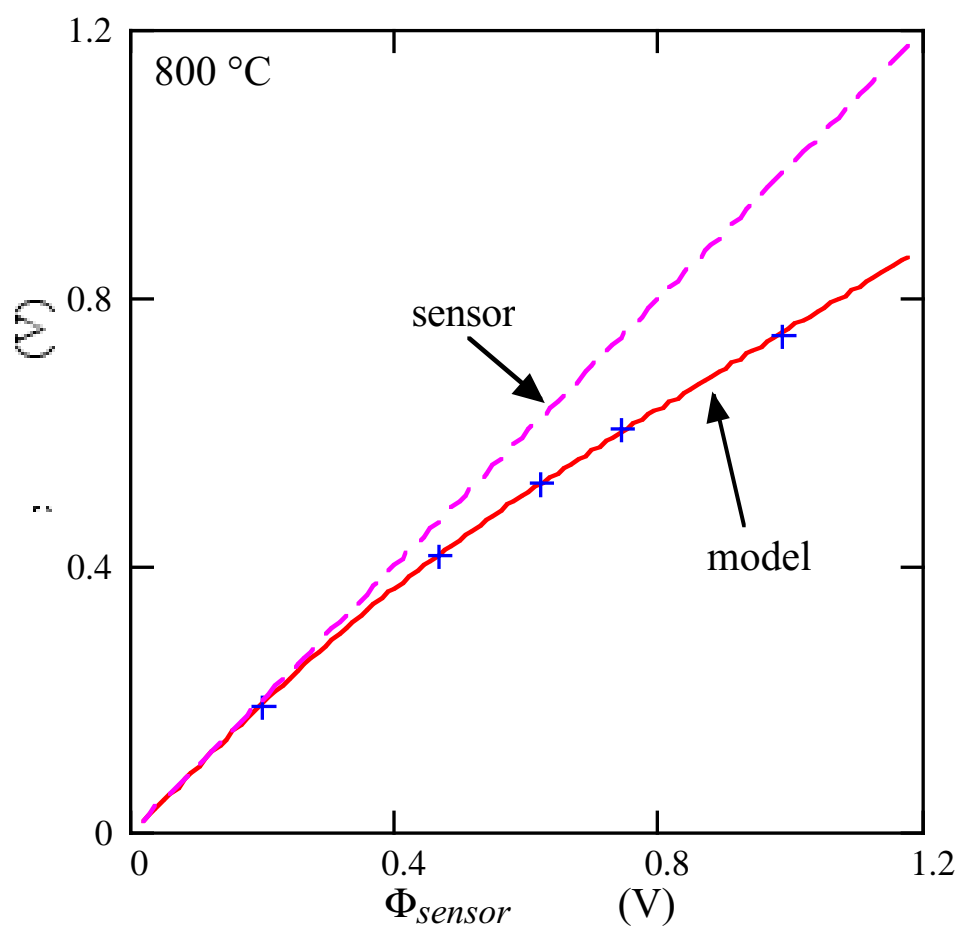

Figure 4-8. Open-circuit potential, $\Phi_{o c}$, versus $\Phi_{\text {sensor }}\left(\Phi_{t h}\right)$ at $800{ }^{\circ} \mathrm{C}$, model (-) fitted to experimental data (+) for the fuel cell; sensor (---).

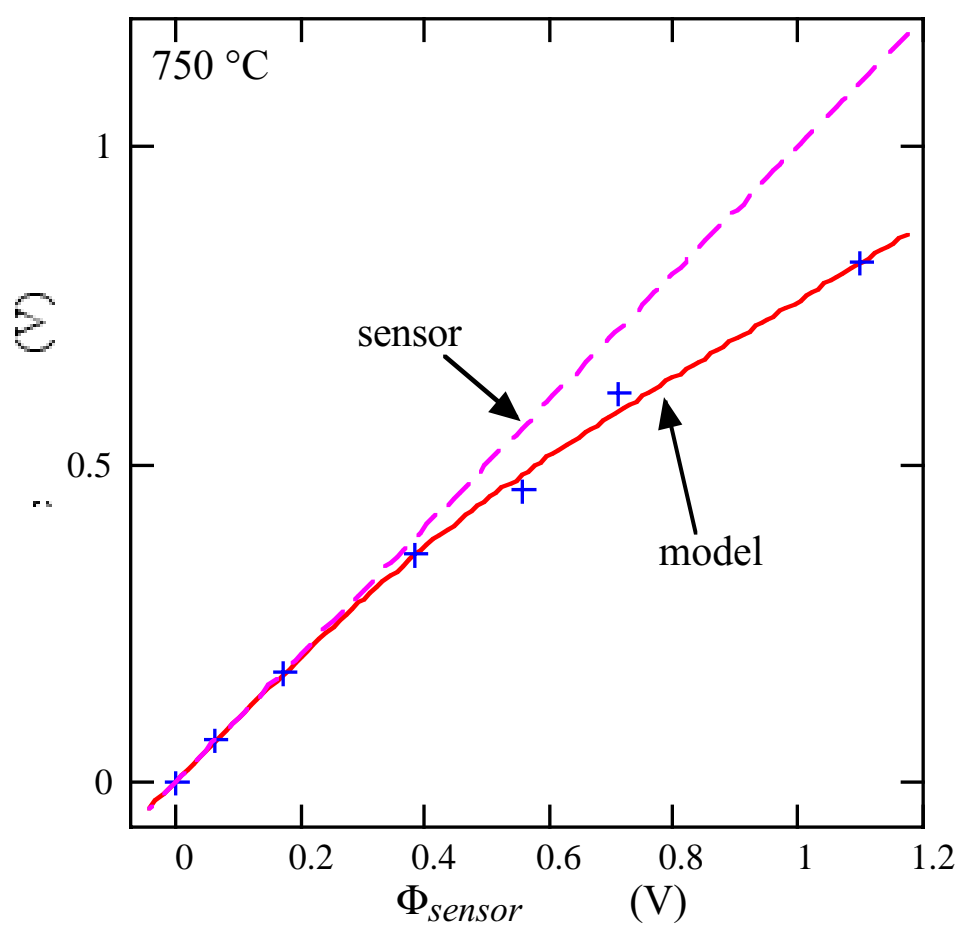

Figure 4-9. Open-circuit potential, $\Phi_{o c}$, versus $\Phi_{\text {sensor }}\left(\Phi_{t h}\right)$ at $750{ }^{\circ} \mathrm{C}$, model (-) fitted to experimental data (+) for the fuel cell; sensor (---). 


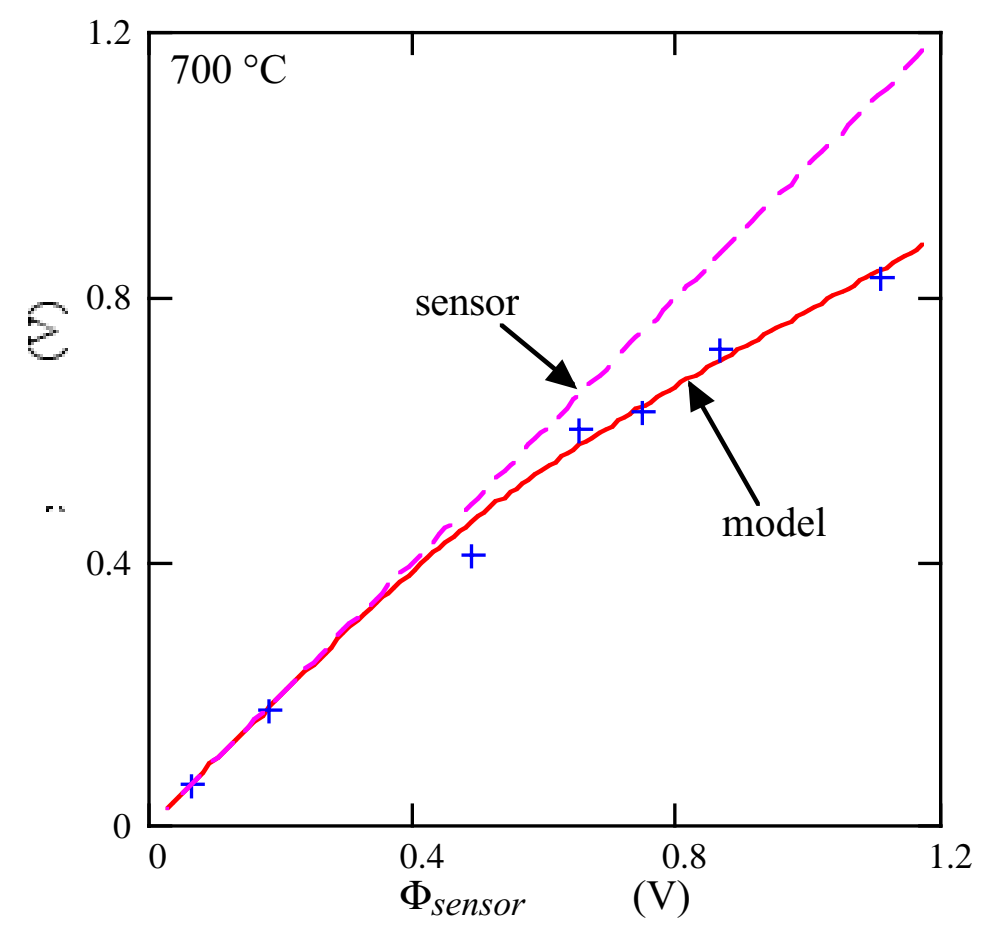

Figure 4-10. Open-circuit potential, $\Phi_{o c}$, versus $\Phi_{\text {sensor }}\left(\Phi_{t h}\right)$ at $700{ }^{\circ} \mathrm{C}$, model (一) fitted to experimental data (+) for the fuel cell; sensor (---).

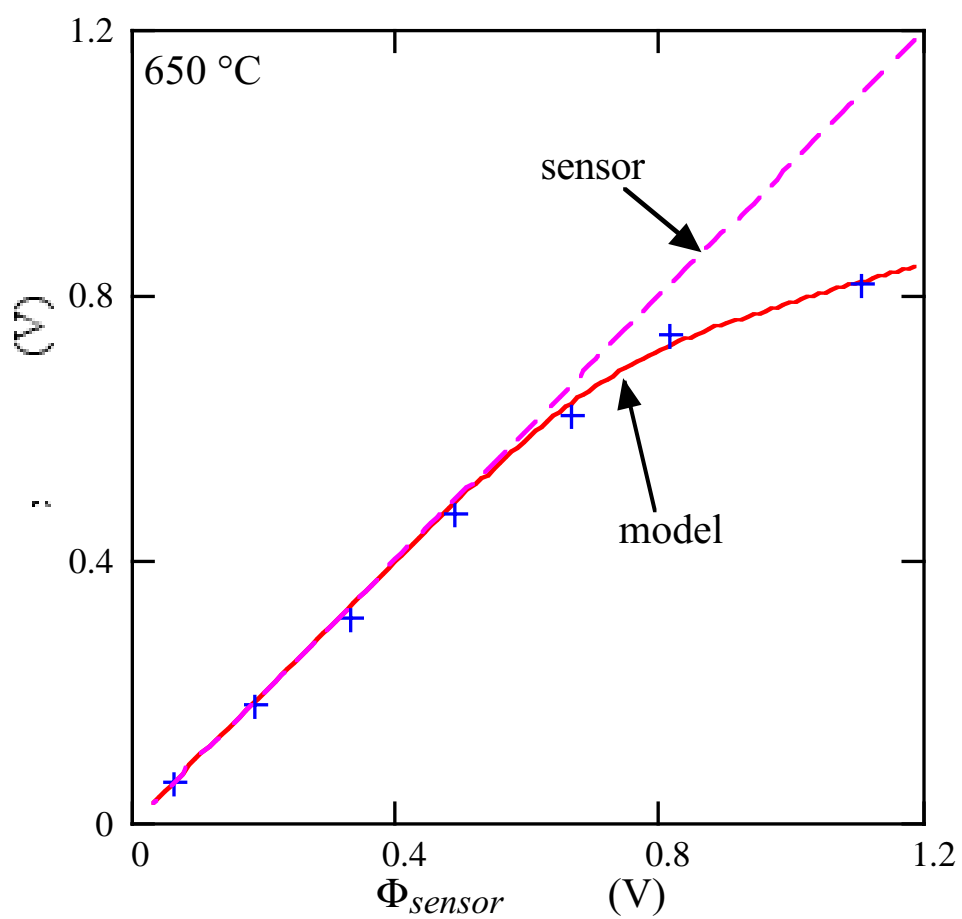

Figure 4-11. Open-circuit potential, $\Phi_{o c}$, versus $\Phi_{\text {sensor }}\left(\Phi_{t h}\right)$ at $650{ }^{\circ} \mathrm{C}$, model (一) fitted to experimental data (+) for the fuel cell; sensor (---). 


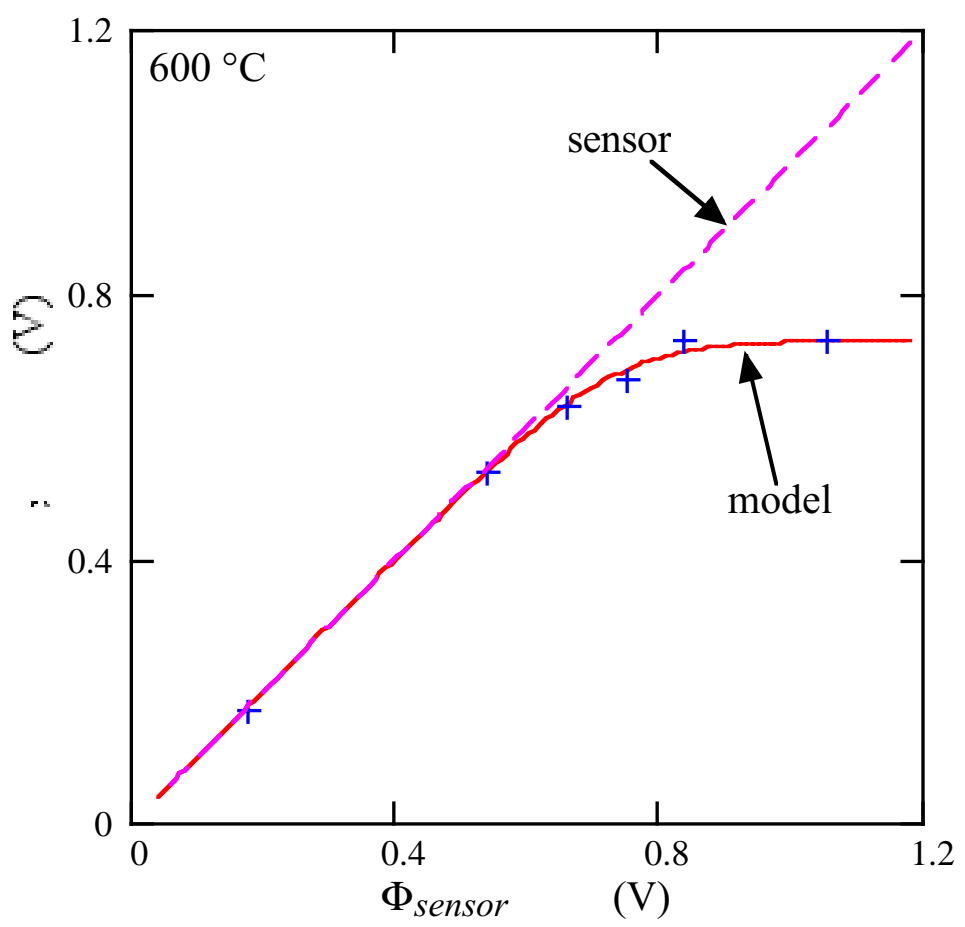

Figure 4-12. Open-circuit potential, $\Phi_{o c}$, versus $\Phi_{\text {sensor }}\left(\Phi_{t h}\right)$ at $600{ }^{\circ} \mathrm{C}$, model (-) fitted to experimental data (+) for the fuel cell; sensor (---).

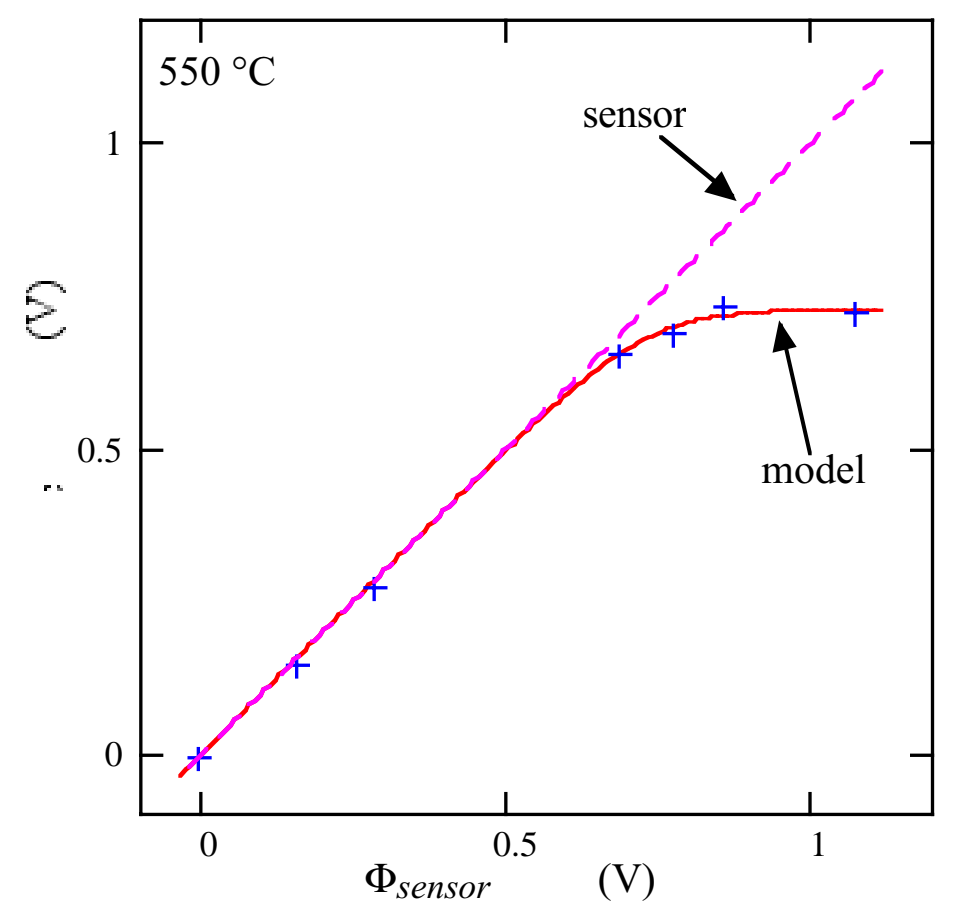

Figure 4-13. Open-circuit potential, $\Phi_{o c}$, versus $\Phi_{\text {sensor }}\left(\Phi_{t h}\right)$ at $550{ }^{\circ} \mathrm{C}$, model (-) fitted to experimental data $(+)$ for the fuel cell; sensor (---). 


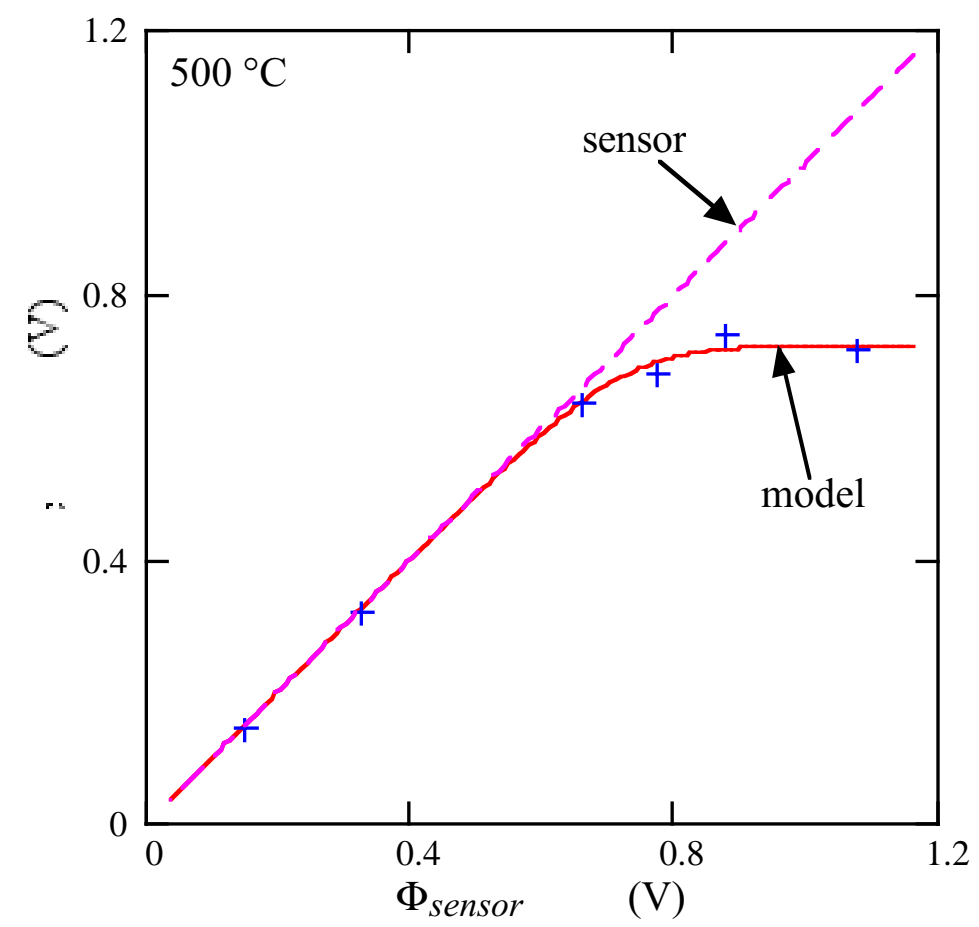

Figure 4-14. Open-circuit potential, $\Phi_{o c}$, versus $\Phi_{\text {sensor }}\left(\Phi_{t h}\right)$ at $500{ }^{\circ} \mathrm{C}$, model (一) fitted to experimental data (+) for the fuel cell; sensor (---).

As Figure 4-15 and Table 4-2. show, at $800{ }^{\circ} \mathrm{C}$ the values of $K_{r}$ and $D_{e} / D_{V}$ used to get the best fits to the data are respectively, larger and smaller than expected, i.e., $K_{r}$ and $D_{e} / D_{V}$ obtained at $800{ }^{\circ} \mathrm{C}$ do not concur with the values obtained in section 3.4. This outcome may be explained in two (complementary) ways. The first examines the impact of the (porous Pt) electrodes on the

Table 4-2. Values of $K_{r}$ and $D_{d} / D_{V}$ used to fit the model to experimental data

\begin{tabular}{cccccc}
\hline $\begin{array}{c}\text { Temperature } \\
\left({ }^{\circ} \mathrm{C}\right)\end{array}$ & $\begin{array}{c}K_{r} \\
\left(\mathrm{~m}^{-9} \mathrm{~atm} .{ }^{1 / 2}\right)\end{array}$ & $\ln K_{r}$ & $D_{e} / D_{V}$ & $r$ & $\breve{r}$ \\
\hline 800 & $1.0 \times 10^{72 \dagger}$ & 165.8 & $1.1 \times 10^{2 \dagger \dagger}$ & 0.958 & 0.929 \\
800 & $1.4 \times 10^{79}$ & 182.3 & 1.4 & 1.00 & 1.00 \\
750 & $1.6 \times 10^{78}$ & 180.1 & 1.6 & 0.999 & 0.999 \\
700 & $4.0 \times 10^{77}$ & 178.7 & 1.5 & 0.996 & 0.995 \\
650 & $5.6 \times 10^{73}$ & 169.8 & 5.6 & 0.999 & 0.998 \\
600 & $1.0 \times 10^{71}$ & 163.5 & 71 & 0.999 & 0.998 \\
550 & $4.5 \times 10^{66}$ & 153.5 & $5.7 \times 10^{3}$ & 0.999 & 0.999 \\
500 & $4.0 \times 10^{65}$ & 151.1 & $1.0 \times 10^{4}$ & 0.999 & 0.998 \\
\hline
\end{tabular}

$\dagger K_{r}$ calculated from thermogravimetric data of Kobayashi et al. [43] in section 3.4.1, Table 3-1.

$\dagger \dagger D_{e} / D_{V}$ calculated from Eq. (3.19) using data of Gödickemeier et al. [45, 46] in section 3.4.2, Table 3-2. 

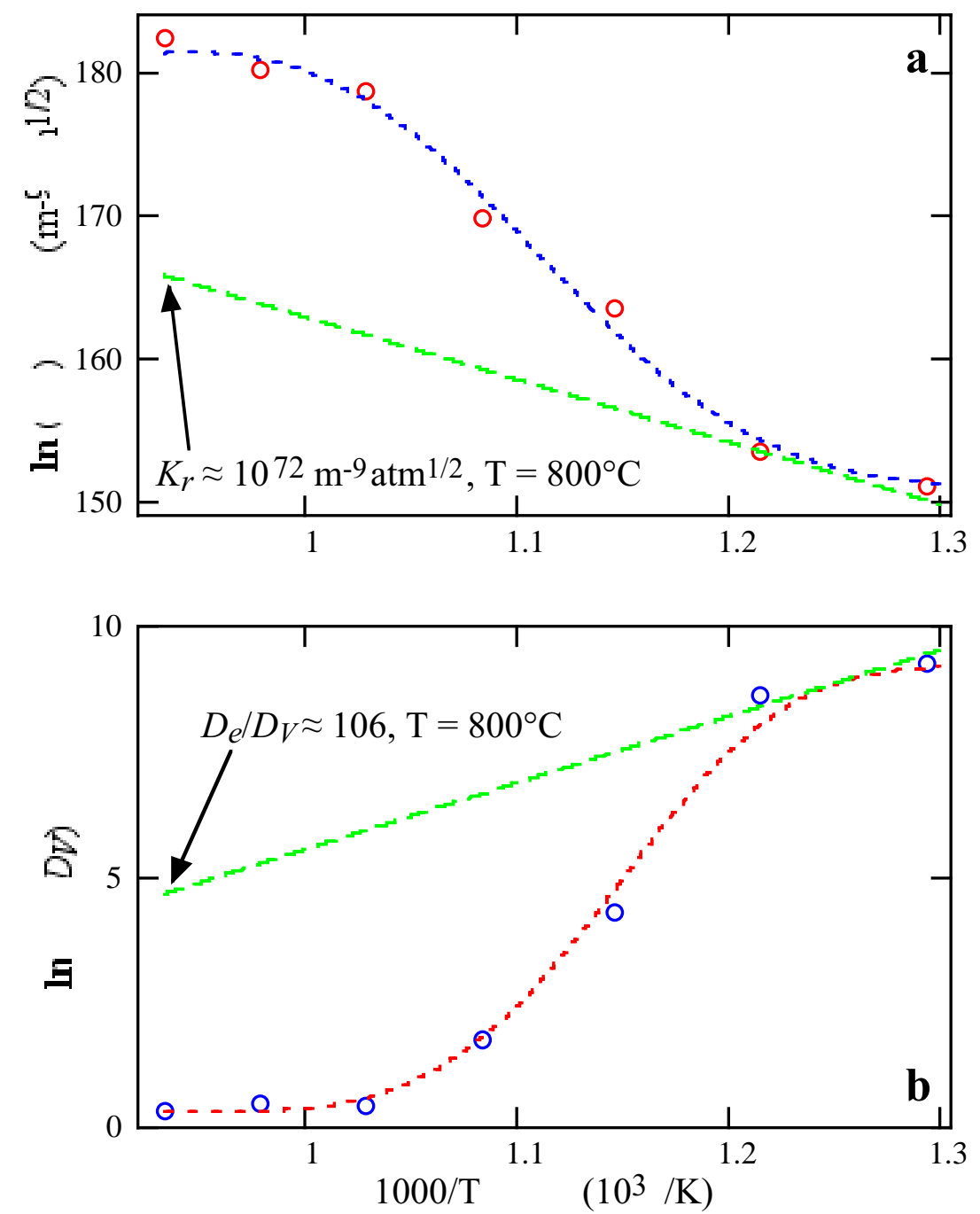

Figure 4-15. Plots of (a) $K_{r}$ and (b) $D_{e} / D_{V}$ versus reciprocal temperature. $K_{r}$ and $D_{e} / D_{V}$ values are those used in fitting the model (Figures 4-8 to 4-14). The dashed lines are for visual guidance and the straight lines indicate probable slopes without the impact of Pt electrodes, defect associates [36] and electrode overpotential.

characteristics of the SDC surface as they relate to $K_{r}$ and $D_{e} / D_{V}$. The second examines the sensitivity of $K_{r}$ and $D_{e} / D_{V}$ to experimental error.

Electrode Effects. Figure 4-16a shows surface sites where the oxygen exchange can occur distributed across the surface of the SDC sample in what may be described as their normal states (i.e., before application of the electrodes). When electrodes are introduced, (see Figure 416b) the nature of many of the surface sites changes. At the SDC-Pt-gas interface, (i.e., the triple-phase boundary) the oxygen exchange reaction is enhanced significantly because, for the cathodic reaction (i.e., oxygen incorporation), adsorbed oxygen species (from the gas phase) can 

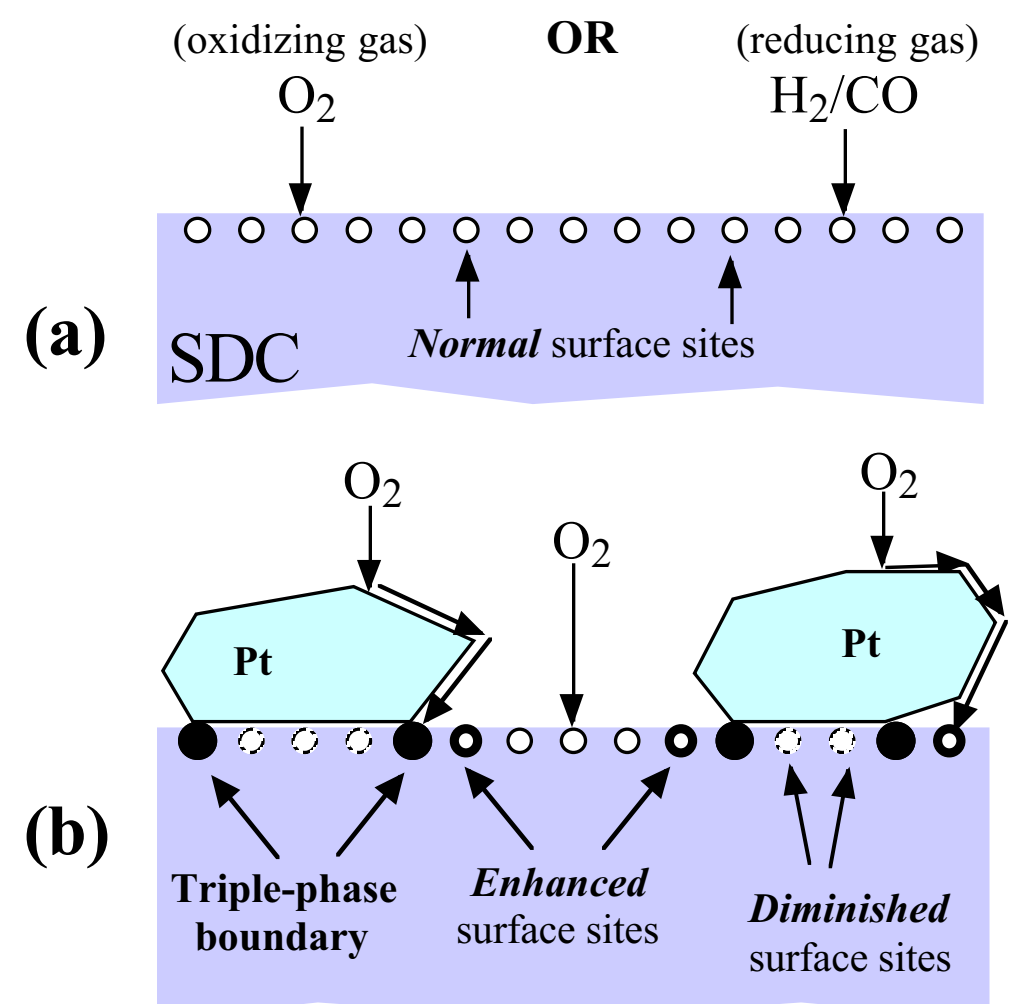

Figure 4-16. Comparison of the SDC-gas and SDC-Pt-gas interfaces showing: (a) Normal oxygen exchange surface sites for SDC in an oxidizing or a reducing gas, and (b) Enhanced and diminished oxygen exchange surface sites and triple phase (SDC-Pt-gas) boundaries.

obtain electron(s) readily (in comparison to SDC alone) from the metal phase. Adsorbed oxygen species are then transferred into a vacant oxygen site on the SDC (the ceramic phase) surface. This process is reversed for the anodic reaction (i.e., oxygen release) where oxygen atoms leave oxygen sites in the ceramic phase, release electrons to the metal phase and then react with the gas phase $^{8}$. The oxygen exchange reaction is further catalyzed by the platinum, which adsorbs oxygen (and, potentially, species from the reducing gas) more readily than SDC [40]. The adsorbed oxygen (cathode reaction) migrates to the triple-phase boundary or even to surface sites in its neighborhood that benefit from the spreading of electrons across the surface from the triple phase boundaries (i.e., the enhanced surface sites depicted in Figure 4-16b).

As temperature increases the (electrochemical) resistance of the electrodes (to ionic transport), and therefore the electrode overpotential, decreases. In addition, the electronic conductivity of the electrolyte increases with temperature, thereby reducing $\Phi_{\mathrm{oc}}$ and $t_{\text {ion }}^{\prime}$. Both

\footnotetext{
${ }^{8}$ This is a highly simplified description. The steps for the anodic reaction are very complex and involves several steps that have not yet been identified without ambiguity. A primary source of uncertainty for the anodic reaction is the presence (theoretically) of numerous paths for the reaction between fuel and oxygen species [40].
} 
have an effect on the performance of the SOFC, but the dominance of one over the other is dependent on the associated activation energies. Further discussion of these effects may be found in References 36, 40, 50 and 51.

As the temperature lowers, the significance of electrode overpotentials increases and becomes just as important as the change in $K_{r}$. In open-circuit $\left(I_{e x t}=0\right)$ conditions the dominant contributor to the electrode overpotential is slow electrocatalytic (charge transfer) reactions at the electrodes. At high enough temperatures, the electrocatalytic reactions are fast and the electrode overpotential can be neglected. This is not true at lower temperatures. As temperatures decrease, the charge transfer rate at the interface slows causing an accumulation of charged oxygen species near the reaction sites. The accumulation creates an electric field that slows down and eventually stops other charged oxygen species from approaching the reaction sites. Thermodynamically, the material should still be able to reach thermodynamic equilibrium (i.e., the charge transfer reactions will take place if given enough time). However, if the reaction kinetics are slow enough, thermodynamic equilibrium will not be attained. Consequently, the effective oxygen potential gradient and, therefore, the open-circuit potential, across the SDC sample is less than that predicted by thermodynamics.

Where possible, electrodes are designed to minimize the overpotential. The Pt electrodes on the sensor are specially designed to optimize the performance of the sensor by minimizing the electrode overpotential. In contrast, our simple porous $\mathrm{Pt}$ electrodes have not yet been optimized. Hence they produce a comparably larger overpotential, in similar conditions, than the electrodes on the commercial oxygen sensor.

Experimental Errors. The values of $K_{r}$ in Table 4-2 show that the difference between $K_{r}$ from the data of Kobayashi et al. [43] and that calculated from the model, at $800{ }^{\circ} \mathrm{C}$ is large. However, they also show that the corresponding values for $\ln K_{r}\left(\propto \Delta G_{r}\right)$ are within $10 \%$ of each other. The reason that this is significant is that the actual measured quantities in the experiment are $\Phi_{o c}$ and temperature and $\Phi_{o c}$ is proportional to $\ln K_{r}$ as shown below:

$$
\ln \frac{K_{r}}{K_{r}^{*}}=-\frac{\Delta G_{r}}{k T}=-\frac{2 z_{V} q \Phi_{o c}}{k T}
$$

where $K_{r}^{*}$ is a unit bearing pre-exponential (made necessary by the definition of $K_{r}$ in Eq. (3.14). Consequently, experimental errors made in the measurement of $\Phi_{o c}$ are magnified (exponentially) in the calculation of $K_{r}$. Moreover, from error analysis 


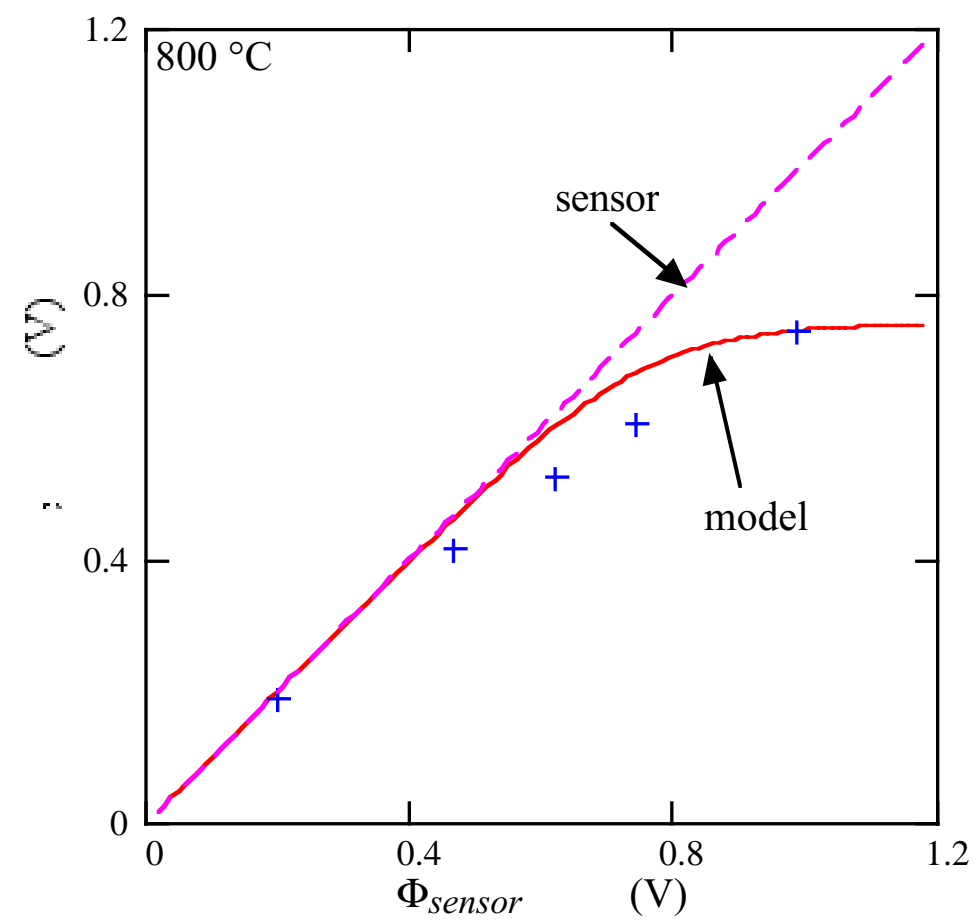

Figure 4-17. Open-circuit potential, $\Phi_{o c}$, versus $\Phi_{\text {sensor }}\left(\Phi_{t h}\right)$ at $800{ }^{\circ} \mathrm{C}$ without "fitting". The model (-) was fitted to experimental data (+) (sensor (---)) for the fuel cell using the $K_{r}$ value obtained from the data of Kobayashi et al. [43] and the corresponding $D_{e} / D_{V}$ ratio in Table 3-2.

$$
\frac{\Delta_{e r r}\left(\ln K_{r}-\ln K_{r}^{*}\right)}{\ln K_{r}-\ln K_{r}^{*}}=\frac{\Delta_{e r r} \Phi_{o c}}{\Phi_{o c}}+\frac{\Delta_{e r r} T}{T}
$$

where the symbol $\Delta_{e r r}$ indicates the error (so $\Delta_{e r r} y$ is the error in $y$ ).

For the experiment, $\Delta_{e r r} \Phi_{o c} / \Phi_{o c}$ (i.e., the fractional error) is between $\sim 0.05$ and $\sim 0.1$ while $\Delta_{e r r} T / T$ lies between $\sim 0.01$ and $\sim 0.05$. Therefore, the fractional error in $\ln K_{r} / K_{r}^{*}$ falls in the range 0.06 to 0.15 (i.e., $6 \%$ to $15 \%$ ). This result shows that, if experimental errors are included, the $K_{r}$ (or perhaps more appropriately $\ln K_{r}$ ) value obtained from Kobayashi et al. [43] is approximately equal to that obtained from the $\Phi_{o c}$ experiments outlined above. This argument is further corroborated by the good fit, Figure 4-17, obtained when the model is applied to experimental data using the $K_{r}$ value from Kobayashi et al. [43] and the corresponding $\Theta$ from Table 3-2.

Finally, there is also the effect of the $P_{\mathrm{O}_{2}}$ range in which the experiments were conducted. The data of Kobayashi et al. [43] (at $800{ }^{\circ} \mathrm{C}$ ) spans a $P_{\mathrm{O}_{2}}$ range from $\sim 10^{-17.55} \mathrm{~atm}$. to $\sim 10^{-20.5} \mathrm{~atm}$. while for the same temperature the $\Phi_{o c}$ experiment spans a range of $\sim 10^{-4}$ to $\sim 10^{-19}$ atm. In 
addition, the data of Kobayashi et al. [43], in Table 4, shows an increasing trend for $K_{r}$ as $P_{\mathrm{O}_{2}}$ decreases. Together, the implication is that had Kobayashi et al. [43] taken data over a larger range then, perhaps, a higher average $K_{r}$ value would have been obtained from their data. This result is substantiated somewhat when the model is applied to the $\Phi_{o c}$ data using the $K_{r}$ (and the corresponding $D_{e} / D_{V}$ ratio) value calculated from the data of Kobayashi et al. [43] shown in Figure 4-17. The data point, corresponding to $P_{0} \approx 10^{-19}$ atm., falls exactly on the modeled curve.

\section{BILAYER MODEL}

In this section, the bilayer model presented earlier is thoroughly revised and extended to closed-circuit conditions. Models that we developed elsewhere [36, 52] are used to obtain the relationship between the thickness ratio of the constituent layers of the SDC\ESB bilayer and (in open-circuit conditions) the chemical potential drop or (in closed-circuit conditions) the electrical potential drop across either layer. By having such a relationship, the relative thickness of the SDC and ESB layers may be optimized to maximize total conductivity of the bilayer and the protection of ESB from decomposition under a range of operating conditions.

\subsection{OPEN-CIRCUIT CONDITIONS AND THICKNESS RATIO}

Mechanistically, the diffusion of oxygen occurs by oxygen ions jumping from an occupied site to an adjacent vacant site in the MIECs oxygen sub-lattice. Also, oxygen vacancies and electrons/holes travel together in an electrically neutral packet (see Figure 3-1), as discussed earlier in section 3.2. Thus, the fluxes of the various species are related as follows:

$$
2 z_{V} j_{\mathrm{O}_{2}}=-z_{V} j_{V}=z_{e, h} j_{e, h}
$$

where $z_{e, h}$ is the charge equivalence of electrons (-1) or holes (+1).

For the bilayered electrolyte, the flux of any species is the same in both layers, in steady state conditions. Also, Eq. (5.1) shows that it is necessary to consider the flux of only one species. Therefore, for a bilayer consisting of an $\varepsilon$ layer (SDC) and a $\delta$ layer (ESB), we have:

$$
\begin{gathered}
j_{\mathrm{O}_{2}}^{\varepsilon}=j_{\mathrm{O}_{2}}^{\delta} \\
j_{V}^{\varepsilon}=j_{V}^{\delta}
\end{gathered}
$$




$$
z_{e, h} j_{e, h}^{\varepsilon}=z_{e, h} j_{e, h}^{\delta}
$$

where the superscript denotes the layer. At this point two approaches are taken. We have obtained [36] expressions for $j_{V}$ based on Wagner's equation [53] (the "thermodynamic" model) and the transport equations listed in section 3.1 (the transport model). Both approaches are used and the results compared below. However, we expect that the expression for $j_{V}$ derived from the transport equations [36] to be more accurate since fewer assumptions were made in its development. Nevertheless, the accuracy of both models (transport and thermodynamic) may, indirectly, be validated by comparing the results of both approaches.

\subsection{1 "Thermodynamic" Approach}

The oxygen vacancy and electron conductivity of the $n$-type MIEC, SDC, as a function of $P_{\mathrm{O}_{2}}$, is given by ${ }^{9}[36]$ :

$$
\sigma_{V}(P)=z_{V}^{2} q^{2} \frac{D_{V}}{k_{B} T}\left[\frac{3}{4} K_{r}^{\frac{1}{2}} P^{-\frac{1}{4}}+\left(\frac{1}{2} c_{A}\right)^{\frac{3}{2}}\right]^{\frac{2}{3}}
$$

and

$$
\sigma_{e}(P)=z_{e}^{2} q^{2} \frac{D_{e}}{k_{B} T} K_{r}^{\frac{1}{2}} P^{-\frac{1}{4}}\left[\frac{3}{4} K_{r}^{\frac{1}{2}} P^{-\frac{1}{4}}+\left(\frac{1}{2} c_{A}\right)^{\frac{3}{2}}\right]^{-\frac{1}{3}}
$$

Similarly, the oxygen vacancy and electron-hole conductivity of the $p$-type MIEC, ESB, as a function of $P_{\mathrm{O}_{2}}$, is given by ${ }^{10}[36,38]$ :

$$
\sigma_{V}(P)=\frac{z_{V}^{2} q^{2} D_{V}}{16 k_{B} T}\left[\left(K_{m} P^{\frac{1}{2}}+8 c_{A}\right)^{\frac{1}{2}}-K_{m}^{\frac{1}{2}} P^{\frac{1}{4}}\right]^{2}
$$

and

\footnotetext{
${ }^{9}$ For $P_{\mathrm{O}_{2}}<\sim 1$ atm. the electronic conduction in SDC (and GDC) is $n$-type [36, 47].

10 For $P_{\mathrm{O}_{2}}$ values above its decomposition potential, the electronic conduction in ESB is $p$-type [36, $\left.38-41\right]$.
} 


$$
\sigma_{h}(P)=\frac{z_{h}^{2} q^{2} D_{h}}{4 k_{B} T}\left(-K_{m} P^{\frac{1}{2}}+\sqrt{8 c_{A} K_{m} P^{\frac{1}{2}}+K_{m}^{2} P}\right)
$$

where $K_{m}$ is the external equilibrium constant $[36,38]$ for ESB and the subscript " $h$ " refers to electron-holes. It should also be noted that in applying these formulae to ESB the erbium and bismuth cations are treated as aliovalent acceptor "dopants" sitting in a fluorite lattice [54].

Wagner's equation [53] for oxygen flux may be combined with Eq. (5.1) with the result:

$$
-j_{O_{2}}=\frac{k_{B} T}{4^{2} q^{2} L^{\varepsilon}} \cdot \int_{P_{0}}^{P_{I}} \frac{\sigma_{V}^{\varepsilon}(P) \sigma_{e}^{\varepsilon}(P)}{\sigma_{V}^{\varepsilon}(P)+\sigma_{e}^{\varepsilon}(P)} \cdot \frac{d P}{P}=\frac{k_{B} T}{4^{2} q^{2} L^{\delta}} \cdot \int_{P_{I}}^{P_{L}} \frac{\sigma_{V}^{\delta}(P) \sigma_{h}^{\delta}(P)}{\sigma_{V}^{\delta}(P)+\sigma_{h}^{\delta}(P)} \cdot \frac{d P}{P}
$$

which may be rewritten as

$$
\tau\left(P_{I}\right)=\frac{L^{\varepsilon} j_{\mathrm{O}_{2}}^{\varepsilon}}{L^{\delta} j_{\mathrm{O}_{2}}^{\delta}}=\frac{L^{\varepsilon}}{L^{\delta}}=\frac{\int_{P_{0}}^{P_{I}} \frac{\sigma_{V}^{\varepsilon}(P) \sigma_{e}^{\varepsilon}(P)}{\sigma_{V}^{\varepsilon}(P)+\sigma_{e}^{\varepsilon}(P)} \cdot \frac{d P}{P}}{\int_{P_{I}}^{P_{L}} \frac{\sigma_{V}^{\delta}(P) \sigma_{h}^{\delta}(P)}{\sigma_{V}^{\delta}(P)+\sigma_{h}^{\delta}(P)} \cdot \frac{d P}{P}}
$$

where $\tau$ is defined as the SDCLESB $(\delta \backslash \varepsilon)$ thickness ratio of the two layers, L is the thickness (i.e., length) of a layer and the subscripts " 0 " and " $\mathrm{L}$ " refer to the conditions at $x=0$ (low $P_{\mathrm{O}_{2}}$ or fuel/reducing side of the SOFC/electrolyte) and $x=\mathrm{L}$ (high $P_{\mathrm{O}_{2}}$ or air/oxidizing side of the SOFC/electrolyte); the subscript " $I$ " refers to the interface between the two constituent layers.

The integral in the denominator in Eq. (5.7) exists, but it is very unwieldy. For ESB, $K_{m}$ $>10^{19} \mathrm{~m}^{-3} \mathrm{~atm} .{ }^{-1 / 2}$ and $c_{A}=2.2 \times 10^{28} \mathrm{~m}^{-3}$ [36], consequently, in the range of $P_{\mathrm{O}_{2}}\left(10^{-20} \mathrm{~atm}\right.$. to 0.21 atm. ) and $T\left(500{ }^{\circ} \mathrm{C}\right.$ to $\left.800{ }^{\circ} \mathrm{C}\right)$ under investigation

$$
8 c_{A} \gg K_{m} P^{1 / 2}
$$

and Eqs. (5.5) and (5.6) reduce to

$$
\sigma_{V}(P)=\frac{z_{V}^{2} q^{2} D_{V} c_{A}}{2 k_{B} T}
$$

and 


$$
\sigma_{h}(P)=\frac{z_{h}^{2} q^{2} D_{h}}{k_{B} T} K_{m}^{\frac{1}{2}} P^{\frac{1}{4}}\left(\frac{1}{2} c_{A}\right)^{\frac{1}{2}}
$$

With this simplification the denominator in Eq. (5.7) is easily integrated and Eq. (5.7) becomes

$$
\tau\left(P_{I}\right)=\frac{\frac{z_{e}^{2} D_{e}^{\varepsilon}}{1+\frac{4}{3} \Theta^{\varepsilon}}\left\{\frac{c_{V}^{\varepsilon}(P)}{2}-\frac{\Omega^{2}}{6} \ln \frac{c_{V}^{\varepsilon}(P)-\Omega \sqrt{c_{V}^{\varepsilon}(P)}+\Omega^{2}}{\left(\sqrt{c_{V}^{\varepsilon}(P)}+\Omega\right)^{2}}-\left.\frac{\Omega^{2}}{\sqrt{3}} \tan ^{-1} \frac{2 \sqrt{c_{V}^{\varepsilon}(P)}-\Omega}{\Omega \sqrt{3}}\right|_{P_{0}} ^{P_{I}}\right\}}{-\frac{z_{h}^{2} D_{h}^{\delta} c_{A}^{\delta}}{8 \Theta^{\delta}} \cdot\left\{\left.\ln \left(1+\Theta^{\delta}\left(\frac{1}{2} c_{A}^{\delta}\right)^{-\frac{1}{2}} K_{m}^{\frac{1}{2}} P^{\frac{1}{4}}\right)\right|_{P_{I}} ^{P_{L}}\right\}}
$$

where

$$
\begin{gathered}
\Omega=-\frac{4}{3} \Theta^{\frac{1}{3}}\left(\frac{1}{2} c_{A}^{\varepsilon}\right)^{\frac{1}{2}}\left(1+\frac{4}{3} \Theta\right)^{-\frac{1}{3}} \\
\Theta^{\varepsilon, \delta}=\frac{z_{e, h}^{2} D_{e, h}}{z_{V}^{2} D_{V}}
\end{gathered}
$$

and, from Eq. (3.15),

$$
c_{V}^{\varepsilon}(P)=\left[\frac{3}{4} K_{r}^{\frac{1}{2}} P^{-\frac{1}{4}}+\left(\frac{1}{2} c_{A}^{\varepsilon}\right)^{\frac{3}{2}}\right]^{\frac{2}{3}}
$$

\subsection{2 "Transport" Approach}

From our earlier work [36], $j_{V}$, as derived from the transport model, is given by:

$$
j_{V}=\frac{D_{V} D_{e, h}}{L}\left[\frac{\left(z_{V}-z_{e, h}\right)\left(c_{V_{0}}-c_{V_{L}}\right)}{z_{V} D_{V}-z_{e, h} D_{e, h}}+\frac{z_{e, h}\left(D_{e, h}-D_{V}\right) c_{A}}{\left(z_{V} D_{V}-z_{e, h} D_{e, h}\right)^{2}} \ln \frac{z_{V}^{2} D_{V} c_{V_{L}}+D_{e, h} c_{e, h_{L}}}{z_{V}^{2} D_{V} c_{V_{0}}+D_{e, h} c_{e, h_{0}}}\right]
$$

Combining this equation with Eq. (5.1) and rearranging yields 


$$
\tau\left(P_{I}\right)=\frac{D_{V}^{\varepsilon} D_{e}^{\varepsilon}\left[\frac{\left(z_{V}-z_{e}\right)\left(c_{V_{0}}^{\varepsilon}-c_{V_{I}}^{\varepsilon}\right)}{z_{V} D_{V}^{\varepsilon}-z_{e} D_{e}^{\varepsilon}}+\frac{z_{e}\left(D_{e}^{\varepsilon}-D_{V}^{\varepsilon}\right) c_{A}^{\varepsilon}}{\left(z_{V} D_{V}^{\varepsilon}-z_{e} D_{e}^{\varepsilon}\right)^{2}} \ln \frac{\left(z_{V} D_{V}^{\varepsilon}-z_{e} D_{e}^{\varepsilon}\right) z_{V} c_{V_{I}}^{\varepsilon}+z_{e} D_{e}^{\varepsilon} c_{A}^{\varepsilon}}{\left(z_{V} D_{V}^{\varepsilon}-z_{e} D_{e}^{\varepsilon}\right) z_{V} c_{V_{0}}^{\varepsilon}+z_{e} D_{e}^{\varepsilon} c_{A}^{\varepsilon}}\right]}{D_{V}^{\varepsilon} D_{h}^{\delta}\left[\frac{\left(z_{V}-z_{h}\right)\left(c_{V_{I}}^{\delta}-c_{V_{L}}^{\delta}\right)}{z_{V} D_{V}^{\varepsilon}-z_{h} D_{h}^{\delta}}+\frac{z_{h}\left(D_{h}-D_{V}^{\varepsilon}\right) c_{A}^{\delta}}{\left(z_{V} D_{V}^{\varepsilon}-z_{h} D_{h}^{\delta}\right)^{2}} \ln \frac{\left(z_{V} D_{V}^{\varepsilon}-z_{h} D_{h}^{\delta}\right) z_{V} c_{V_{L}}^{\delta}+z_{h} D_{h}^{\delta} c_{A}^{\delta}}{\left(z_{V} D_{V}^{\varepsilon}-z_{h} D_{h}^{\delta}\right) z_{V} c_{V_{I}}^{\delta}+z_{h} D_{h}^{\delta} c_{A}^{\delta}}\right]}
$$

where

$$
\begin{gathered}
c_{V_{k}}^{\varepsilon}\left(P_{k}\right)=\left[\frac{3}{4} K_{r}^{\frac{1}{2}} P_{k}^{-\frac{1}{4}}+\left(\frac{1}{2} c_{A}^{\varepsilon}\right)^{\frac{3}{2}}\right]^{\frac{2}{3}} \\
c_{V_{k}}^{\delta}\left(P_{k}\right)=\frac{1}{16}\left[\left(K_{m} P_{k}^{\frac{1}{2}}+8 c_{A}^{\delta}\right)^{\frac{1}{2}}-K_{m}^{\frac{1}{2}} P_{k}^{\frac{1}{4}}\right]^{2}
\end{gathered}
$$

and the subscript $k=0, I, L$.

\subsubsection{Results and Discussion}

The results of the model for open-circuit conditions are summarized in Table 5-1. Table 5-1 shows that there is very close agreement (enough, perhaps, to be indistinct experimentally) between the "thermodynamic" model-Eq. (5.7c)—and the "transport" model-Eq. (5.12) — giving credence to the propriety of both approaches.

In the table, the values for $K_{r}$ are calculated from the thermogravimetric data of Kobayashi et al. [43] and from fitting Eq. (3.19) to our experimental data (section 4.3). At 500 ${ }^{\circ} \mathrm{C}$ the value of $K_{r}$ is adjusted slightly to reflect $t_{i o n}^{\prime}=0.9$ for SDC, as obtained by other researchers [19]. We expect that value wasn't achieved in our experiments due to high electrode overpotentials from the Pt-paste electrodes. This was discussed in section 4.3.

Similarly, the values for $K_{m}$ are calculated from Eq. (3.19) using literature values [55 57] for $t_{i o n}^{\prime}\left(\Phi_{\text {oc }} / \Phi_{\mathrm{th}}\right)$ and assuming $2 D_{V}(\mathrm{SDC}) \approx D_{V}(\mathrm{ESB})[52]$ and $D_{e}(\mathrm{SDC}) \approx D_{h}(\mathrm{ESB})=D_{e, h}$ (therefore $\Theta^{\varepsilon}=0.5 \Theta^{\delta}$ ). In addition, $c_{A}^{\varepsilon}=5 \times 10^{27} \mathrm{~m}^{-3}, c_{A}^{\delta}=22 \times 10^{27} \mathrm{~m}^{-3}, P_{\mathrm{L}}=0.21 \mathrm{~atm}$. and $P_{0}=$ $10^{-20} \mathrm{~atm}$. in all calculations made in this section. Understandably, there exists some variability in the literature [19, 55 - 58] for the value $t_{i o n}^{\prime}$ and although this variability is small $(<3 \%)$ it does have a significant impact on the predictions of the model for $\tau$. 
Table 5-1. $\tau^{*}$ and ASR $(\mathrm{L}=10 \mu \mathrm{m})$ at $800{ }^{\circ} \mathrm{C}$ and $500{ }^{\circ} \mathrm{C}$ in open-circuit conditions.

\begin{tabular}{|c|c|c|c|c|c|c|c|c|}
\hline $\begin{array}{c}T \\
\left({ }^{\circ} \mathrm{C}\right) \\
\end{array}$ & $\begin{array}{c}K_{r} \\
\left(\mathrm{~m}^{-9} \mathrm{~atm}^{1 / 2}\right)\end{array}$ & $\begin{array}{c}K_{m} \\
\left(\mathrm{~m}^{-3} \mathrm{~atm} \cdot{ }^{-1 / 2}\right)\end{array}$ & $\Theta_{\mathrm{SDC}}\left(\Theta_{\mathrm{ESB}}\right)$ & $\begin{array}{c}t_{\text {ion }}^{\prime} \\
(\mathrm{SDC})\end{array}$ & $\begin{array}{c}t_{\text {ion }}^{\prime} \\
(\mathrm{ESB}) \\
\end{array}$ & $\tau_{\text {thermo }}^{*}$ & $\tau_{\text {transport }}^{*}$ & $\begin{array}{l}\mathrm{ASR}^{\dagger \dagger} \\
\left(\Omega \mathrm{cm}^{2}\right)\end{array}$ \\
\hline 800 & $1.0 \times 10^{72}$ & $2.0 \times 10^{23}$ & 部11.5(5.75) & 0.80 & 0.98 & 17.2 & 17.8 & 0.010 \\
\hline 800 & ${ }^{\dagger} 1.0 \times 10^{72}$ & $8.4 \times 10^{23}$ & 神11.5(5.75) & 0.80 & 0.96 & 8.5 & 8.8 & 0.010 \\
\hline 800 & ${ }^{\ddagger} 1.4 \times 10^{79}$ & $2.0 \times 10^{23}$ & $0.35(5.75)$ & 0.75 & 0.98 & 18000 & 24000 & 0.011 \\
\hline 800 & ${ }^{\ddagger} 1.4 \times 10^{79}$ & $8.4 \times 10^{23}$ & ${ }^{\ddagger} 0.35(5.75)$ & 0.75 & 0.96 & 9100 & 12000 & 0.011 \\
\hline 500 & $\$ 4.0 \times 10^{65}$ & $1.0 \times 10^{18}$ & ॠ2570(1285) & 0.84 & 0.99 & 9.8 & 9.8 & 0.12 \\
\hline 500 & $4.0 \times 10^{65}$ & $9.3 \times 10^{18}$ & $2570(1285)$ & 0.84 & 0.97 & 3.2 & 3.2 & 0.11 \\
\hline 500 & $5.1 \times 10^{64}$ & $1.0 \times 10^{18}$ & $2570(1285)$ & 0.90 & 0.99 & 4.6 & 4.6 & 0.12 \\
\hline 500 & $5.1 \times 10^{64}$ & $9.3 \times 10^{18}$ & $2570(1285)$ & 0.90 & 0.97 & 1.53 & 1.53 & 0.10 \\
\hline
\end{tabular}

$\dagger$ Calculated from thermogravimetric data of Kobayashi et al. [43] in section 3.4.1.

$\ddagger$ Obtained from fitting Eq. (3.19) to experimental data—see section 4.3 and Table 4-2.

$\dagger$ ASR, SDC: $0.011 \Omega \mathrm{cm}^{2}$ at $800{ }^{\circ} \mathrm{C} ; 0.13 \Omega \mathrm{cm}^{2}$ at $500{ }^{\circ} \mathrm{C}$; ESB: $0.0024 \Omega \mathrm{cm}^{2}$ at $800{ }^{\circ} \mathrm{C} ; 0.048 \Omega \mathrm{cm}^{2}$ at $500{ }^{\circ} \mathrm{C}$.

$\$$ Calculated from Eq. (3.19) to concur with literature values [55 - 57] for $t_{\text {ion }}^{\prime}$ (as discussed in text).

The area-specific resistance (ASR) of the bilayered electrolyte is included in Table 5-1 to evaluate how low of an ASR is possible in open-circuit conditions. The total ASR of the bilayer, (if the cross-sectional area is the same for both layers) is given by:

$$
A S R=\left(\frac{L^{\varepsilon}}{\sigma^{\varepsilon}}+\frac{L^{\delta}}{\sigma^{\delta}}\right)
$$

However, since $\tau=L^{\varepsilon} / L^{\delta}$ and the total thickness of the bilayer, $L=L^{\varepsilon}+L^{\delta}=(1+1 / \tau) L^{\varepsilon}$ then

$$
A S R=\frac{L}{1+\tau}\left(\frac{\tau}{\sigma^{\varepsilon}}+\frac{1}{\sigma^{\delta}}\right)
$$

For large $\tau$ (i.e., $\tau>\sim 2$ at $800{ }^{\circ} \mathrm{C}$ and $\tau>\sim 3$ at $500{ }^{\circ} \mathrm{C}$ ) Eq. (5.15) reduces to

$$
A S R \approx \frac{L}{\sigma^{\varepsilon}}
$$

-i.e., the ASR depends solely on the conductivity of SDC. For small $\tau$ (i.e., $\tau<\sim 0.02$ at $800{ }^{\circ} \mathrm{C}$ and $\tau<\sim 0.03$ at $500{ }^{\circ} \mathrm{C}$ ) Eq. (5.15) reduces to 


$$
A S R=\frac{L}{\sigma^{\delta}}
$$

-i.e., the ASR depends solely on the conductivity of ESB.

Using $K_{r}$ calculated from the data of Kobayashi et al. [43], the optimal (minimum) thickness ratio, $\tau^{*}$, to prevent decomposition of the ESB layer-ESB decomposes for $P_{I} \leq \sim 10^{-9}$ atm. [55 - 57] at $800{ }^{\circ} \mathrm{C}$-at the SDC-ESB interface, is $\sim 9$ or $\sim 18$ depending on whether $t_{\text {ion }}^{\prime}$ is 0.98 or 0.96 for ESB [55 - 57]. Samaria-doped ceria is more electronically conductive than ESB and so it permeates oxygen more easily. If the layers had the same thickness, the chemical potential drop would be greater across the more resistive layer (analogous to an electrical circuit). Consequently, to increase the chemical potential drop across the SDC layer and offer protection to the ESB layer requires a thickening of the SDC layer relative to the ESB layer. Accordingly, if the ESB layer is more permeable (i.e., smaller $t^{\prime}{ }_{i o n}$ ) then the chemical potential drop across it is smaller and a thinner layer of SDC (smaller $\tau^{*}$ ) can provide the same protection. By extension, this suggests that there is a trade-off between having a bilayer that produces near theoretical open-circuit potential and one that has the highest conductivity (due to the thinner SDC layer).

At $800{ }^{\circ} \mathrm{C}$ the $\mathrm{ASR}$ (for $\mathrm{L}=10 \mu \mathrm{m}$ ) of the bilayered electrolyte is approximately equal to that of a single-layer SDC electrolyte (i.e., the ESB layer has a negligible effect on the ASR of the bilayer). (At $800{ }^{\circ} \mathrm{C}$, the ASR is $0.011 \Omega \mathrm{cm}^{2}$ and $0.0024 \Omega \mathrm{cm}^{2}$ for SDC and ESB respectively.) This result is a consequence of the high $\tau^{*}$ values necessitated by the oxygen permeability of SDC and though this result is not ideal, it is still has promise because the ESB layer blocks conduction of electrons through the bilayer and, thereby should increase the efficiency of power delivery to an external load compared to SDC alone.

The results obtained using $K_{r}$ and $\Theta$ calculated from our experimental data for SDC at $800{ }^{\circ} \mathrm{C}$, section 4.3 , reflects the difficulties we experienced with the Pt-paste electrodes and emphasizes the need to separate electrode effects from the measured value of $\Phi_{\mathrm{oc}}$ in order to accurately determine the performance of the electrolyte. Although more reasonable, the $K_{r}$ and $\Theta$ values calculated from our experimental data at $500{ }^{\circ} \mathrm{C}$ likewise suffer from electrode effects, in this case evidenced in the lower than expected $t^{\prime}{ }_{i o n}$.

At $500{ }^{\circ} \mathrm{C}$, ESB decomposes at $P_{I} \leq \sim 10^{-16}$ atm. [55 - 57] and $\tau *$ ranges from $\sim 1.5$ to $\sim 10$ depending on whether $t^{\prime}$ ion 0.84 or 0.90 for SDC and 0.98 or 0.96 for ESB [55 - 57]. Again this value is approximately equal to that of a single-layer SDC electrolyte alone. (At $500{ }^{\circ} \mathrm{C}$, the ASR is $0.13 \Omega \mathrm{cm}^{2}$ and $0.048 \Omega \mathrm{cm}^{2}$ for SDC and ESB respectively.) 
As Table 5-1 shows, improvement in $\tau^{*}$ depends largely on whether $t^{\prime}$ ion for SDC increases more rapidly than $t_{i o n}^{\prime}$ for ESB. Or if $K_{r}$ (for SDC) reduces more rapidly with temperature than $K_{m}$ (for ESB). The equilibrium constants are related to the free energies, $\Delta G_{r}$ and $\Delta G_{m}$ respectively, of the oxygen exchange reaction and temperature as follows:

$$
\ln K_{r}=\ln K_{r}^{\circ}-\frac{\Delta G_{r}}{k_{B}} \cdot \frac{1}{T}
$$

and

$$
\ln K_{m}=\ln K_{m}^{\circ}-\frac{\Delta G_{m}}{k_{B}} \cdot \frac{1}{T}
$$

where $K_{r}^{\circ}$ and $K_{m}^{\circ}$ are unit bearing pre-exponentials. The expressions above show that the equilibrium constant for the external equilibrium reaction (between the atmosphere and the material) that occurs more readily (i.e., has a higher free energy) will vary more strongly with (reciprocal) temperature. SDC is known to react (i.e., incorporate or release oxygen) more readily with the ambient atmosphere than ESB, because of its ability to produce electrons. Consequently, it is reasonable to expect that as the temperature is lowered $K_{r}$ will decrease more rapidly than $K_{m}$-and hence $t_{i o n}^{\prime}$ will increase more rapidly with declining temperature for SDC compared to ESB. Therefore, it is reasonable to expect more favourable values of $\tau^{*}$ as the temperature decreases.

In either case, the variability of $\tau^{*}$ due to the values of $K_{r}^{\circ}, K_{m}^{\circ}$ and $\Theta$ (or $t^{\prime}{ }_{i o n}$ ) result in an insignificant change in the performance factor, i.e., the ASR, of the bilayer. Therefore basing $\tau^{*}$ on the most conservative estimates of $K_{r}^{\circ}, K_{m}^{\circ}$ and $\Theta$ (or $t^{\prime}{ }_{i o n}$ ) will result in a very low ASR of $\sim 0.01$ at $800{ }^{\circ} \mathrm{C}$ and an acceptably low ASR of $\sim 0.1$ at $500{ }^{\circ} \mathrm{C}$. Either ASR would enable a high power density SOFC if electrodes with suitable performance (i.e., low overpotential) were found.

\subsection{CLOSED-CIRCUIT CONDITIONS AND THICKNESS RATIO}

\subsubsection{Model}

For closed-circuit conditions, the principles and procedure are almost identical to those described and developed in the preceding sections for open-circuit conditions. However, there is one major difference. Specifically, in closed-circuit conditions, oxygen does not permeate through the MIEC by ambipolar diffusion, rather oxygen vacancies are transported at a rate 
proportional to the external current. Consequently, it may not be as appropriate to speak of a $P_{\mathrm{O}_{2}}$ at the interface (nor at any other point in either MIEC layer). Since there is no longer a $P_{\mathrm{O}_{2}}$ at the interface, a new gauge is required to indicate the stability of the ESB layer.

In open-circuit conditions there is an oxygen chemical potential difference across each layer (equivalent to a difference in $P_{\mathrm{O}_{2}}$ ). In closed-circuit conditions the oxygen chemical potential difference still exists between $x=0$ and $x=\mathrm{L}$, however inside any layer (i.e., for $0<x$ $<\mathrm{L}$ ) it is replaced by defect chemical potential differences and electrical potential differences. Additionally, the electric potential is related to the defect concentration (and therefore the defect chemical potential) [36]. Altogether, this suggests that in closed-circuit conditions, the electric potential difference across the ESB layer should be a suitable gauge of the stability of ESB at the SDC-ESB interface.

At $800{ }^{\circ} \mathrm{C}$, and with air on one side, an ESB electrolyte decomposes when the fuel side $P_{\mathrm{O}_{2}} \leq \sim 10^{-9}$ atm. [55 - 57]. This corresponds to a (Nernst) potential difference of $\sim 0.44 \mathrm{~V}$. Therefore, if the electrical potential difference across the ESB layer is held below $0.4 \mathrm{~V}$, then the ESB layer remains stable. Similarly, at $500{ }^{\circ} \mathrm{C}$, with air on one side, an ESB electrolyte, decomposes when the fuel side $P_{\mathrm{O}_{2}} \leq \sim 10^{-16}$ atm. [55 - 57] corresponding to a (Nernst) potential difference of $\sim 0.6 \mathrm{~V}$ (since $P_{\mathrm{L}}=0.21 \mathrm{~atm}$. on the oxidizing side). Therefore, the ESB layer should remain stable when the electrical potential difference across it is less than $0.6 \mathrm{~V}$.

To obtain an expression for $\tau(\Delta \phi)$ in closed-circuit conditions, consider the closed-circuit defect flux and the spatially varying Galvani potential, which are given by [36]:

$$
-\frac{j_{V}}{D_{V}}+-\frac{j_{e, h}}{D_{e, h}}=\frac{z_{V}-z_{e, h}}{z_{e, h}} \gamma=\frac{z_{V}-z_{e, h}}{z_{e, h}} \cdot \frac{\phi_{L}-\phi_{0}+\lambda\left(c_{V_{0}}-c_{V_{L}}\right)}{\lambda L}
$$

and

$$
\phi_{L}-\phi_{0}=\frac{z_{V} \frac{j_{V}}{D_{V}}+z_{e, h} \frac{j_{e, h}}{D_{e, h}}}{z_{V}\left(z_{V}-z_{e, h}\right) f \gamma} \ln \frac{c_{V_{L}}+\frac{j_{V}}{z_{V} D_{V} f \lambda \gamma}}{c_{V_{0}}+\frac{j_{V}}{z_{V} D_{V} f \lambda \gamma}}
$$

where, $\phi$ is potential, $f=q / k_{B} T$ and

$$
\lambda=\frac{z_{V}-z_{e, h}}{z_{e, h} c_{A} f}
$$


Since the ESB layer is a poor electronic conductor it blocks the flux of electrons and holes. Additionally, as one moves away from open-circuit conditions, the electronic flux inside the MIEC decreases. Hence, Eqs. (5.16) and (5.17) reduce to

$$
-\frac{j_{V}}{D_{V}} \approx \frac{z_{V}-z_{e, h}}{z_{e, h}} \gamma=\frac{z_{V}-z_{e, h}}{z_{e, h}} \cdot \frac{\phi_{L}-\phi_{0}+\lambda\left(c_{V_{0}}-c_{V_{L}}\right)}{\lambda L}
$$

and

$$
\phi_{L}-\phi_{0} \approx \frac{z_{V} j_{V}}{z_{V}\left(z_{V}-z_{e, h}\right) D_{V} f \gamma} \ln \frac{c_{V_{L}}+\frac{j_{V}}{z_{V} D_{V} f \lambda \gamma}}{c_{V_{0}}+\frac{j_{V}}{z_{V} D_{V} f \lambda \gamma}}=-\frac{1}{z_{e, h} f} \ln \frac{c_{V_{L}}-\frac{c_{A}}{z_{V}}}{c_{V_{0}}-\frac{c_{A}}{z_{V}}}
$$

Combining Eqs. (5.1), (5.16b) and (5.17b) yields

$$
\tau(\Delta \phi)=\frac{D_{V}^{\varepsilon} \frac{z_{V}-z_{e}}{z_{e}}\left\{\frac{\Delta \phi^{\varepsilon}}{\lambda^{\varepsilon}}+\left(c_{V_{0}}^{\varepsilon}-\frac{c_{A}^{\varepsilon}}{z_{V}}\right)\left[1-\exp \left(-z_{e} f \Delta \phi^{\varepsilon}\right)\right]\right\}}{D_{V}^{\delta} \frac{z_{V}-z_{h}}{z_{h}}\left\{\frac{\Delta \phi^{\delta}}{\lambda^{\delta}}-\left(c_{V_{L}}^{\delta}-\frac{c_{A}^{\delta}}{z_{V}}\right)\left[1-\exp \left(z_{h} f \Delta \phi^{\delta}\right)\right]\right\}}
$$

where

$$
\Delta \phi^{\varepsilon}=\phi_{I}-\phi_{0}
$$

and

$$
\Delta \phi^{\delta}=\phi_{L}-\phi_{I}
$$

Additionally, by definition [36]

$$
\Delta \Phi=-\Delta \phi=-\left(\phi_{L}-\phi_{0}\right)
$$

where $\Delta \Phi$ is the potential across a single layered electrolyte. Thus, for the bilayer one may write 


$$
\Delta \Phi=\Delta \Phi^{\varepsilon}+\Delta \Phi^{\delta}=-\left(\phi_{I}-\phi_{0}\right)-\left(\phi_{L}-\phi_{I}\right)=-\Delta \phi^{\varepsilon}-\Delta \phi^{\delta}
$$

Substituting Eq. (5.23) for $-\Delta \phi^{\varepsilon}$, into Eq. (5.19) yields

$$
\tau\left(\Delta \phi^{\delta}\right)=-\frac{D_{V}^{\varepsilon} \frac{z_{V}-z_{e}}{z_{e}}\left\{\frac{\Delta \Phi+\Delta \phi^{\delta}}{\lambda^{\varepsilon}}-\left(c_{V_{0}}^{\varepsilon}-\frac{c_{A}^{\varepsilon}}{z_{V}}\right)\left[1-\exp \left(z_{e} f\left(\Delta \Phi+\Delta \phi^{\delta}\right)\right)\right]\right\}}{D_{V}^{\delta} \frac{z_{V}-z_{h}}{z_{h}}\left\{\frac{\Delta \phi^{\delta}}{\lambda^{\delta}}-\left(c_{V_{L}}^{\delta}-\frac{c_{A}^{\delta}}{z_{V}}\right)\left[1-\exp \left(z_{h} f \Delta \phi^{\delta}\right)\right]\right\}}
$$

Finally, the external equilibrium constants $K_{r}$ and $K_{m}$-Eqs. (5.13) and (5.14)—are functions of $\Delta \Phi^{\varepsilon}$ and $\Delta \Phi^{\delta}$ respectively and are given by [36]:

$$
K_{r}(\Delta \Phi)=K_{r}^{*} \exp \left(-\frac{\Delta G_{r} \pm z_{e} q \Delta \Phi}{k T}\right)
$$

and

$$
K_{m}(\Delta \Phi)=K_{m}^{*} \exp \left(-\frac{\Delta G_{m} \pm z_{h} q \Delta \Phi}{k T}\right)
$$

\subsubsection{Results and Discussion}

The results of the model for closed-circuit conditions are summarized in Table 5-2, where $\tau^{*}$ is referenced to $\Delta \Phi=\Phi_{t h}$ (i.e., short-circuit conditions, [36] where the voltage drop across either layer is greatest). The optimal thickness ratio to prevent decomposition of the ESB layer, in closed-circuit conditions-ESB decomposes for $-\Delta \phi^{\delta} \geq \sim 0.4 \mathrm{~V}$ at $800{ }^{\circ} \mathrm{C}$ and $-\Delta \phi^{\delta} \geq \sim 0.6 \mathrm{~V}$ at $500{ }^{\circ} \mathrm{C}$-is a function of the operating conditions. If the internal electronic current (the leakage current, $\left.I_{e}\right)$ is small, then external current $\left(I_{e x t}\right)$ is directly proportional to the internal ionic current $\left(I_{V}\right)$. Neglecting the effect of the electrodes,

$$
\Delta \Phi=t_{i o n}^{\prime} \Phi_{t h}-I_{e x t} R_{l o a d}
$$

where $R_{\text {load }}$ is the resistance of the external circuit. In short-circuit conditions, see Figure 5-1, $R_{\text {load }}=0$ and $t_{\text {ion }}^{\prime} \approx 1$ [36] (because $I_{e} \approx 0$ ). (Under this condition, the internal ionic current is maximum and $\Phi_{t h}=I_{V} \times R_{\text {int }}$.) Therefore, the largest values of $\Delta \Phi$ and $-\Delta \phi$ occur in short-circuit 
Table 5-2. $\tau^{*}$ — with reference to $\Delta \Phi=\Phi_{t h}$ (short-circuit conditions) —and ASR $(\mathrm{L}=10 \mu \mathrm{m})$ at $800{ }^{\circ} \mathrm{C}$ and $500{ }^{\circ} \mathrm{C}$ in closed-circuit conditions.

\begin{tabular}{cccccc}
\hline$T\left({ }^{\circ} \mathrm{C}\right)$ & $K_{r}\left(\mathrm{~m}^{-9} \mathrm{~atm} .{ }^{1 / 2}\right)$ & $K_{m}\left(\mathrm{~m}^{-3} \mathrm{~atm} .^{-1 / 2}\right)$ & $\Theta_{\mathrm{SDC}}\left(\Theta_{\mathrm{ESB}}\right)$ & $\tau^{*}$ & $\mathrm{ASR}^{\dagger \dagger}\left(\Omega \mathrm{cm}^{2}\right)$ \\
\hline 800 & $\ddagger 1.0 \times 10^{72}$ & $2.0 \times 10^{23}$ & $\ddagger 11.5(5.75)$ & 0.42 & 0.005 \\
800 & $\ddagger 1.0 \times 10^{72}$ & $8.4 \times 10^{23}$ & $\ddagger 11.5(5.75)$ & 0.42 & 0.005 \\
500 & $\ddagger 4.0 \times 10^{65}$ & $1.0 \times 10^{18}$ & $\ddagger 2570(1285)$ & 0.027 & 0.05 \\
500 & $\ddagger 4.0 \times 10^{65}$ & $9.3 \times 10^{18}$ & $\ddagger 2570(1285)$ & 0.027 & 0.05 \\
500 & $5.1 \times 10^{64}$ & $1.0 \times 10^{18}$ & $\ddagger 2570(1285)$ & 0.027 & 0.05 \\
500 & $5.1 \times 10^{64}$ & $9.3 \times 10^{18}$ & $\ddagger 2570(1285)$ & 0.027 & 0.05 \\
\hline
\end{tabular}

$\dagger$ Calculated from thermogravimetric data of Kobayashi et al. [43] in section 3.4.1.

\$ Obtained from fitting Eq. (3.19) to experimental data—see section 4.3 and Table 4-2.

$\dagger \dagger$ ASR, SDC: $0.011 \Omega \mathrm{cm}^{2}$ at $800{ }^{\circ} \mathrm{C} ; 0.13 \Omega \mathrm{cm}^{2}$ at $500{ }^{\circ} \mathrm{C}$; ESB: $0.0024 \Omega \mathrm{cm}^{2}$ at $800{ }^{\circ} \mathrm{C} ; 0.048 \Omega \mathrm{cm}^{2}$ at $500{ }^{\circ} \mathrm{C}$.

$\$$ \$ Calculated from Eq. (3.19) to concur with literature values [55 - 57] for $t_{\text {ion }}^{\prime}$ (as discussed in text).

conditions because that is the point at which the external circuit draws the largest current from the fuel cell-and $I_{e}$ is the smallest. In this context, $\tau^{*}$ is then the optimal thickness ratio with respect to short-circuit conditions and at $800{ }^{\circ} \mathrm{C}, \tau^{*} \approx 0.42$ while at $500{ }^{\circ} \mathrm{C} \tau^{*} \approx 0.027$. This is in stark contrast to the results obtained above for open-circuit conditions, Table 5-1. The difference however is readily explained by considering that in open-circuit conditions ESB is more resistive than SDC to the transport (permeation) of oxygen because of the low concentration of electronic species in ESB.

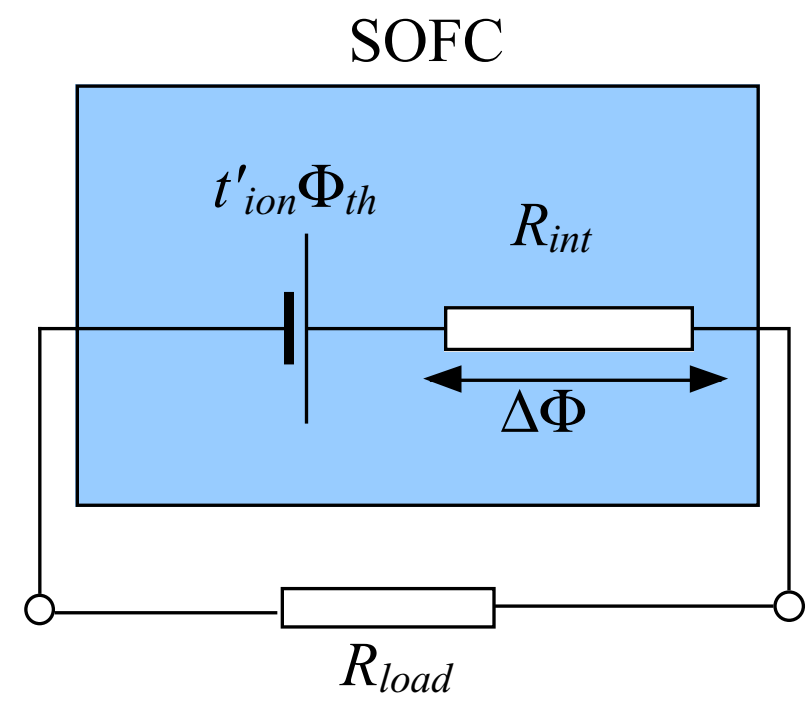

Figure 5-1. Equivalent circuit for SOFC 
In closed-circuit conditions (where the ionic and electronic species are no longer constrained to travel together) SDC is more resistive than ESB to the transport of oxygen vacancies because ESB has a higher concentration of oxygen vacancies than SDC. Hence, if the layers had the same thickness, there would be a larger electrical potential drop across the SDC layer. So to allow a larger voltage drop (up to $0.4 \mathrm{~V}$ at $800{ }^{\circ} \mathrm{C}$ or up to $0.6 \mathrm{~V}$ at $500{ }^{\circ} \mathrm{C}$ ) across the ESB layer, the thickness of the SDC layer must be reduced relative to that of the ESB layer.

Consequently, in closed-circuit conditions, the total ASR of the bilayer at $800{ }^{\circ} \mathrm{C}$ is $45 \%$ of the ASR of a single SDC layer of the same thickness-or approximately twice the ASR of a single ESB layer of the same thickness. At $500{ }^{\circ} \mathrm{C}$, even better results are obtained where the total ASR of the bilayer is $\sim 38 \%$ of the ASR of a single SDC layer of the same thickness or approximately equal to the ASR of a single ESB layer of the same thickness.

Table 5-2 also shows that $\tau^{*}$ (and therefore the ASR) is essentially independent of $t_{\text {ion }}^{\prime}$ (also $K_{r}$ and $K_{m}$ ). This is a consequence of the leakage current being negligibly small in shortcircuit conditions-when the electronic current travels through the external circuit and the electrolyte behaves like a perfect ionic conductor $\left(t_{i o n}^{\prime}=1\right)$-and because the ESB layer blocks electronic current.

Short-circuit conditions, provide us with a worst case scenario for a closed-circuit SOFC, i.e., an SOFC delivering power to a load. However, we are even more interested in the optimal delivery of that power. Again neglecting the effect of the electrodes,

$$
W=I_{\text {ext }} \Delta \Phi=I_{\text {ext }} t_{\text {ion }}^{\prime} \Phi_{\text {th }}-I_{\text {ext }}^{2} R_{\text {load }}
$$

where $W$ is the power delivered to the load. Differentiating Eq. (5.28) and setting the result to zero we are able to find the current at which maximum power, $W_{\max }$, is delivered. Then, by substituting that expression into Eq. (5.27), we have

$$
\Delta \Phi_{W_{\max }}=\frac{1}{2} t_{i o n}^{\prime} \Phi_{t h} \approx \frac{1}{2} \Phi_{t h}
$$

where, $\Delta \Phi_{\mathrm{W}_{\max }}$ is $\Delta \Phi$ for maximum power delivery.

Table 5-3 shows the results of the model for $\tau$ and ASR when the bilayer is optimized for maximum power delivery ( $\tau^{*}{ }_{w_{\max }}$ and $\mathrm{ASR}_{\mathrm{w}_{\max }}$ respectively). The values of $\Theta$ are omitted from Table 5-3 since they correspond exactly to those in Table 5-2. At $500{ }^{\circ} \mathrm{C}$ the decomposition potential for $\operatorname{ESB}(\sim 0.6 \mathrm{~V})$ is greater than $\Delta \Phi_{\mathrm{w}_{\max }}\left(\approx \Phi_{\mathrm{th}} / 2=0.37 \mathrm{~V}\right)$, therefore $\tau^{*}=0$ and the 
Table 5-3. $\tau^{*}{ }_{\mathrm{w}_{\max }}$ and ASR $(\mathrm{L}=10 \mu \mathrm{m})$ at $800^{\circ} \mathrm{C}$ and $500^{\circ} \mathrm{C}$ in closed-circuit conditions.

\begin{tabular}{ccccc}
\hline$T\left({ }^{\circ} \mathrm{C}\right)$ & $K_{r}\left(\mathrm{~m}^{-9} \mathrm{~atm} .^{1 / 2}\right)$ & $K_{m}\left(\mathrm{~m}^{-3} \mathrm{~atm} .^{-1 / 2}\right)$ & $\tau^{*} \mathrm{w}_{\max }$ & $\mathrm{ASR}_{\mathrm{W}_{\max } \dagger}\left(\Omega \mathrm{cm}^{2}\right)$ \\
\hline 800 & $\dagger 1.0 \times 10^{72}$ & $2.0 \times 10^{23}$ & 0.051 & 0.003 \\
800 & $\ddagger 1.0 \times 10^{72}$ & $8.4 \times 10^{23}$ & 0.051 & 0.003 \\
500 & n.a. & $1.0 \times 10^{18}$ & 0 & 0.048 \\
500 & n.a. & $9.3 \times 10^{18}$ & 0 & 0.048 \\
\hline
\end{tabular}

$\dagger$ Calculated from thermogravimetric data of Kobayashi et al. [43] in section 3.4.1.

\$ Obtained from fitting Eq. (3.19) to experimental data—see section 4.3 and Table 4-2.

$\dagger \dagger$ ASR, SDC: $0.011 \Omega \mathrm{cm}^{2}$ at $800{ }^{\circ} \mathrm{C} ; 0.13 \Omega \mathrm{cm}^{2}$ at $500{ }^{\circ} \mathrm{C}$; ESB: $0.0024 \Omega \mathrm{cm}^{2}$ at $800{ }^{\circ} \mathrm{C} ; 0.048 \Omega \mathrm{cm}^{2}$ at $500{ }^{\circ} \mathrm{C}$.

ASR is the same as that of an ESB layer. Table 5-3 shows that $\tau{ }^{*}{ }_{\mathrm{w}_{\max }}$ and $\mathrm{ASR}_{\mathrm{w}_{\max }}$ are significantly smaller than $\tau^{*}$ and ASR in Table 5-2, a direct consequence of the reduced operating voltage needed for maximum power delivery versus maximum current delivery (i.e., short-circuit conditions). For maximum power delivery, the ASR of the bilayer is just $\sim 25 \%$ greater than the ASR of a single ESB layer of the same thickness.

\subsection{SUMMARY}

The feasibility of a bilayered electrolyte consisting of a layer of SDC on the fuel (reducing side) side and ESB on the air (oxidizing) side of an SOFC has been studied. Expressly, the feasibility of the bilayered electrolyte depends on the optimization of the thickness ratio of the layers so that the ESB layer is protected from decomposition in a highly reducing (low $P_{\mathrm{O}_{2}}$ ) atmosphere while the ionic conductance of the combination is maximized. Consequently, it is necessary to obtain a stability criterion for ESB and its functional relationship to $\tau$. It was argued that in open-circuit conditions it is sufficient to know the $P_{\mathrm{O}_{2}}$ at the SDC-ESB interface while in closed-circuit conditions it is necessary to know the voltage drop, $-\Delta \phi^{\delta}$, across the ESB layer.

In open-circuit conditions, ("thermodynamic" and "transport") models developed for oxygen flux were used to obtain the functional relationship between the $\tau$ and the effective $P_{\mathrm{O}_{2}}$ at the SDC-ESB interface. Both approaches produced essentially identical results, which further substantiates their validity.

Using our best estimates of $K_{r}$ and $K_{m}$ at $800{ }^{\circ} \mathrm{C}$, the bilayer model showed that $\tau^{*}$ lies between 9 and 18 in open-circuit conditions. In closed-circuit conditions $\tau *$ is $\sim 0.4$ if the bilayer is optimized for maximum current delivery and $\sim 0.05$ for maximum power delivery. Similarly, 
at $500{ }^{\circ} \mathrm{C}$, $\tau$ lies between 1.5 and 10 in open-circuit conditions and is $\sim 0.03$ in closed-circuit conditions and $\tau^{*} \rightarrow 0$ at maximum power delivery

Since ESB has a higher ionic conductivity than SDC, a lower ASR for the bilayer is obtained for the smaller value of $\tau$ and, for the most efficient delivery of power, one would prefer the lowest ASR. This implies that an SDC-ESB bilayered SOFC would have the best performance if it was fabricated with a $\tau^{*}$ based on closed-circuit conditions-for maximum delivery of either power or current. This is not a problem since, in principle, a fuel cell is used to deliver current/power to a load. Moreover, open-circuit conditions in the presence of a reducing gas can be avoided by purging the gas or to just allow the fuel cell to cool since the decomposition $P_{\mathrm{O}_{2}}$ for ESB decreases with temperature. The latter solution is attractive because of its simplicity, however it would have to be carefully implemented since the effective $P_{\mathrm{O}_{2}}$ of the fuel also reduces with temperature.

\section{BILAYER: EXPERIMENTAL RESULTS}

In this section, initial results of experiments on SOFCs with SDCIESB bilayered electrolytes are presented.

\subsection{OPEN CIRCUIT VOLTAGE}

The open-circuit voltage of an oxygen concentration cell of the type:

$$
P_{\mathrm{O}_{2}}, \mathrm{Au} / \mathrm{ESB} \backslash \mathrm{SDC} / \mathrm{Pt}, P_{\mathrm{CO} / \mathrm{CO}_{2}}\left(\text { or } P_{\mathrm{H}_{2} / \mathrm{H}_{2} \mathrm{O}}\right)
$$

was measured as a function of $P_{\mathrm{O}_{2}}$ on the anode (fuel) side and temperature and used to calculate $t_{i o n}^{\prime}$. Au paste was coated on the ESB layer for a cathode and Pt paste was coated on the SDC layer for an anode. Figures 6-1 and 6-2 compare $\Phi_{o c}$ and $t_{i o n}$, respectively, obtained for single layer SDC (Eq. (4.1)) and bilayered SDC\ESB (Eq. (6.1)) cells. The SDC\ESB cell showed a substantial increase in $\Phi_{o c}$ and $t_{i o n}{ }_{i o n}$, compared to the single layered, SDC-based SOFC cell. Also, the $\Phi_{o c}$ of the SDCIESB cell was greater than that of SDC at any temperature. This enhancement in _oc and t'ion, is due to the ability of the ESB layer to block electronic conduction. Thus further confirming our bilayer concept. 


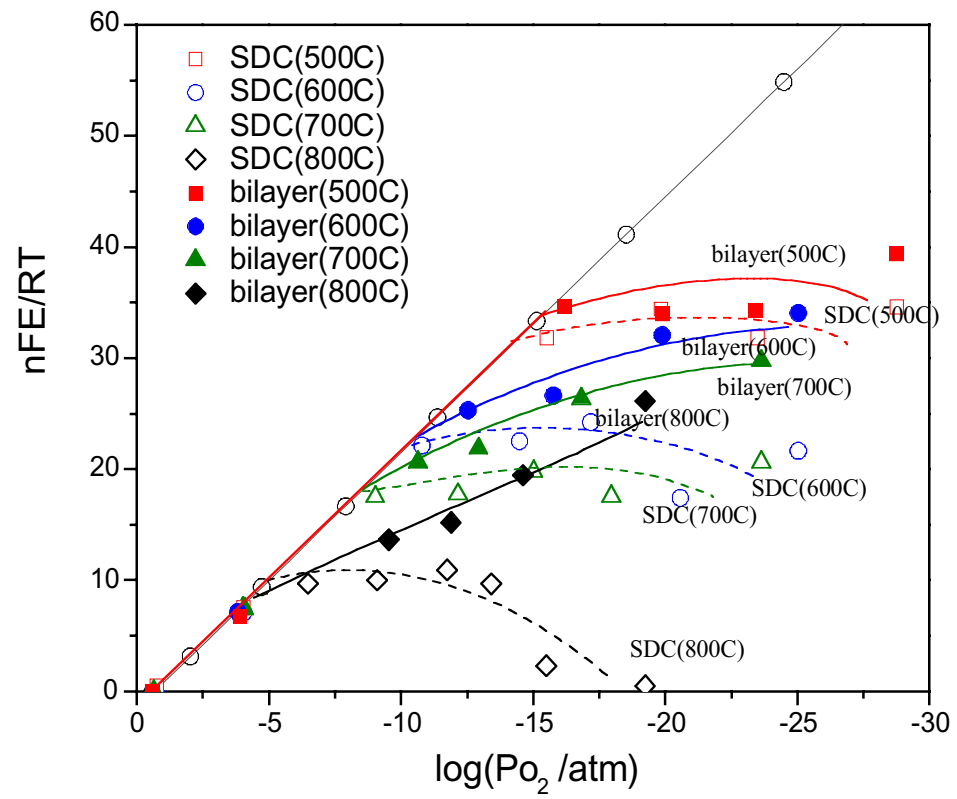

(a)

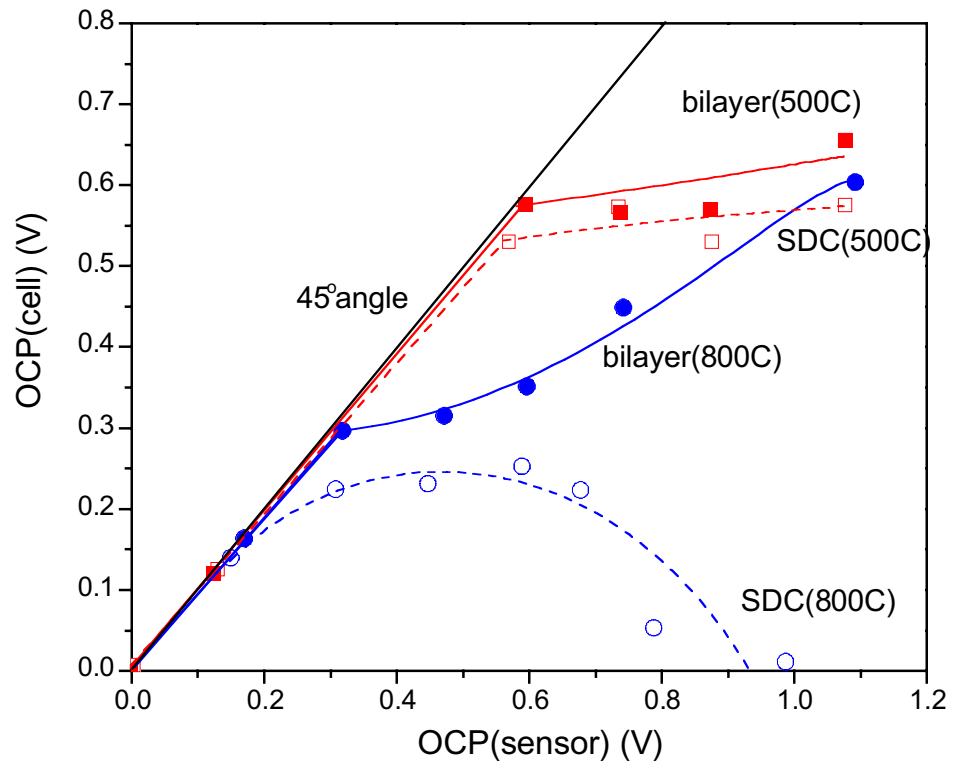

(b)

Figure 6-1. Comparison of $\Phi_{o c}(n F E / R T)$ of fuel cells in Eqs. (4.1) and (6-1) as a function of (a) $P_{\mathrm{O}_{2}}$ and (b) sensor voltage $\left(\Phi_{t h}\right)$; ESB thickness: $1.3 \mu \mathrm{m}$. 


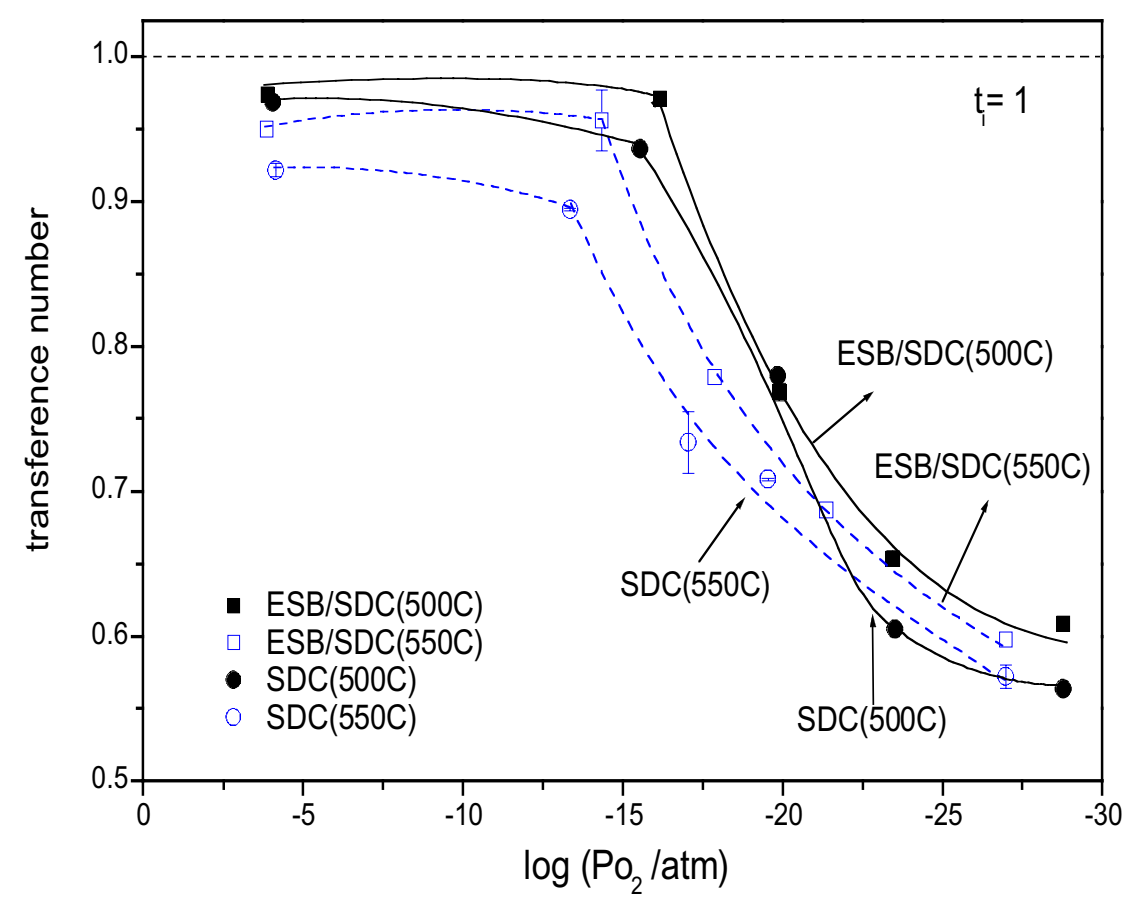

(a)

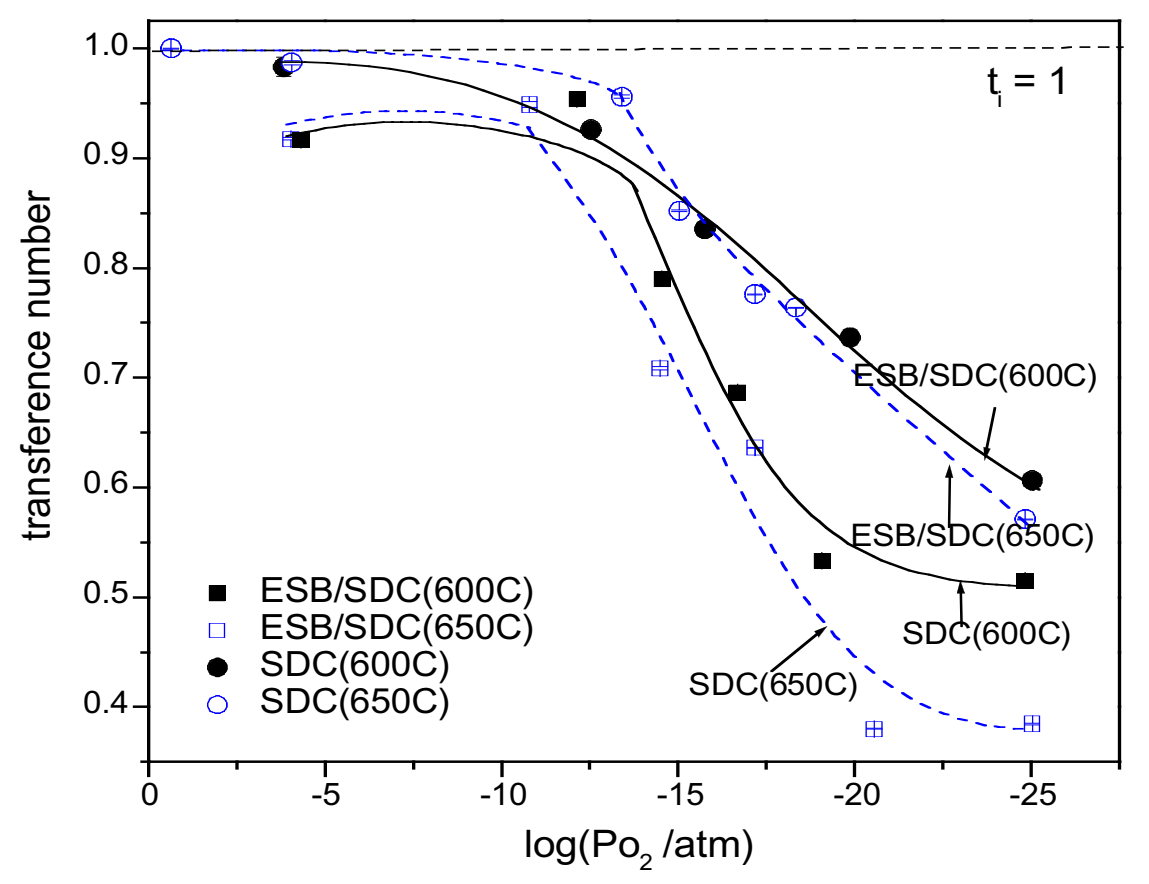

(b) 


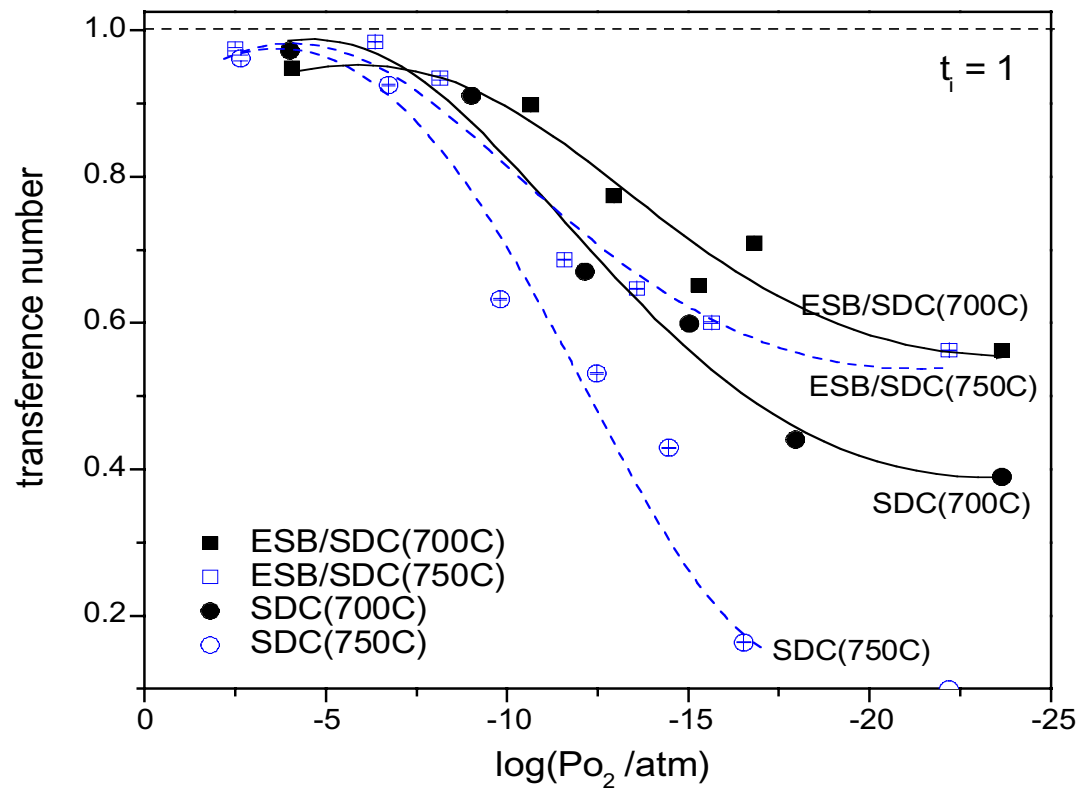

(c)

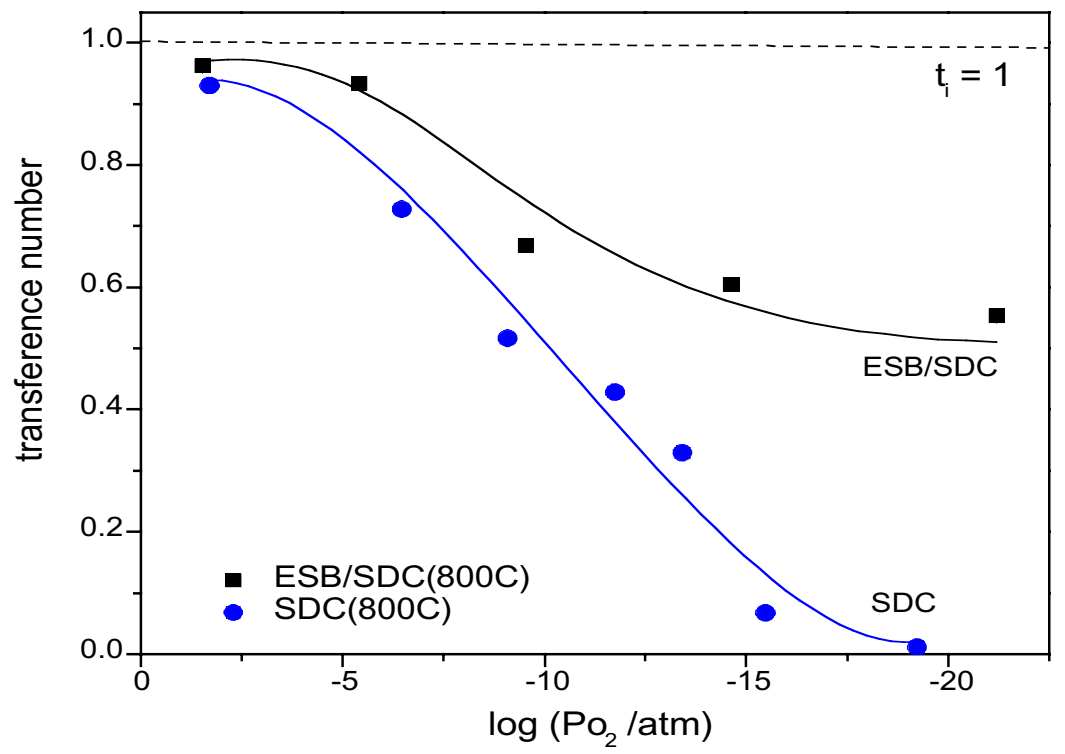

(d)

Figure 6-2. Comparison of $t_{i o n}$ of fuel cells in Eqs. (4.1) and (6-1) as a function of $P_{\mathrm{O}_{2}}$ at (a) 500 and 550rc, (b) 600 and 650rC, (c) 700 and 750rC, and (d) 800rC. 


\subsection{EFFECT OF RELATIVE THICKNESS ON $\Phi_{o c}$ AND $t^{\prime}{ }_{i o n}$}

To investigate the effect of relative thickness of bilayered SDCLESB electrolytes on $\Phi_{o c}$ and $t_{i o n}^{\prime}$, two cells were prepared by depositing ESB layers via PLD on identical SDC substrates. The thicknesses of the ESB layers $(0.8 \mu \mathrm{m}$ and $1.3 \mu \mathrm{m})$ were determined from profilometry and SEM analysis. Henceforth, each bilayer substrate is identified as SDC\ESB0.8 and SDC\ESB 1.3 corresponding to ESB thicknesses of $0.8 \mu \mathrm{m}$ and $1.3 \mu \mathrm{m}$ respectively. Table 6-1 shows the relative thickness of SDCIESB electrolytes, including some of our previous data [59].

Open circuit voltage and $t_{i o n}^{\prime}$ of SDC LESB0.8 and SDCLESB 1.3 cells are shown in Figures 6-3 and 6-4 respectively, as a function of $P_{\mathrm{O}_{2}}$ for $500{ }^{\circ} \mathrm{C} \leq T \leq 800{ }^{\circ} \mathrm{C}$. Table 6-2 shows the values of $P_{\mathrm{O}_{2}}$ at which $t^{\prime}{ }_{i o n}$ falls below 0.9 for SDC and SDCLESB. Open circuit voltage and $t_{i o n}^{\prime}$ of SDC\ESB0.8 and SDC\ESB1.3 cells show similar results, because the relative thickness of SDCIESB layers in the two cells is of the same order of magnitude.

To further explore the effect of the relative thickness of the constituent layers of SDCIESB bilayers, $\Phi_{o c}$ and $t_{i o n}^{\prime}$ data from Wachsman et al. [59] - taken for SDC\ESB cells under identical conditions of $\left(\mathrm{H}_{2} / \mathrm{H}_{2} \mathrm{O}\right.$ on the anode side and air on the cathode side) - was included in the analyses. As shown in Figures 6-5 and 6-6, in the temperature range studied, $\Phi_{o c}$ and $t^{\prime}{ }_{i o n}$ increased significantly as the ESB film thickness increased, approaching $\sim 1 \mathrm{~V}$ and $\sim 0.9$ respectively for a relative thickness (ESBISDC) of 0.03 . Therefore, the theoretical (Nernst) voltage and a $t_{i o n}^{\prime}$ of unity is achievable with a thicker ESB layer relative to SDC (for a given total thickness). This again confirms the bilayer concept.

Table 6-1. Relative Thicknesses of SDCIESB Electrolytes

\begin{tabular}{cccc}
\hline Electrolytes & $\begin{array}{c}\text { SDC layer thickness } \\
(\mathrm{mm})\end{array}$ & $\begin{array}{c}\text { ESB film thickness } \\
(\mu \mathrm{m})\end{array}$ & $\begin{array}{c}\text { Relative thickness } \\
(\mathrm{ESB} \backslash \mathrm{SDC})\end{array}$ \\
\hline SDCIESB0.8 & 1.601 & 0.8 & $5.0 \leftrightarrow 10^{-4}$ \\
SDCIESB1.3 & 1.756 & 1.3 & $7.4 \leftrightarrow 10^{-4}$ \\
SDCIESB30 $^{\dagger}$ & 1.0 & 30 & $3.0 \leftrightarrow 10^{-2}$ \\
\hline
\end{tabular}

$\dagger$ : relative thickness from previous results (from Wachsman et al. [59]) 


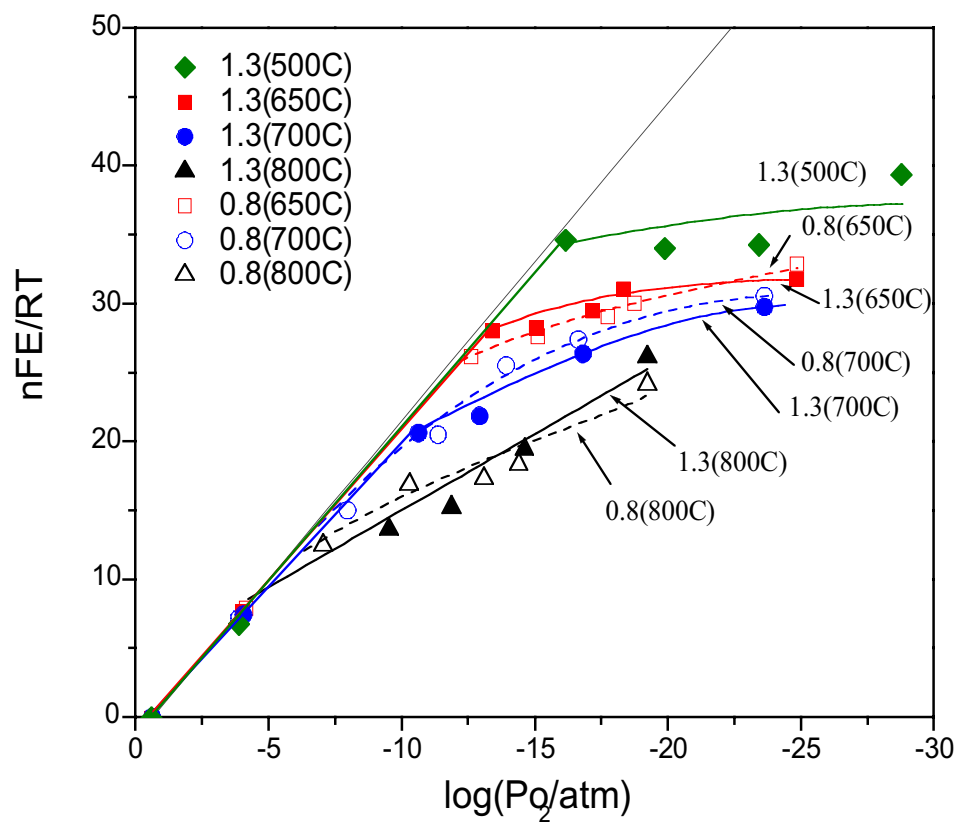

(a)

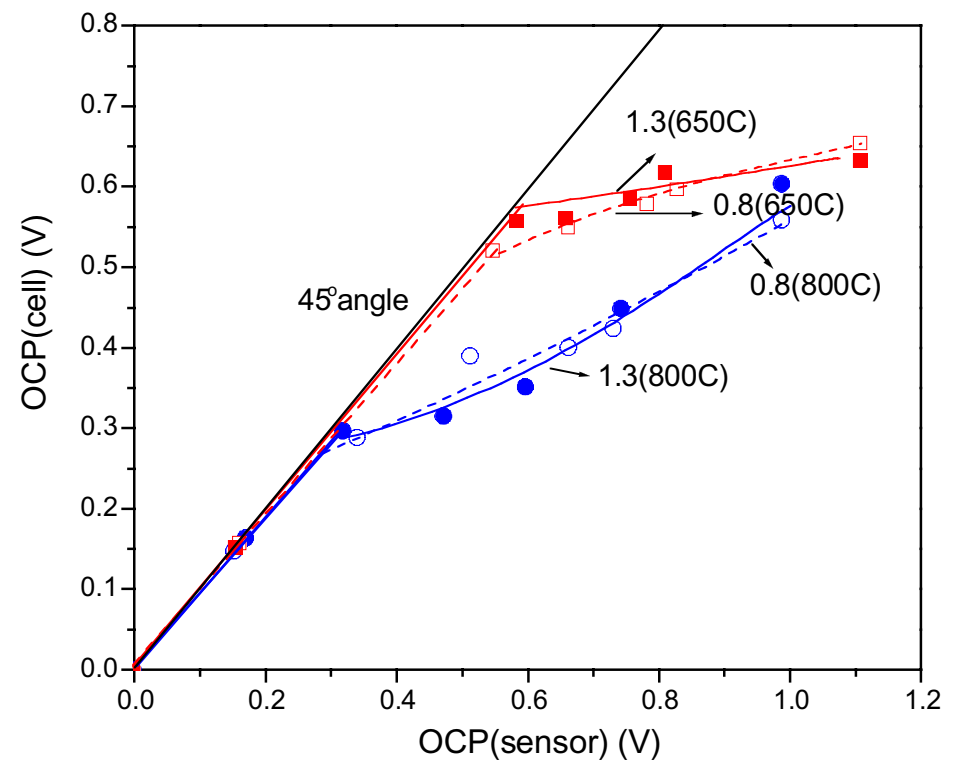

(b)

Figure 6-3. Comparison of $\Phi_{o c}(n F E / R T)$ of SDCLESB0.8 and SDCLESB 1.3 concentration cells (Eq. (6.1)) as a function of (a) $P_{\mathrm{O}_{2}}$ and (b) sensor voltage $\left(\Phi_{t h}\right)$. 


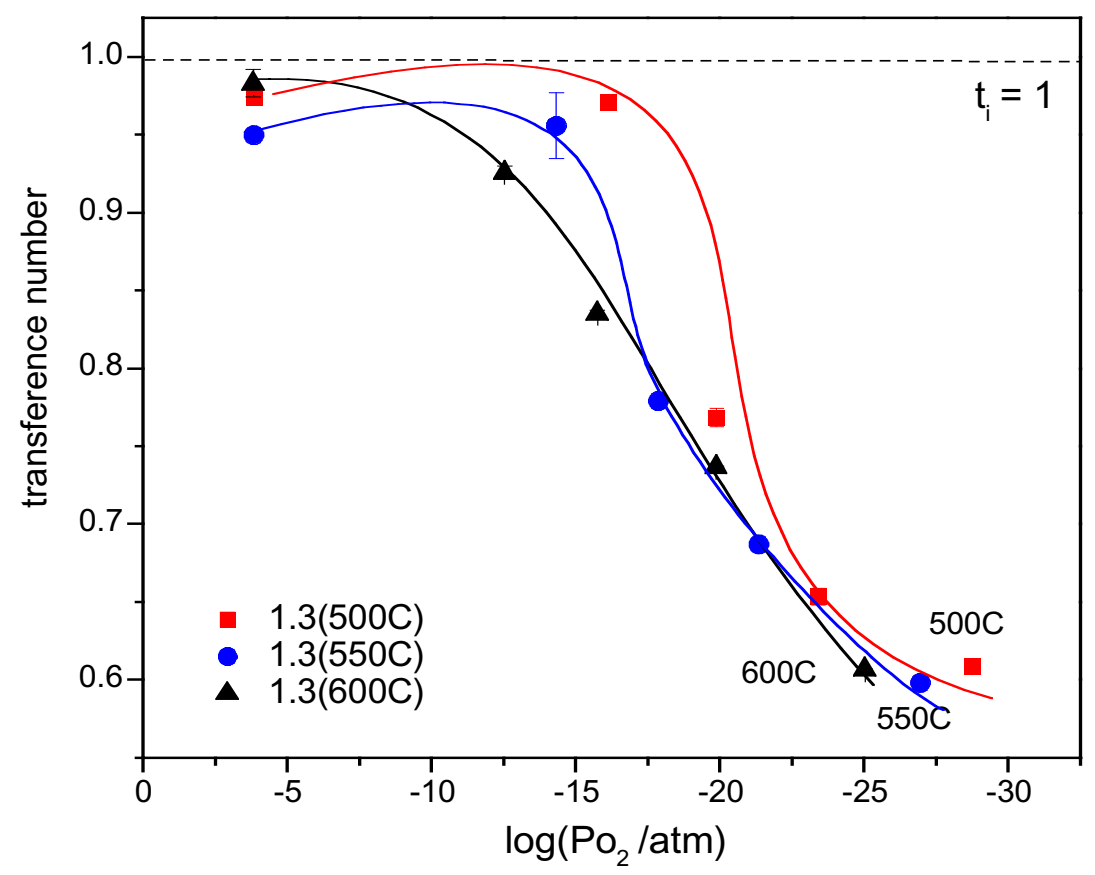

(a)

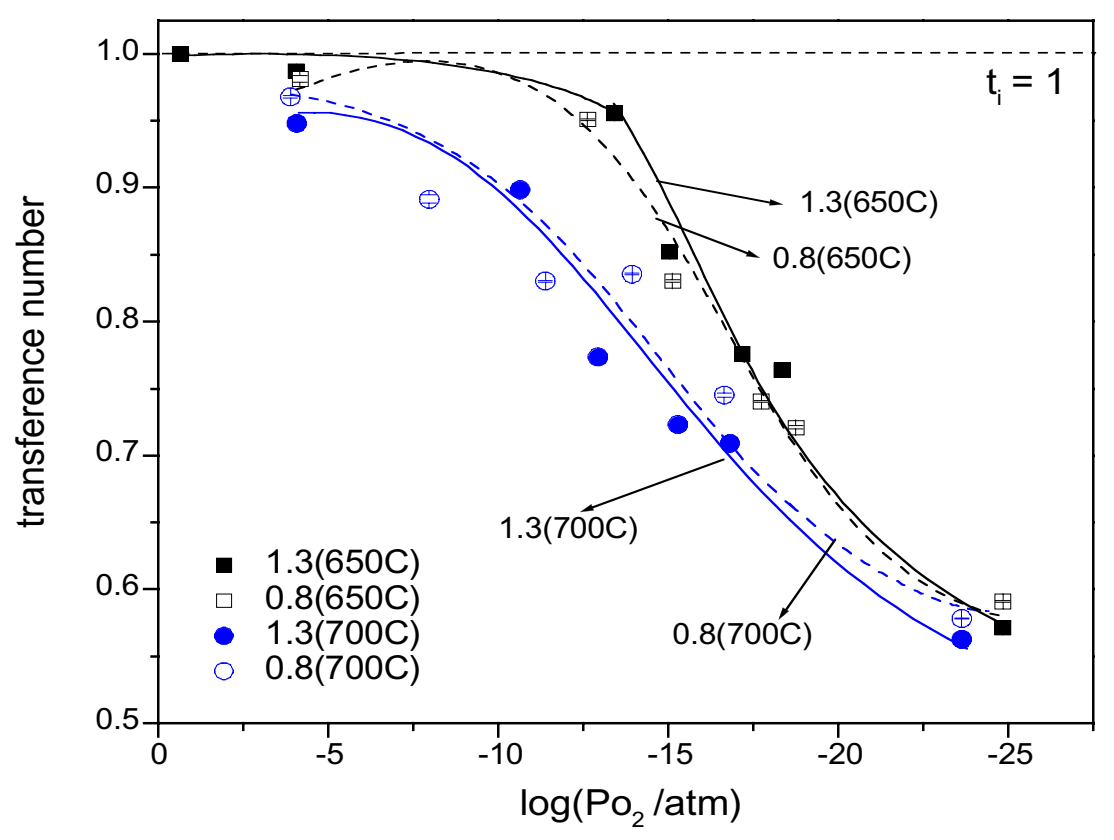

(b) 


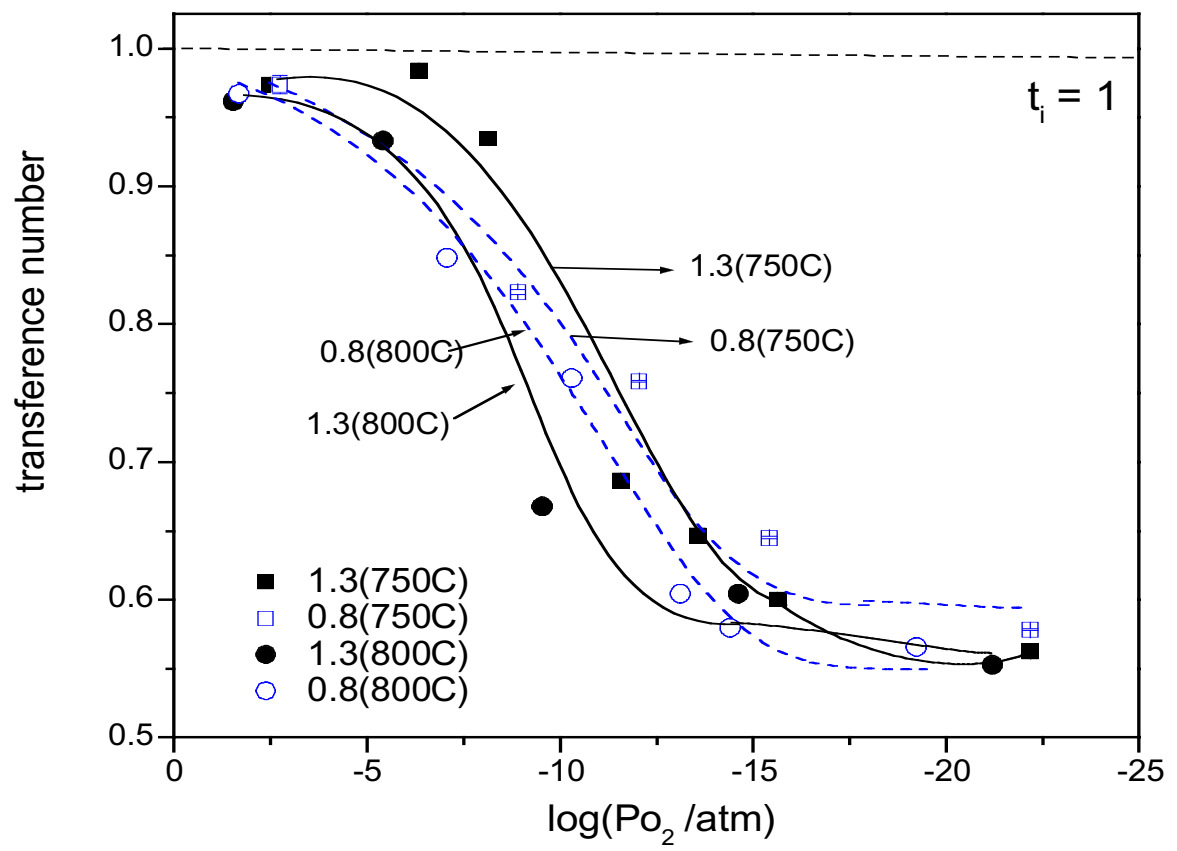

(c)

Figure 6-4. Comparison of $t^{\prime}{ }_{i o n}$ for SDC $\ E S B 0.8$ and SDC\ESB1.3 cells (Eq. (6.1)) as a function of $P_{\mathrm{O}_{2}}$ at (a) 500 and $550{ }^{\circ} \mathrm{C}$, (b) 650 and $700{ }^{\circ} \mathrm{C}$, (c) 750 and $800{ }^{\circ} \mathrm{C}$.

Table 6-2. Oxygen Partial Pressure at which $t_{i o n}^{\prime}=0.90$ for SDC, SDCIESB0.8 and SDCIESB 1.3 cells (in Eqs. (4.1) and (6-1)).

\begin{tabular}{cccc}
\hline Temperatures $\left({ }^{\circ} \mathrm{C}\right)$ & $\begin{array}{c}\text { SDC } \\
(0)^{1}\end{array}$ & $\begin{array}{c}0.8 \text { SDClESB } \\
\left(5.0 \times 10^{-4}\right)^{1}\end{array}$ & $\begin{array}{c}1.3 \text { SDClESB } \\
\left(7.4 \times 10^{-4}\right)^{1}\end{array}$ \\
\hline 500 & $\sim 10^{-17.5}$ & & $\sim 10^{-17.5}$ \\
550 & $\sim 10^{-14}$ & & $\sim 10^{-16}$ \\
600 & $\sim 10^{-13.5}$ & & $\sim 10^{-15}$ \\
650 & $\sim 10^{-13}$ & $\sim 10^{-14.5}$ & $\sim 10^{-14.5}$ \\
700 & $\sim 10^{-9.5}$ & $\sim 10^{-9}$ & $\sim 10^{-11}$ \\
750 & $\sim 10^{-7.5}$ & $\sim 10^{-8}$ & $\sim 10^{-8.5}$ \\
800 & $\sim 10^{-4}$ & $\sim 10^{-7}$ & $\sim 10^{-7}$ \\
\hline
\end{tabular}

()$^{1}$ : relative thickness of electrolytes (SDClESB) 


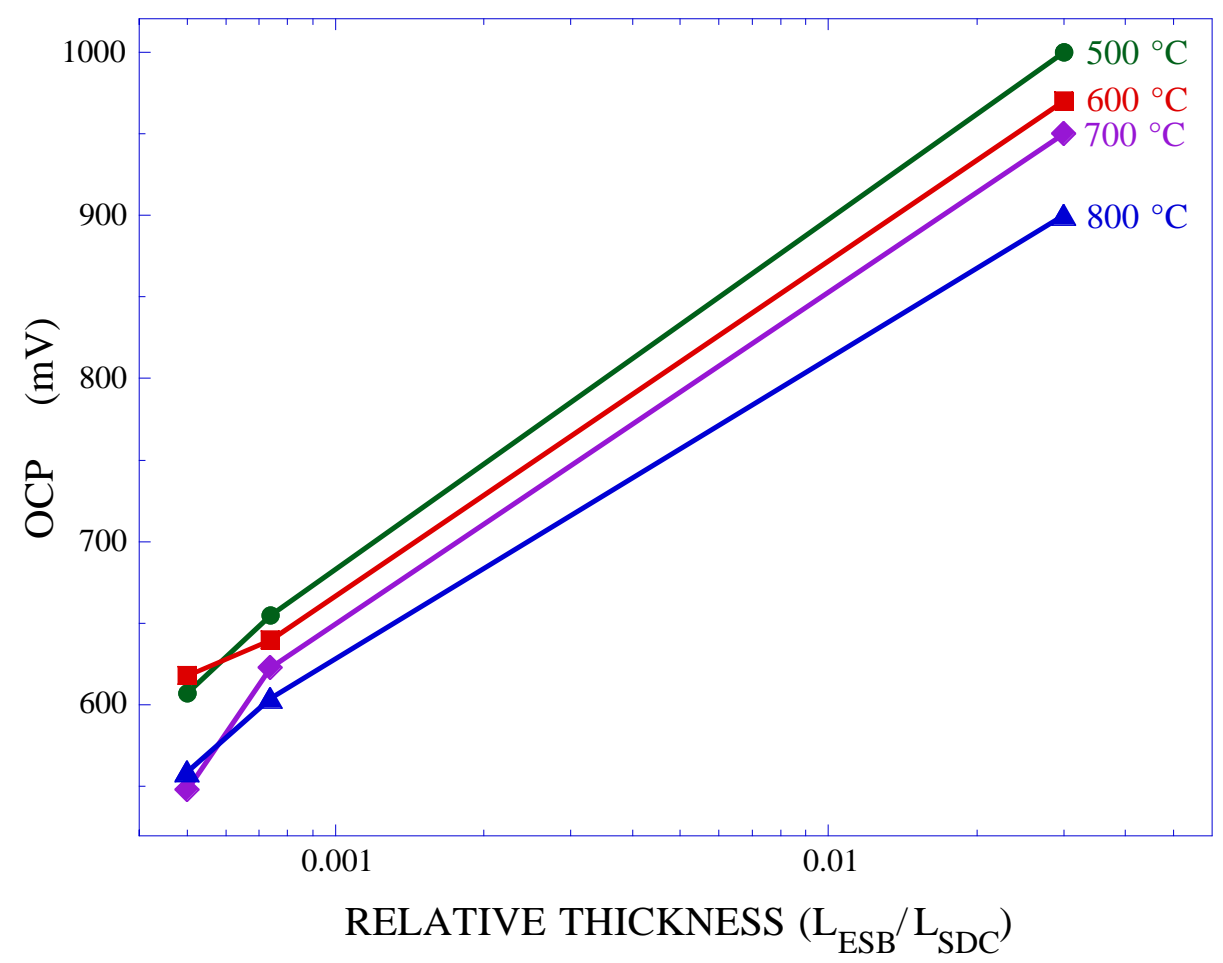

Figure 6-5. Dependence of $\Phi_{o c}$ on relative thickness of the constituent layers of SDClESB SOFC.

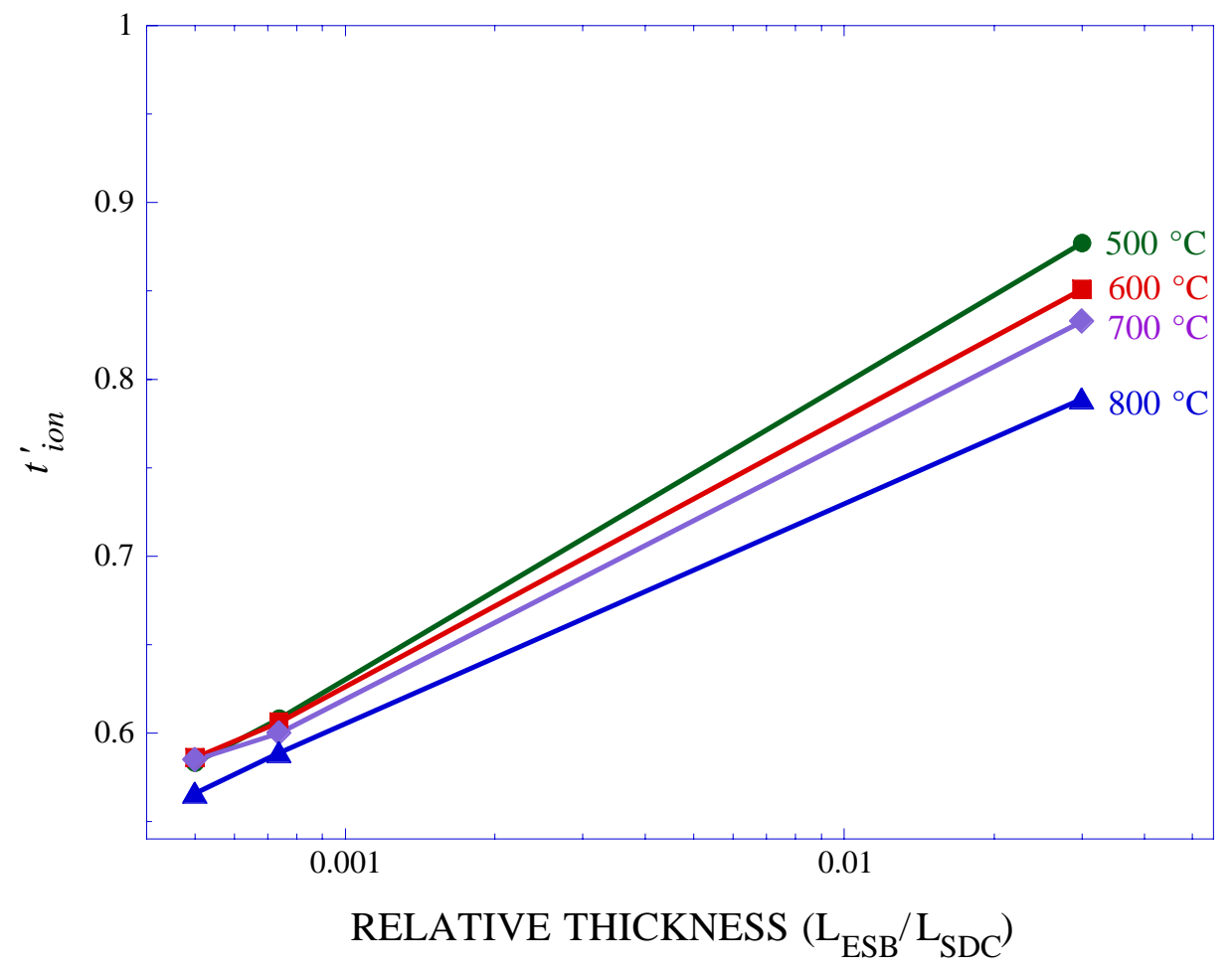

Figure 6-6. Dependence of $t_{i o n}^{\prime}$ on relative thickness of the constituent layers of SDCLESB SOFC. 


\section{REFERENCES}

1. K. Mehta, S. J. Hong, J. -F. Jue and A. V. Virkar, in Solid Oxide Fuel Cells III, S. C. Singhal and H. Iwahara, ed., PV 93-4, p. 92, Electrochem. Soc. Proc. Series.

2. P. Soral, U. Pal and W. Worrell, J. Electrochem. Soc. 145 (1998) 99.

3. P. Shuk, H. -D. Wiemhöfer, U. Guth, W. Gopel and M. Greenblatt, Solid State Ionics 89 (1996) 179.

4. A. M. Azad, S. Larose and S. A. Akbar, J. Mat. Sci. 29 (1994) 4135.

5. J. Van herle, T. Horita, T. Kawada, N. Sakai, H. Yokoko and M. Dokiya, Solid State Ionics, 86-88 (1996) 1255.

6. W. Huang, P. Shuk, and M. Greenblatt, Solid State Ionics, 100 (1997) 23.

7. M. N. Rahaman, Ceramic Processing and Sintering, Marcel Decker, Inc., New York 1995.

8. P. D. Battle, C. R. A. Catlow, J. W. Heap and L. M. Moroney, J. Solid State Chem. 63, 8 (1986)

9. N. Jiang and E.D. Wachsman, J. Am. Ceram. Soc., 82 [11] 3057 - 64 (1999)

10. E. D. Wachsman, S. Boyapati, M. J. Kaufman, N. Jiang, J. Am. Ceram. Soc. 83 (2000) 1964 $-1968$

11. E. D. Wachsman, N. Jiang, D. M. Mason and D. A. Stevenson, Proc. Electrochem., Soc., 89 $-11,15(1989)$

12. S. Boyapati, E. D. Wachsman and B. C. Chakoumakos, Solid State Ionics 138 (2001) 293304

13. S. Boyapati, E. D. Wachsman and N. Jiang, Solid State Ionics, 140 (2001) 149-160

14. E. D. Wachsman, S. Boyapati and N. Jiang, Ionics, 7 (2001) p. 1

15. T. Takahashi and H. Iwahara, J. of Appl. Electrochem. 3 (1973) 65-72

16. M. J. Verkerk and A. J. Burggraaf, J. Electrochem., 128 (1981) 75

17. G. Meng, C. Chen, X. Han, P. Yang and D. Peng, J. Mat. Sci. 28-30 (1988) 533 
18. K. Z. Fung and A. V. Virkar, J. Am. Ceram. Soc., 74 [8] 1970-80 (1991)

19. B.C.H. Steele, Solid State Ionics, 129 (2000) 95.

20. A. V. Virkar, G. R. Miller, and R. S. Gordon, J. Am. Ceram. Soc., 61 (1978) 250.

21. S. J. Hong, K. Mehta, and A.V. Virkar, J. Electrochem. Soc., 145 (1998) 638.

22. C. Tian and S. Chan, Solid State Ionics, 134 (2000) 89.

23. R. Gerhardt and A. S. Nowick, J. Am. Ceram. Soc., 69 (1986) 641.

24. R. Gerhardt, A. S. Nowick, M. E. Mochel, and I. Dumler, J. Am. Ceram. Soc., 69 (1986) 647.

25. E. Schouler, G. Girond, M. Kleitz, J. Chem. Phys., 70 (1973).

26. G. M. Christie and F. P. F. van Berkel, Solid State Ionics, 83 (1996) 17.

27. P. L Chen and I. W. Chen, J. Am. Ceram. Soc., 79 (1996) 1793.

28. S. Jung, E. D. Wachsman and N. Jiang, submitted, Ionics

29. E.D. Wachsman, N. Jiang, D. M. Mason, and D. A. Stevenson, Proceedings of the first international syposium on Solid Oxide Fuel Cells 89-11, ed. S. C. Singhal Electrochem. Soc. Proc. Series.

30. E. D. Wachsman, S. Boyapati, M. J. Kaufman, and N. Jiang, Solid State Ionic Devices: Proc. Electrochem. Soc., 42 (1999).

31. D. Y. Wang, D. S. Park, J. Griffith, and A. S. Nowick, Solid State Ionics, 2 (1981) 95.

32. S. Ohara, R. Maric, X. Zhang, K. Mukai, T. Fukui, H. Yoshida, T. Inagaki, K. Miura, J. Power Sources, 86, 455 (2000).

33. J. Will, A. Mitterdorfer, C. Kleinlogel, D. Perednis, L.J. Gaukler, Solid State Ionics, 131, 79 (2000).

34. S. deSouza, S.J. Visco, L.C. DeJonghe, J. Electrochem. Soc., 144, L35 (1997).

35. K.T. Miller, F.F. Lange, D.B. Marshall, J. Mater. Res., 5, 151 (1990).

36. K. Duncan, Ph. D. Thesis, University of Florida (2001).

37. J. Newman, Electrochemical Systems (Prentice-Hall, 1991). 
38. F. W. Poulsen, Solid State Ionics 129 (2000) 145.

39. F. W. Poulsen, J. Solid State Chem. 143 (1999) 115.

40. I. Riess and J. Schoonman in: Handbook of Solid State Electrochemistry, ed. P. J. Gellings and H. J. M. Bouwmeester (CRC Press, 1997) chap. 8, p. 269.

41. W. Weppner in: Handbook of Solid State Electrochemistry, ed. P. J. Gellings and H. J. M. Bouwmeester (CRC Press, 1997) chap. 9, p. 295.

42. S. Wang, H. Inaba, H. Tagawa, M. Dokiya and T. Hashimoto, Solid State Ionics 107 (1998) 73.

43. T. Kobayashi, S. Wang, M. Dokiya, Hiroaki T. and T. Hashimoto, Solid State Ionics 126 (1999) 349.

44. H. Inaba and H. Tagawa, Solid State Ionics 83 (1996) 1.

45. M. Godickemeier, K. Sasaki, L. Gauckler and I. Riess, J. Electrochem. Soc. 144 (1997) 1635.

46. M. Godickemeier and L. Gauckler, J. Electrochem. Soc. 145 (1998) 414.

47. K. Eguchi, T. Setoguchi, T. Inoue and H. Arai, Solid State Ionics 52 (1992) 265.

48. J. -Y. Park, M. Sc. Thesis, University of Florida, (2001).

49. M. R. Spiegel in, Schaum's Outline Series: Theory and Problems of Statistics 2nd. edition (McGraw-Hill, 1994).

50. P. J. Gellings, H. J. A. Koopmans and A. J. Burggraaf, App. Catalysis 39 (1988) 1

51. E. D. Wachsman, Ph. D. Thesis, Stanford University (1990).

52. K. L. Duncan and E. D. Wachsman, Ionics 6 (2000) 145.

53. C. Wagner, Z. Elektrochem. 60 (1956).4.

54. E. D. Wachsman and K. L. Duncan, Proceedings of the International Symposium on Solid State Ionic Devices, Vol. 99-13 (The Electrochemical Society, Inc. 1999), p. 159.

55. T. Takahashi, H. Iwahara and T. Arao, J. App. Electrochem. 7 (1977) 299.

56. M. J. Verkerk, K. Keizer and A. J. Burggraaf, J. App. Electrochem. 10 (1980) 81. 
57. M. J. Verkerk and A. J. Burggraaf, J. App. Electrochem. 10 (1980) 677.

58. V. V. Kharton and F. M. B. Marques, Solid State Ionics 140 (2001) 381.

59 E. D. Wachsman, P. Jayaweera, N. Jiang, D.M. Lowe, and B.G. Pound, J. Electrochem. Soc., 144 (1997) 233. 